\title{
MOJAVE: MONITORING OF JETS IN ACTIVE GALACTIC NUCLEI WITH VLBA EXPERIMENTS. VIII. FARADAY ROTATION IN PARSEC-SCALE AGN JETS
}

\author{
Talvikki Hovatta ${ }^{1,2}$, Matthew L. Lister ${ }^{1}$, Margo F. Aller ${ }^{3}$, Hugh D. Aller ${ }^{3}$, Daniel C. Homan ${ }^{4}$, \\ Yuri Y. Kovalev ${ }^{5,6}$, Alexander B. PushKarev ${ }^{6,7,8}$, and Tuomas Savolainen ${ }^{6}$ \\ ${ }^{1}$ Department of Physics, Purdue University, 525 Northwestern Ave. West Lafayette, IN 47907, USA \\ ${ }^{2}$ Cahill Center for Astronomy \& Astrophysics, California Institute of Technology, 1200 E. California Blvd, Pasadena, CA 91125, USA; thovatta@ caltech.edu \\ ${ }^{3}$ Department of Astronomy, University of Michigan, 817 Dennison Building, Ann Arbor, MI 48109-1042, USA \\ ${ }^{4}$ Department of Physics and Astronomy, Denison University, Granville, OH 43023, USA \\ ${ }^{5}$ Astro Space Center of Lebedev Physical Institute, Profsoyuznaya 84/32, 117997 Moscow, Russia \\ ${ }^{6}$ Max-Planck-Institut für Radioastronomie, Auf dem Hügel 69, 53121 Bonn, Germany \\ ${ }^{7}$ Pulkovo Observatory, Pulkovskoe Chaussee 65/1, 196140 St. Petersburg, Russia \\ ${ }^{8}$ Crimean Astrophysical Observatory, 98409 Nauchny, Crimea, Ukraine \\ Received 2012 January 11; accepted 2012 May 25; published 2012 September 11
}

\begin{abstract}
We report observations of Faraday rotation measures for a sample of 191 extragalactic radio jets observed within the MOJAVE program. Multifrequency Very Long Baseline Array observations were carried out over 12 epochs in 2006 at four frequencies between 8 and $15 \mathrm{GHz}$. We detect parsec-scale Faraday rotation measures in 149 sources and find the quasars to have larger rotation measures on average than BL Lac objects. The median core rotation measures are significantly higher than in the jet components. This is especially true for quasars where we detect a significant negative correlation between the magnitude of the rotation measure and the de-projected distance from the core. We perform detailed simulations of the observational errors of total intensity, polarization, and Faraday rotation, and concentrate on the errors of transverse Faraday rotation measure gradients in unresolved jets. Our simulations show that the finite image restoring beam size has a significant effect on the observed rotation measure gradients, and spurious gradients can occur due to noise in the data if the jet is less than two beams wide in polarization. We detect significant transverse rotation measure gradients in four sources $(0923+392,1226+023$, $2230+114$, and 2251+158). In 1226+023 the rotation measure is for the first time seen to change sign from positive to negative over the transverse cuts, which supports the presence of a helical magnetic field in the jet. In this source we also detect variations in the jet rotation measure over a timescale of three months, which are difficult to explain with external Faraday screens and suggest internal Faraday rotation. By comparing fractional polarization changes in jet components between the four frequency bands to depolarization models, we find that an external purely random Faraday screen viewed through only a few lines of sight can explain most of our polarization observations, but in some sources, such as $1226+023$ and $2251+158$, internal Faraday rotation is needed.
\end{abstract}

Key words: BL Lacertae objects: general - galaxies: active - galaxies: jets - polarization - quasars: general radio continuum: galaxies

Online-only material: color figures, extended figure, figure set, machine-readable and VO tables

\section{INTRODUCTION}

Polarimetric observations of active galactic nucleus (AGN) jets enable studies of the magnetic field structure in outflows. If the jets are launched from the rotating black hole or accretion disk, it is natural to expect that the magnetic field structure in the jets is helical (e.g., Blandford \& Znajek 1977; Meier et al. 2001; Vlahakis \& Königl 2004; McKinney \& Narayan 2007). On the other hand, it is not known whether the helical structure persists parsecs down from the central engine or if it becomes tangled due to re-collimation shocks or interaction with an external medium (e.g., Marscher et al. 2008). Very long baseline interferometry (VLBI) can be used to study the electric vector orientation in the parsec-scale jets of AGNs. In the optically thin part of the jet, the magnetic field orientation is perpendicular to the electric vector position angles (EVPAs). Thus, observations of EVPAs parallel to the jet's direction have resulted in claims of toroidally dominated magnetic fields (e.g., Gabuzda et al. 2004). One should note that relativistic effects make the situation more complicated and when viewed at small angles a toroidally dominated magnetic field can appear poloidal in the observer's frame (Lyutikov et al. 2005). Alternatively the observed magnetic field orientation can be accounted for by shocks compressing the magnetic field perpendicular to the jet (Laing 1980; Hughes et al. 1989).

Polarized waves are affected by Faraday rotation when propagating through non-relativistic plasma within or external to the source (e.g., Burn 1966). This effect can both diminish the observed degree of polarization and rotate the intrinsic EVPAs so that in order to study the intrinsic magnetic field orientation in the jets, the effect must be removed. In the case of external rotation, the effect can be described by a linear dependence between the observed EVPA $\left(\chi_{\text {obs }}\right)$ and wavelength squared $\left(\lambda^{2}\right)$ by

$$
\chi_{\mathrm{obs}}=\chi_{0}+\frac{e^{3} \lambda^{2}}{8 \pi^{2} \epsilon_{0} m^{2} c^{3}} \int n_{e} \mathbf{B} \cdot \mathrm{d} \mathbf{l}=\chi_{0}+\mathrm{RM} \lambda^{2},
$$

where $\chi_{0}$ is the intrinsic EVPA and RM is the rotation measure, related to the electron density $n_{e}$ and the magnetic field component $\mathbf{B}$ parallel to the line of sight. The constant in the equation consists of the charge of the electron $e$, vacuum permittivity $\epsilon_{0}$, mass of the electron $m$, and speed of light $c$. The RM can thus be estimated by observing the EVPA at several frequencies. 
If the Faraday rotation is internal to the jet it means that either the thermal plasma causing the rotation is intermixed with the emitting plasma, or the relativistic particle spectrum extends to low energies. For total rotations larger than $45^{\circ}$ internal Faraday rotation is expected to cause severe depolarization (Burn 1966), which is not often seen (Zavala \& Taylor 2004), but for smaller total rotations the internal rotation can appear linear and follow Equation (1). External Faraday rotation could be caused by a screen very close to the jet itself where it can also interact with the jet (e.g., a sheath) or by a more distant screen such as the broad- or narrow-line regions, or even intergalactic and Galactic plasma. Distinguishing between the different alternatives can be very difficult, especially in the case of small rotations if additional information is not available (e.g., Homan et al. 2009).

Over the past few decades there have been numerous studies of Faraday rotation in parsec-scale jets associated with active galaxies. One of the largest is by Taylor $(1998,2000)$ and Zavala \& Taylor $(2002,2003$, 2004) who report Faraday rotation measures (RMs) for a sample of 40 AGNs. They find the typical absolute core RMs to be in a range of 500 to several thousand $\mathrm{rad} \mathrm{m}^{-2}$ in the observer's frame. Additionally, they report variability in several RMs over a time span of months to years, ruling out the narrow-line region as the origin for the Faraday rotation. Instead they suggest that the Faraday rotation is caused by a screen close to the jet. Similar conclusions about the screen were drawn by Asada et al. (2002) who detected a transverse rotation measure gradient in the jet of 3 C 273. They interpreted the gradient as a signature of a helical magnetic field in the sheath surrounding the jet. Several other claims of transverse gradients have been published (e.g., Gabuzda et al. 2004; Asada et al. 2008b; Gómez et al. 2008; Mahmud et al. 2009; Croke et al. 2010) but due to some uncertainties regarding transversely unresolved jets the issue remains controversial (Taylor \& Zavala 2010).

Due to the complex nature of the sources and their surroundings, the situation is often more complicated. In addition to a helical field, interactions with the surrounding intergalactic medium will cause distinct RM structures (Gómez et al. 2008). Additionally, beam effects can severely complicate the interpretation of the maps as shown by simulations (Broderick \& McKinney 2010). Starting from general relativistic magnetohydrodynamical simulations, Broderick \& McKinney (2010) created a canonical jet model and calculated RM maps, which they convolved with different image restoring beam sizes to create unresolved and resolved jets. They showed that within one beam width from the optically thick core, any gradient seen in the RM map is generally unreliable, and only at a resolution obtained with $43 \mathrm{GHz}$ Very Long Baseline Array (VLBA) observations are gradients in agreement with expected values, although these are still suppressed in magnitude. In the case of an optically thin jet, it could be possible to detect a gradient if the jet is surrounded by a helical field even if the jet is unresolved (above $8 \mathrm{GHz}$ VLBA resolution), but the magnitude of the gradient may be suppressed due to the beam effects.

In this paper we study the statistical properties of Faraday rotation in AGNs by using a large sample of objects that are part of the MOJAVE (Monitoring of Jets in Active Galactic Nuclei with VLBA Experiments) survey (Lister et al. 2009a). Our goal was to create a set of RM maps in which all potential sources of error in the data processing have been accounted for. Therefore, we have performed extensive simulations of the errors in polarization and Faraday rotation maps and have assessed when an RM gradient can be called significant. These simulations show that the finite beam size of VLBI observations has a large effect on the observed Faraday rotation, and caution needs to be taken when interpreting the maps. Neighboring pixels in beam-convolved images are not independent for a typical VLBI pixel size and the RM maps generally consist only of a few independent measurements.

We describe our observations and the detailed data analysis process in Section 2. The results of our statistical study are reported in Section 3. We discuss our results in light of depolarization models, observed RM gradients, and time variability in Section 4. Our conclusions are summarized in Section 5. In Appendix A we discuss the effects of relative image alignment on RM maps, and in Appendices B and C we discuss observational errors in polarization and $\mathrm{RM}$ images. Throughout the paper we use a cosmology where $H_{0}=71 \mathrm{~km} \mathrm{~s}^{-1} \mathrm{Mpc}^{-1}$, $\Omega_{M}=0.3$, and $\Omega_{\Lambda}=0.7$ (e.g., Komatsu et al. 2009).

\section{OBSERVATIONS AND DATA REDUCTION}

Our sample consists of 191 AGNs observed within the MOJAVE VLBA survey (Lister et al. 2009a). It includes 134 sources of the complete flux density-limited MOJAVE-1 sample, for which we monitor total intensity and polarization changes of 135 AGN jets above declination $-20^{\circ}$, which have exceeded $15 \mathrm{GHz}$ flux density $1.5 \mathrm{Jy}\left(2 \mathrm{Jy}\right.$ at $\delta<0^{\circ}$ ) at any epoch between 1994 and 2002. The rest of the sources belong to the MOJAVE-2 sample, ${ }^{9}$ which includes sources from the $2 \mathrm{~cm}$ survey (Kellermann et al. 2004), gamma-ray blazars, and other sources with unusual jet properties.

The sources were observed with VLBA in 2006 over 12 epochs with about monthly separation, each epoch containing 18 sources (except for epoch 2006 February 12, which included only 14 sources and epoch 2006 April 28, which included 17 sources). The observations were made in dual polarization mode using frequencies centered at 8.104, 8.424 ( $X$ band), 12.119, and $15.369 \mathrm{GHz}$ ( $U$ band). This setup was chosen because VLBA observes the gain centered at $8.4 \mathrm{GHz}$, and $8.1 \mathrm{GHz}$ was chosen as the low frequency end of the $X$ band. The bandwidths were 16 and $32 \mathrm{MHz}$ for the $X$ and $U$ bands, respectively. The observations were recorded with a bit rate of $128 \mathrm{Mbits}^{-1}$. In the $X$ bands the observations consist of two intermediate frequencies (IFs) in both frequencies and in the $U$ bands they consist of four IFs. All 10 VLBA antennas were observing except at epoch 2006 August 9 when Pie Town was not included. The sources and their observing epochs are listed in Table 1. A total of 20 sources were observed twice during the year.

\subsection{Data Reduction}

The initial data reduction and calibration were performed following the standard procedures described in the AIPS cookbook. ${ }^{10}$ All the frequency bands were treated separately throughout the data reduction process. The imaging and self-calibration were done in a largely automated way using the Difmap package (Shepherd 1997). For more details see Lister et al. (2009a) for the standard data reduction and imaging process and Lister \& Homan (2005) for the calibration of the polarization data.

All the sources were fitted with circular or elliptical Gaussian components using the standard "modelfit" procedure in the Difmap package. The $15 \mathrm{GHz}$ maps were fitted already as a

\footnotetext{
9 http://www.physics.purdue.edu/astro/MOJAVE/allsources.shtml

10 http://www.aips.nrao.edu
} 
Table 1

Sources and Their Rotation Measure Properties

\begin{tabular}{|c|c|c|c|c|c|c|c|c|c|}
\hline $\begin{array}{l}\text { IAU Name } \\
\text { (1) }\end{array}$ & $\begin{array}{c}\text { Other Name } \\
\text { (2) }\end{array}$ & $\begin{array}{l}z \\
(3)\end{array}$ & $\begin{array}{l}\text { Opt. Cl. } \\
\text { (4) }\end{array}$ & $\begin{array}{l}\beta \text { app } \\
\text { (c) } \\
(5)\end{array}$ & $\begin{array}{c}\text { Epoch } \\
\text { (6) }\end{array}$ & $\begin{array}{c}\text { Gal. RM } \\
\left(\operatorname{rad~m^{-2}}\right) \\
(7)\end{array}$ & $\begin{array}{c}\text { Med. RM } \\
\left(\mathrm{rad} \mathrm{m}^{-2}\right) \\
(8)\end{array}$ & 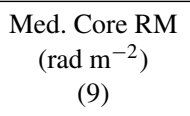 & $\begin{array}{c}\text { Med. Jet RM } \\
\left(\operatorname{rad~m^{-2}}\right) \\
(10)\end{array}$ \\
\hline $0003-066$ & NRAO 005 & 0.3467 & B & 8.4 & 2006 Jul 7 & 4.5 & -20.7 & -34.8 & 130.0 \\
\hline $0003+380$ & S4 0003+38 & 0.229 & Q & $\ldots$ & 2006 Mar 9 & -90.0 & $\ldots$ & $\ldots$ & $\ldots$ \\
\hline $0003+380$ & $\mathrm{~S} 40003+38$ & 0.229 & Q & $\ldots$ & 2006 Dec 1 & -90.0 & 6053.3 & 6053.3 & $\ldots$ \\
\hline $0007+106$ & III Zw 2 & 0.0893 & G & 1.2 & 2006 Jun 15 & -3.4 & 604.2 & 604.2 & $\ldots$ \\
\hline $0010+405$ & $4 \mathrm{C}+40.01$ & 0.256 & Q & $\ldots$ & 2006 Apr 5 & -77.8 & $\ldots$ & $\ldots$ & $\ldots$ \\
\hline $0010+405$ & $4 \mathrm{C}+40.01$ & 0.256 & Q & $\ldots$ & 2006 Dec 1 & -77.8 & $\ldots$ & $\ldots$ & $\ldots$ \\
\hline $0016+731$ & S5 0016+73 & 1.781 & Q & 8.1 & 2006 Aug 9 & -9.1 & 264.9 & 264.9 & $\ldots$ \\
\hline
\end{tabular}

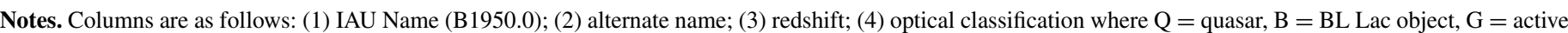

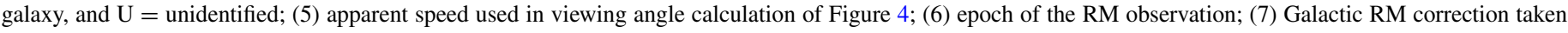
from Taylor et al. (2009); (8) median RM over the source; (9) median RM over the core area; (10) median RM over the jet.

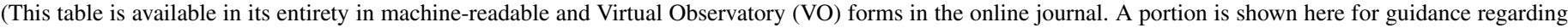
its form and content.)

part of the MOJAVE survey (Lister et al. 2009b). Since one of our goals was to use the optically thin components in the jets to align our images, we used these $15 \mathrm{GHz}$ model fits as a starting point for the other bands and modified the fit if needed.

As the $(u, v)$ plane coverage differs in the bands with higher frequency maps resolving smaller structures, we can get spurious features in the rotation measure maps, especially near the core region where there can be many components blending within the beam at lower frequencies. Therefore, in order to have comparable $(u, v)$ coverage in all the bands, we flagged the long baselines from the 15 and $12 \mathrm{GHz}$ maps and short baselines from the $8 \mathrm{GHz}$ maps. The resulting typical $(u, v)$ range in our data is $7.3-231$ mega- $\lambda$. We tested on individual sources the difference between flagging the baselines compared to tapering and found the differences to be minimal; there was no difference in the final RM map area, and the differences in RM values were a fraction of the error bars. Additionally, we restored all the maps to the beam size of our lowest frequency $(8.1 \mathrm{GHz})$. All these steps were carried out in Difmap after the initial data reduction and self-calibration, which were done using the full $(u, v)$ data.

\subsection{Absolute EVPA Calibration}

The absolute EVPA offset is an instrumental quantity that must be determined and applied to every VLBA polarization observation. To calibrate the EVPAs of our data, we used Very Large Array (VLA), University of Michigan Radio Astronomy Observatory (UMRAO), and $15 \mathrm{GHz}$ VLBA data, and instrumental leakage term (D-term) phases. The $15 \mathrm{GHz}$ observations were previously calibrated as part of the MOJAVE project using the D-term calibration method (Gómez et al. 2002; Lister \& Homan 2005). Therefore, we only had to calibrate the 8 and $12 \mathrm{GHz}$ bands. For five epochs we were able to use the VLA/VLBA polarization calibration database ${ }^{11}$ to find polarization observations within a week of our epoch and including one or two of our sources. For those epochs, we also calculated the distribution of differences between the calibrated $15 \mathrm{GHz}$ EVPAs and other bands, and UMRAO 8 and $15 \mathrm{GHz}$ EVPAs versus our 8 and $15 \mathrm{GHz}$ EVPAs. Usually these difference histograms showed a peak at an angle similar to that determined from the VLA observations. The typical errors in the

\footnotetext{
11 http://www.aoc.nrao.edu/ smyers/calibration/
}

Table 2

EVPA Calibration Corrections for All Epochs in Degrees

\begin{tabular}{llrrrrr}
\hline \hline Obs. Code & \multicolumn{1}{c}{ Epoch } & Ref. Ant. & $8.1 \mathrm{GHz}$ & $8.4 \mathrm{GHz}$ & $12.1 \mathrm{GHz}$ & $15.4 \mathrm{GHz}^{\mathrm{b}}$ \\
\hline BL137A & 2006 Feb 2 & PT & -15.9 & -16.7 & -16.1 & -18.8 \\
BL137B $^{\mathrm{a}}$ & 2006 Mar 6 & PT & -17.7 & -20.0 & -18.0 & -14.5 \\
BL137C & 2006 Apr 5 & KP & 19.2 & 13.1 & 22.5 & 30.9 \\
BL137D & 2006 Apr 28 & FD & 12.4 & 6.7 & -42.9 & -53.7 \\
BL137E & 2006 May 24 & FD & -12.8 & -17.4 & -16.7 & -47.3 \\
BL137F $^{\mathrm{a}}$ & 2006 Jun 15 & FD & -42.2 & -47.3 & -47.1 & -49.2 \\
BL137G $^{\mathrm{a}}$ & 2006 Jul 7 & FD & -46.3 & -47.9 & -47.9 & -49.1 \\
BL137H $^{2}$ 2006 Aug 9 & FD & -45.0 & -47.8 & -47.0 & -48.4 \\
BL137I $^{\mathrm{a}}$ & 2006 Sep 6 & PT & -10.0 & -10.0 & -14.3 & -14.9 \\
BL137J $^{2}$ 2006 Oct 6 & FD & -45.3 & -46.8 & -45.6 & -48.3 \\
BL137K & 2006 Nov 10 & FD & -44.0 & -45.0 & -45.0 & -46.6 \\
BL137L & 2006 Dec 1 & FD & -44.2 & -47.1 & -47.4 & -50.5 \\
\hline
\end{tabular}

Notes.

${ }^{a}$ Epoch used to anchor the D-term calibration.

${ }^{\mathrm{b}}$ Calibrated as part of the MOJAVE project.

VLA EVPAs range from $1^{\circ}$ to $3^{\circ}$ and in the UMRAO data from $1^{\circ}$ to $10^{\circ}$, but these cancel when multiple sources are used.

By using these five epochs, we were able to find D-term phases on various antennas that were stable enough over the 12 month period to enable the use of D-term phases in the calibration of the EVPAs of the remaining epochs. The EVPA corrections for all the epochs are shown in Table 2 where in column (1) we give the observing code of the epoch and list the epochs that were used to anchor the D-terms. The epoch of observations is listed in column (2), and the reference antenna used in the calibration in column (3). The EVPA corrections at $15.4,12.1,8.4$, and $8.1 \mathrm{GHz}$ are given in columns (4)-(7). Since we are using five different anchoring epochs with different VLA calibration sources in addition to the UMRAO data, the main source of error in our calibration method should be the scatter in the measured D-term phases. By calculating the standard deviation of the mean for the scatter in each right-hand or lefthand phase and taking the maximum value over the frequency band as a conservative error estimate, we determine the absolute EVPA calibration errors to be $3^{\circ}, 2^{\circ}$, and $4^{\circ}$ at 15,12 , and $8 \mathrm{GHz}$ bands, respectively. The total error in the EVPAs is a quadrature sum of the calibration error and statistical error in the EVPA, with the latter being derived from the rms values in $Q$ and $U$ maps. 
The error in the final rotation measure images is highly dependent on the error of the EVPA. We have performed detailed simulations to verify that our error estimate, derived with error propagation from the rms in $Q$ and $U$ images, is correct. These simulations are described in detail in Appendix B, where we also give the equations used in the error calculation.

\subsection{Image Alignment}

During the initial data reduction process the absolute coordinate position of the source is lost and the center of the image is shifted to the phase center of the map. This may not be the same position on the sky for different frequency bands, and therefore an extra step is needed to align the images. This can be done using bright components in the optically thin part of the jet, whose position should not depend on the observing frequency (e.g., Lobanov 1998; Marr et al. 2001; Kovalev et al. 2008; Sokolovsky et al. 2011). This approach works well for knotty jets but is unreliable or impossible to use for faint or smooth jets. A solution is to use a two-dimensional (2D) cross-correlation algorithm to look for the best alignment based on correlation of the optically thin parts of the jets at different bands (e.g., Walker et al. 2000; Croke \& Gabuzda 2008).

We used both methods whenever possible, and concluded that the results matched very well when using bright optically thin components. Similar to, e.g., Marr et al. (2001) and Kovalev et al. (2008), all the shifts were verified by examining the spectral index maps before and after the alignment. In shifted maps the spectral index gradient along the jet was typically smoother, and any optically thin regions apparently upstream of the core disappeared. The absolute shifts between $15 \mathrm{GHz}$ and other bands varied between 0 mas and 2.02 mas with a median value of 0.11 mas. This is comparable to the pixel size of 0.1 mas used in the RM images. The extreme value of 2.02 mas is for the source $2134+004$ between $15 \mathrm{GHz}$ and $12 \mathrm{GHz}$, where a different component is the brightest feature in the two maps. This illustrates the importance of correct alignment for the data analysis. The small median shift, however, shows that in the majority of the sources the change is not extreme as is to be expected for bright, core-dominated objects. These shifts are determined in part by the frequency-dependent core shifts, which will be studied in our sample in Pushkarev et al. (2012), although other effects can also contribute in some cases, such as in 2134+004 described above.

For 35 sources we were not able to find a reliable alignment due to the compactness of the source or the faintness of a featureless jet. In these cases we aligned the images based on the fitted core component position at each band. The median shift values for these sources were less than 0.03 mas. We used spectral index maps to verify that our alignments were reasonable. The spectral index maps of all the sources will be presented and discussed in a separate paper (T. Hovatta et al., in preparation).

Additionally, we did several tests, described in Appendix A, to study the effect of false alignment on spectral index and rotation measure maps. Based on the tests we conclude that even if our image alignment is off by 0.15 mas between $15 \mathrm{GHz}$ and any other frequency band, it should not affect the results from our rotation measure maps, especially as we are not using the edge or low signal-to-noise regions to make conclusions about the RM structure. We verify that the spectral index map is a good indicator of the image alignment because the effect of small fake shifts can readily be seen in the structure of the spectral index map.

\subsection{Rotation Measure Maps}

For the calculation of the RM maps we wrote a Perl Data Language (PDL) script that does the calculation semi-automatically for our large sample of sources. We verified the performance of the script by using the RM task manually in AIPS for several sources. In our calculations we blanked all the pixels that had polarized flux density less than three times the polarization error, defined in Appendix B, at any of the frequency bands. Our script chooses the best $\lambda^{2}$-fit based on a $\chi^{2}$ criterion and blanks all the pixels where it is not met. We calculate the $\chi^{2}$ of the fit using the standard formulae:

$$
\chi^{2}=\sum_{i=1}^{N} \frac{\left(O_{i}-E_{i}\right)^{2}}{\sigma_{i}^{2}}
$$

where $N$ is the number of data points, $O_{i}$ are the observed data, $E_{i}$ are the expected data based on the model, and $\sigma_{i}$ is the measurement error of the individual data point (e.g., Press et al. 1992). Due to the dependence on the errors of the data points, blanking of low signal-to-noise regions is essential to prevent small $\chi^{2}$ values simply due to large error bars. In general care must be taken in determining the EVPA errors because errors that are too small will prevent good fits while errors that are too large will result in $\chi^{2}$ values that are too small. Our EVPA errors are estimated by adding an rms error in quadrature using error propagation from $Q$ and $U$ images (see Appendix B for details) and an absolute calibration error defined in Section 2.2. As we are fitting a two-parameter model to four data points we have 2 degrees of freedom and from a $\chi^{2}$ distribution the corresponding $95 \%$ confidence limit is $\chi^{2}<5.99$.

The EVPA is ambiguous for changes of $180^{\circ}$ and in the calculation of the RM we need to solve for these $n \pi$-wraps. We first assumed that there are no $n \pi$-wraps between our frequency bands and calculated the RM fit. If the $\chi^{2}$ of the fit met our criterion, we accepted the RM value without any wraps. If the $\chi^{2}$ criterion is not met, we solved for all possible $n \pi$-wraps up to $3.3 \times 10^{4} \mathrm{rad} \mathrm{m}^{-2}$ and chose the fit with the smallest wrap meeting our $\chi^{2}$ criterion. The upper limit was primarily introduced to keep the computing time reasonable but also because based on earlier studies (e.g., Zavala \& Taylor 2003, 2004), we did not expect to resolve RMs larger than this with our frequency setup. If none of the wraps resulted in acceptable fits, we blanked the pixel. By blanking the poor $\lambda^{2}$-fit regions, we prevent interpretations based on noisy data and identify regions with non- $\lambda^{2}$-law behavior.

The error of the RM is calculated from the variancecovariance matrix of the least-squares fit in each pixel. Our typical errors range between 70 and $150 \mathrm{rad} \mathrm{m}^{-2}$ depending on the signal-to-noise ratio of the total intensity in the jet, thus in the fainter jet edges the RM errors are larger. We verified that our error estimates are correct by performing detailed simulations described in Appendix C.

In order to study the distribution of the intrinsic, redshiftcorrected, RM values, the Galactic Faraday rotation contribution must be taken into account. We used the averaged Galactic RM image of Taylor et al. (2009) and subtracted the value at the source location from each map. We list the values used for each source in Table 1. In the majority of sources the Galactic Faraday rotation is very small (the median absolute value for the sample is $12.3 \mathrm{rad} \mathrm{m}^{-2}$ ) but there are 14 sources for which the absolute value is more than $70 \mathrm{rad} \mathrm{m}^{-2}$, thereby exceeding our minimum error in the RM values. The largest absolute Galactic Faraday 

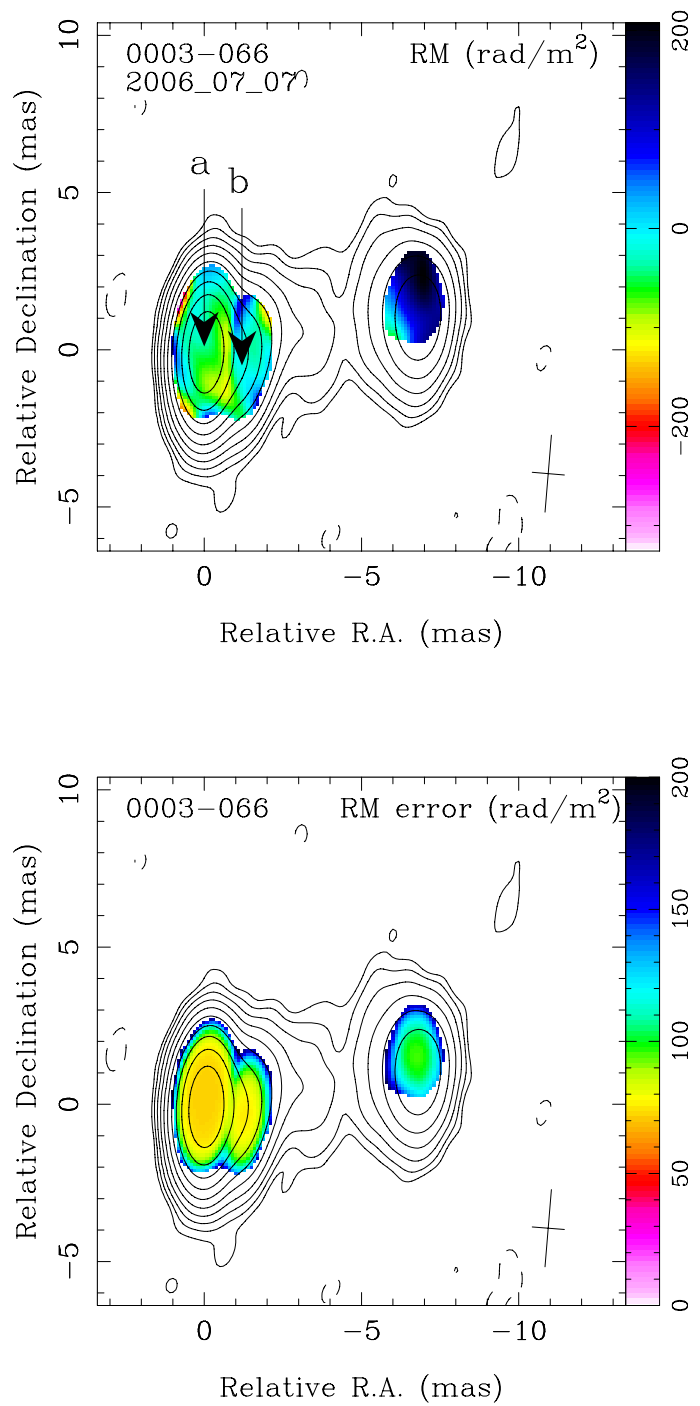
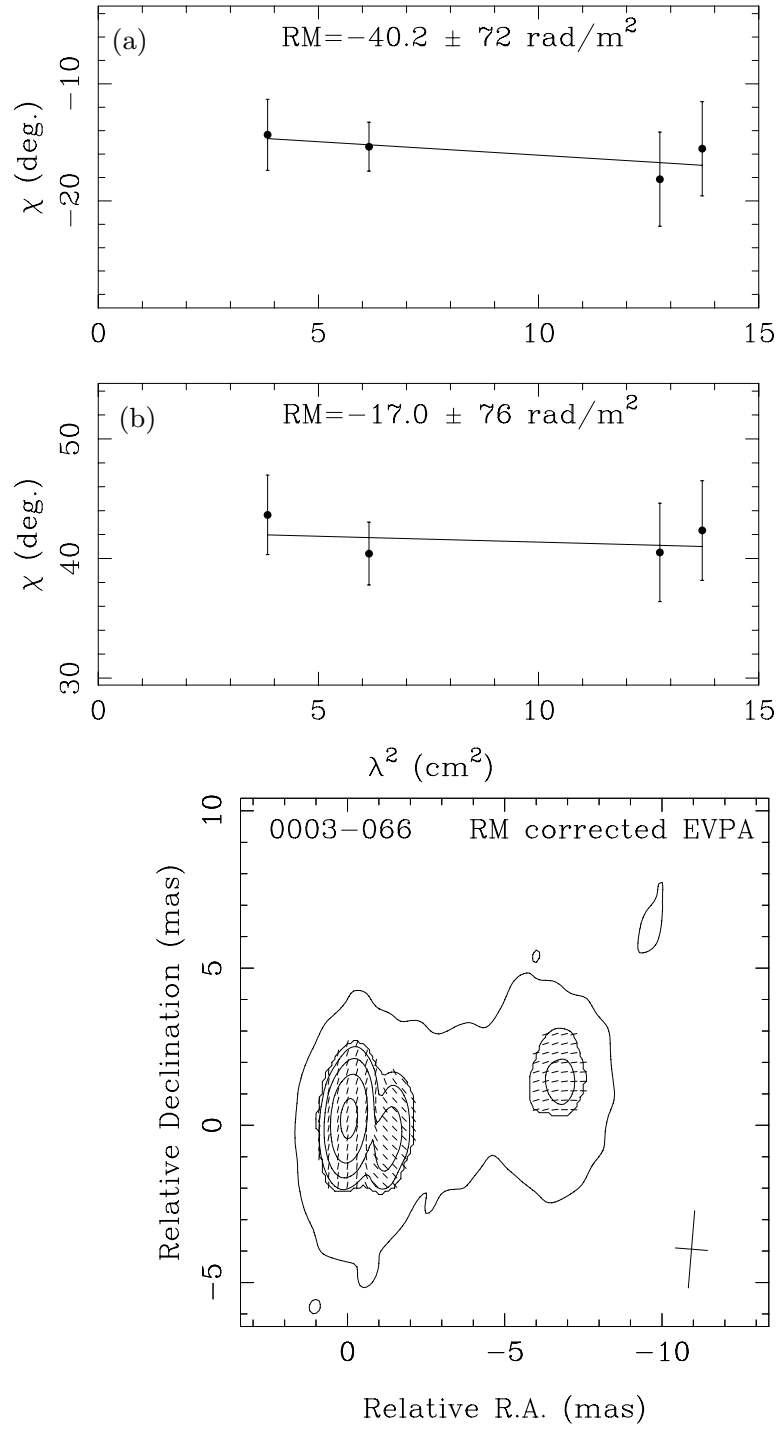

Figure 1. Rotation measure maps of all the sources. RM map (top left) with $\lambda^{2}$-fits at one or two locations in the jet (top right). Error map (bottom left) and map of rotated EVPA values (bottom right).

(The complete figure set (159 images) and color version of this figure are available in the online journal.)

rotation values are observed for $2021+317\left(-173 \mathrm{rad} \mathrm{m}^{-2}\right)$ and $2200+420\left(-156 \mathrm{rad} \mathrm{m}^{-2}\right)$. For the majority of sources in our sample the values from Taylor et al. (2009) agree very well with previously published values (Rudnick \& Jones 1983; Rusk 1988; Wrobel 1993; Pushkarev 2001). However, we note that since we are using an averaged image, some Galactic RM values may be underestimated because small regions of high Galactic RM get smoothed out (e.g., 0235+164, 1749+096, 1803+784, $2200+420)$.

\section{RESULTS}

RM maps are shown in Figure 1 for the 159 cases where we detect enough polarization to get an RM value for at least a few pixels. We show the RM values in color scale overlaid on the $15 \mathrm{GHz}$ total intensity contours and examples of the $\lambda^{2}$-fits in two locations of the jet, chosen to be at the polarization peaks of the map. These locations are typically at least one beam width apart. In sources where clear polarization peaks were not seen, we chose the location to be in the middle of the $\mathrm{RM}$ region. Additionally, we show the error of the RM in color and the intrinsic, RM corrected, $15 \mathrm{GHz}$ polarization vectors overlaid on the $15 \mathrm{GHz}$ polarization contours. All the RM maps in Figure 1 and later in the paper are corrected for Galactic Faraday rotation. In some cases, there appear to be pixels with very high $\mathrm{RM}$ values of over $\pm 2 \times 10^{4} \mathrm{rad} \mathrm{m}^{-2}$. In most of the sources these coincide with edge pixels and/or regions of complex polarization structure. Our simulations of the RM error (see Appendix C) show that it is possible to have spurious high-RM pixels in the maps purely due to random noise in the polarization images. These were always more than $\pm 2 \times 10^{4} \mathrm{rad} \mathrm{m}^{-2}$ and in our real maps the high-RM regions resembled the simulated maps very well. Therefore, we have blanked these extreme values in Figure 1.

\subsection{Extreme RM Values}

In some sources we see very high RM regions around the core where we may expect more Faraday rotating material and stronger magnetic fields. Udomprasert et al. (1997) report an intrinsic $\mathrm{RM}\left(\mathrm{RM}_{\text {int }}\right)$ of $4 \times 10^{4} \mathrm{rad} \mathrm{m}^{-2}$ in the high-redshift quasar OQ $172(z=3.52)$. In the observed frame, defined as $\mathrm{RM}_{\mathrm{obs}}=(1+z)^{-2} \mathrm{RM}_{\text {int }}$, this corresponds to an $\mathrm{RM}_{\mathrm{obs}}$ of $\sim 2000 \mathrm{rad} \mathrm{m}^{-2}$ in the core. If the intrinsic value is correct, 

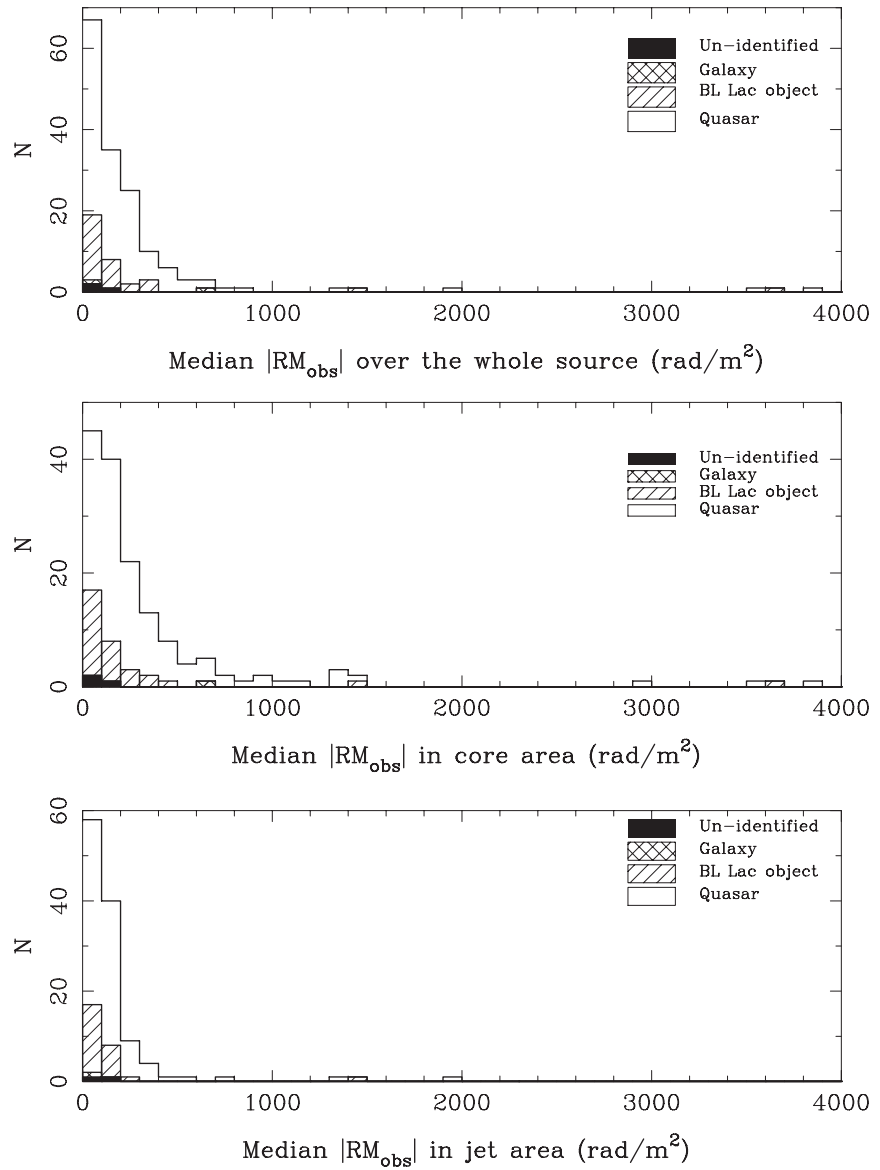

Figure 2. Distribution of median $\left|\mathrm{RM}_{\mathrm{obs}}\right|$ over the whole source (top), over the core region (middle), and over the jet region (bottom). Quasars are shown in white, BL Lac objects in hatched, galaxies in cross-hatched, and unidentified sources in black. These plot excludes quasars $0003+380$ and 2008-159 which have $\mathrm{RM}$ values over $6000 \mathrm{rad} \mathrm{m}^{-2}$.

we might expect to observe extremely high RMs in some nearby objects. Attridge et al. (2005) report a difference of $\sim 3.2 \times 10^{4} \mathrm{rad} \mathrm{m}^{-2}$ between two components in the core of 3C 273 in observations at 43 and $86 \mathrm{GHz}$. It is, however, very difficult at our observing frequencies with much less resolution to distinguish true extremely high $\mathrm{RM}_{\mathrm{obs}}$ values from the spurious ones due to noise and blending of components. For example, in 3C 273 we observe extreme $\mathrm{RM}_{\mathrm{obs}}$ values of $\sim 2.3 \times 10^{4} \mathrm{rad} \mathrm{m}^{-2}$ around the core in the March epoch. In our June epoch, we do not detect these high values but instead see values of $\sim-2.9 \times 10^{4} \mathrm{rad} \mathrm{m}^{-2}$ around the same region. Similar behavior is seen in the cores of 3C 279 and 3C 454.3. In $2200+420$ we do not find good $\lambda^{2}$-fits in the core in our April epoch but in November we detect extreme $\mathrm{RM}_{\mathrm{obs}}$ values of $\sim+2.9 \times 10^{4} \mathrm{rad} \mathrm{m}^{-2}$, never seen before in this source (Mutel et al. 2005; Zavala \& Taylor 2003) including observations of O'Sullivan \& Gabuzda (2009) in 2006 July. Other sources where we detect extreme $\mathrm{RM}_{\text {obs }}$ values in larger areas near the core include 0149+218, 0420-014, 0605-085, 1038+064, and $2145+045$. Out of these, 0420-014 was observed by Zavala \& Taylor (2003) who do not see these extreme values although they also do not find a good $\lambda^{2}$-fit at the core component position. 0605-085 was observed by Zavala \& Taylor (2004) who do not detect any extreme RM values and find the RM in the core to follow the $\lambda^{2}$-law. In $2145+067$ the polarization structure at $15 \mathrm{GHz}$ is extremely complex with four separate components seen within the innermost jet, while at $8 \mathrm{GHz}$ only one component is seen. Therefore, we do not believe that these extreme observed core RMs are real in our maps, but instead are due to multiple polarized components blending in the finite beam or due to different opacity properties at the frequency bands. This is further supported by continuing MOJAVE observations of $2200+420$ which show a new component emerging from the core in 2007 February. ${ }^{12}$ Algaba et al. (2011) observe high RMs in the cores of several sources in their study of eight sources between 12 and $43 \mathrm{GHz}$. They observe an $\mathrm{RM}_{\mathrm{obs}}$ of $2.2 \times 10^{4} \mathrm{rad} \mathrm{m}^{-2}$ in the core of $1633+382$, for which we observe a small $\mathrm{RM}_{\mathrm{obs}}$ of $-244 \mathrm{rad} \mathrm{m}^{-2}$. Their result is based on a large difference in the EVPAs of the 22 and $24 \mathrm{GHz}$ observations which require a large RM. It is possible that they are able to resolve structures not seen in our maps due to their higher resolution. In some sources they also find that they need to divide the frequency range into high- and low-frequency parts to obtain acceptable $\lambda^{2}$-fits, which is a further indication of different frequencies probing different regions in the core and multiple components blending within the beam in the lower frequency maps.

The blending of components in the core region can also affect our $\lambda^{2}$-fits so that the $\chi^{2}$ criterion is not met and no RM values are shown in the maps. Another cause for this could be internal Faraday rotation, which could play a significant role in the AGN core regions. We also see non- $\lambda^{2}$ patches in the jets of some sources, sometimes due to the faintness of the jet emission, but at other times also due to depolarization of the lower frequencies, a sign of internal Faraday rotation. The effects of internal Faraday rotation and other depolarization mechanisms are discussed in more detail in Section 4.2.

\subsection{Median RM Distribution}

We were able to determine the median $\mathrm{RM}_{\text {obs }}$ for 159 maps, which are shown in the top panel of Figure 2 and given in Table 1, where column (1) gives the B1950-name of the source and column (2) an alternative alias name. The redshift and the optical classification of the source are listed in columns (3) and (4). Apparent speed used for calculation of the de-projected distance in Section 4.1 is given in column (5) and the observing epoch is listed in column (6). The value used for Galactic Faraday rotation correction, taken from Taylor et al. (2009) is listed in column (7). The median $\mathrm{RM}_{\mathrm{obs}}$ value, taken as the median of all the pixels in the source where RM is detected and not blanked, is listed in column (8). Columns (9) and (10) give the median RM over the core and the jet regions, respectively. We calculate the median instead of the average to lessen the effect of individual, possibly spurious, high-RM values. The vast majority of sources have a median $\mathrm{RM}_{\mathrm{obs}}$ of less than $1000 \mathrm{rad} \mathrm{m}^{-2}$, but the distribution has a tail to $\mathrm{RM}_{\mathrm{obs}}$ values of $6500 \mathrm{rad} \mathrm{m}^{-2}$. The highest value shown in the plot, $6457 \mathrm{rad} \mathrm{m}^{-2}$, is for the source $2008-159$, which only shows $\mathrm{RM}$ values in a small region of less than half the beam size. At the redshift 1.18 of the source, this would result in an extremely high intrinsic $\mathrm{RM}_{\mathrm{int}}$ of over $3 \times 10^{4} \mathrm{rad} \mathrm{m}^{-2}$ in the source frame. As the region over which we detect the high $\mathrm{RM}_{\mathrm{obs}}$ value is so small and does not coincide with any total intensity component locations, it is difficult to say if this is a true RM of the source or due to blending of multiple components within the core region.

\footnotetext{
12 http://www.physics.purdue.edu/astro/MOJAVE/sourcepages/ 2200+420.shtml
} 
Table 3

Model-fit Components at $15.3 \mathrm{GHz}$ and Their $\mathrm{RM}_{\mathrm{obs}}$ Values

\begin{tabular}{lcccccc}
\hline \hline Source & ID & Epoch & $\begin{array}{c}r \\
(\mathrm{mas})\end{array}$ & $\begin{array}{c}\text { P.A. } \\
(\mathrm{deg})\end{array}$ & $\begin{array}{c}\mathrm{RM} \\
\left(\mathrm{rad} \mathrm{m}^{-2}\right)\end{array}$ & Isolated \\
$(1)$ & $(2)$ & $(3)$ & $(4)$ & $(5)$ & $(6)$ & $(7)$ \\
\hline $0003-066$ & 0 & 2006 Jul 7 & 0.71 & -168.6 & $-78 \pm 73$ & \\
$0003-066$ & 1 & 2006 Jul 7 & 0.66 & -71.6 & $-88 \pm 75$ & \\
$0003-066$ & 4 & 2006 Jul 7 & 6.86 & -81.1 & $113 \pm 98$ & $\mathrm{Y}$ \\
$0003-066$ & 5 & 2006 Jul 7 & 0.09 & 2.9 & $-42 \pm 72$ & \\
$0003-066$ & 6 & 2006 Jul 7 & 1.28 & -102.7 & $-14 \pm 77$ & \\
$0007+106$ & 1 & 2006 Jun 15 & 0.39 & -66.6 & $627 \pm 130$ & \\
$0016+731$ & 0 & 2006 Aug 9 & 0.01 & -52.2 & $278 \pm 74$ & \\
$0016+731$ & 2 & 2006 Aug 9 & 0.20 & 129.2 & $266 \pm 74$ & \\
\hline
\end{tabular}

Notes. Columns are as follows: (1) IAU Name (B1950.0); (2) ID of the component ( $0=$ core); (3) observing epoch; (4) component distance from the phase center of the I map; (5) position angle of the component from the phase center; (6) component RM; (7) flag for isolated jet components.

(This table is available in its entirety in machine-readable and Virtual Observatory (VO) forms in the online journal. A portion is shown here for guidance regarding its form and content.)

\subsection{Core versus Jet Distributions}

To study the difference between core and jet RM values, we (1) divided the source into core and jet regions by defining the core region to be everything within a beam width from the center of the $15 \mathrm{GHz}$ core component position and jet region to be everything else and (2) took the $15 \mathrm{GHz}$ model-fit components (see Lister et al. 2009b for details on the model-fitting) and divided the source into core and jet components. In the first approach the division is determined by a line perpendicular to the jet direction at one beam width away from the core. Median values over the pixels within the regions were calculated and are given in Table 1 (columns (9) and (10)) and shown in the bottom two panels of Figure 2.

In the second approach, we calculated the average RM over the 9 contingent pixels around the component position to avoid basing conclusions on single pixel values. This corresponds to $10 \%-30 \%$ of the restoring beam width depending on the declination of the source. The component locations and their $\mathrm{RM}_{\mathrm{obs}}$ values are given in Table 3. The ID number of the component is listed in column (2) where 0 indicates a core component. Columns (4) and (5) give the component distance and position angle from the phase center of the map. The $\mathrm{RM}_{\mathrm{obs}}$ and its error are given in column (6). Because the pixels are not independent (i.e., they cover a region smaller than the FWHM of the restoring beam), it is not straightforward to estimate the error on the average carried out over 9 pixels. We define the error as the average of RM errors in the 9 individual pixels; this approach is conservative, and may overestimate the true error somewhat. Column (7) indicates whether a jet component is isolated (see below). Most of the sources in the MOJAVE sample are core dominated, with a bright compact core that is optically thick at centimeter wavelengths and a fainter jet. In most of our sources, we identify the core as a bright, stationary feature in the jet, typically at one extreme end of the jet. In a few sources (especially with two-sided jets) the identification is not as simple and these are discussed separately in Lister et al. (2009b).

The distribution of the $\mathrm{RM}_{\mathrm{obs}}$ values in the components is shown in Figure 3. We were able to determine the core component RM in 104 maps (101 sources) and the jet RM in 324 components (121 sources). From the distributions it
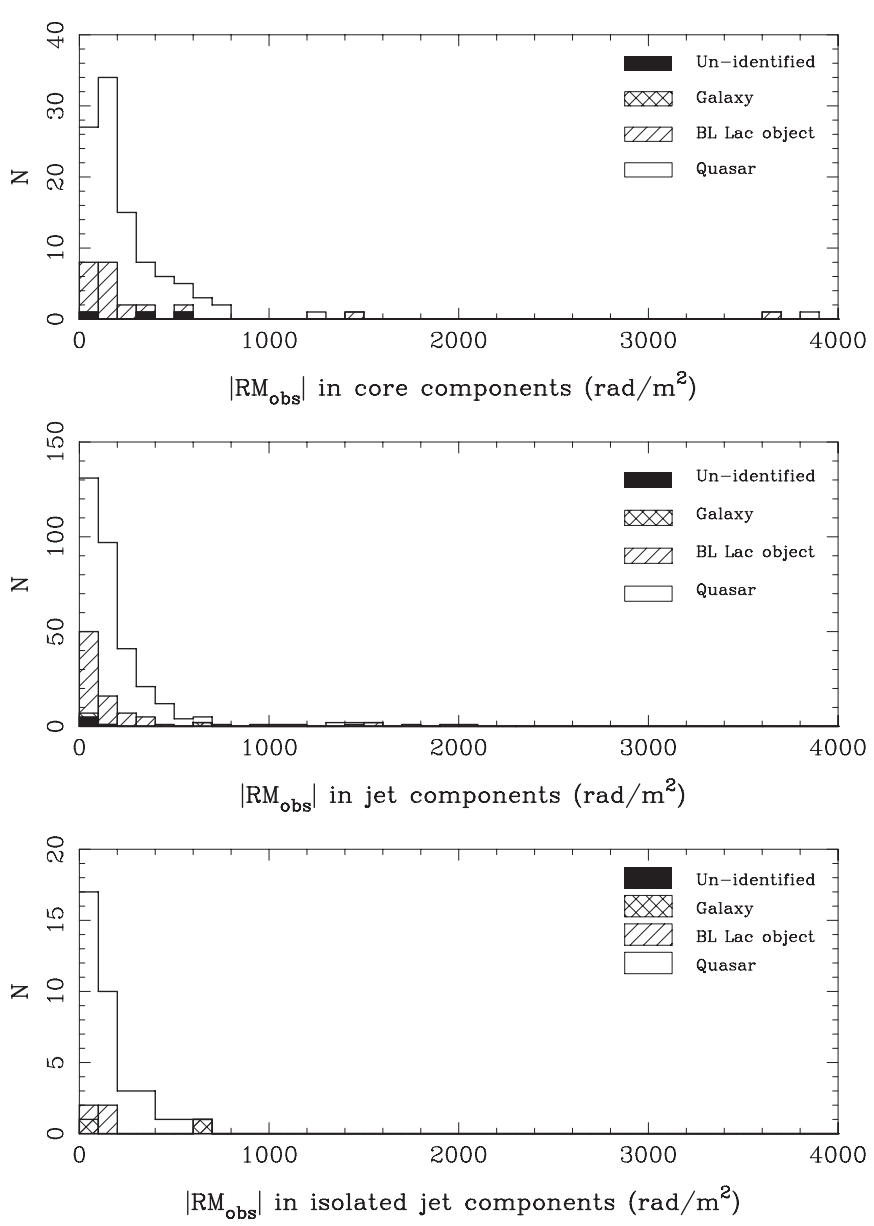

Figure 3. Distributions of $\left|\mathrm{RM}_{\mathrm{obs}}\right|$ in the model-fit core (top) and jet (middle) components. Jet components which have less than $30 \%$ total intensity contribution from other components in the source are plotted in the bottom panel (see the text for details). Quasars are shown in white, BL Lac objects in hatched, galaxies in cross-hatched, and unidentified sources in black.

is clear that the core component values have a tail to higher $\mathrm{RM}_{\mathrm{obs}}$ values, but there are also some jet components with high $\mathrm{RM}_{\mathrm{obs}}$ values. In most of those cases the jet component is within 1 mas of the core component and often still in the optically thick or self-absorbed region of the jet. To distinguish the jet components which are away from the bright core region, for each component we calculated the combined contribution of all the other jet components in the map at the component's peak intensity position. If this sum was less than $30 \%$ of the component's total intensity, we considered the component to be isolated. In this way we determined that the polarization and RM of the component were not affected by nearby bright components. Out of all the jet components, 36 in 24 sources are listed as isolated. The distribution of $\mathrm{RM}_{\mathrm{obs}}$ values of these isolated components is plotted in the bottom panel of Figure 3. As can be seen, none of the $\mathrm{RM}_{\mathrm{obs}}$ values greater than $700 \mathrm{rad} \mathrm{m}^{-2}$ are isolated. The median component $\mathrm{RM}_{\mathrm{obs}}$ in the whole sample is $171 \mathrm{rad} \mathrm{m}^{-2}$ for the cores, $125 \mathrm{rad} \mathrm{m}^{-2}$ in all the jet components and $104 \mathrm{rad} \mathrm{m}^{-2}$ in the isolated jet components. According to an Anderson-Darling (A-D) two-sample test (e.g., Press et al. 1992), which is more sensitive to distribution tails than the Kolmogorov-Smirnov test, the core and jet components have less than a $1 \%$ probability of coming from the same parent population. Comparison of core and isolated jet components gives a probability of less than $2 \%$ due to the smaller number of 
jet components. In all our tests we consider the result significant if the probability is less than $5 \%$.

A similar trend is seen when comparing the core and jet regions in Figure 2. The median RM for the core regions is $187 \mathrm{rad} \mathrm{m}^{-2}$ and for the jets is $102 \mathrm{rad} \mathrm{m}^{-2}$, a result very similar to that for the isolated jet components. According to the A-D test, the probability for these distributions to come from the same parent population is less than $0.001 \%$.

\subsection{Optical Subclasses}

Our sample can also be divided into subclasses based on the optical classification of the source. These are also shown in Figures 2 and 3. The number of galaxies and optically unidentified sources in our sample is so small that they cannot be included in any statistical comparisons. The quasars and BL Lac objects, however, can be compared, and it is clear that the high-RM tail in the distributions consists mainly of quasars. The median absolute $\mathrm{RM}_{\mathrm{obs}}$ value in quasars is $144 \mathrm{rad} \mathrm{m}^{-2}$ and in the BL Lac objects $79 \mathrm{rad} \mathrm{m}^{-2}$.

If we look at the core and jet components individually, there is a less than $0.1 \%$ probability that the jet components of quasars and BL Lac objects are drawn from the same population. The median jet $\mathrm{RM}_{\mathrm{obs}}$ for quasars is $141 \mathrm{rad} \mathrm{m}^{-2}$ while for the $\mathrm{BL}$ Lac objects it is $71 \mathrm{rad} \mathrm{m}^{-2}$. However, this difference is affected by the components within one beam width of the core in quasars because the median values in the jet regions of quasars is $116 \mathrm{rad} \mathrm{m}^{-2}$ and in BL Lac objects it is $76 \mathrm{rad} \mathrm{m}^{-2}$. According to the A-D test, we cannot reject the null hypothesis that the distributions come from the same parent population.

In the cores differing results are also obtained when core components and regions are compared. In quasars the median core component value is $183 \mathrm{rad} \mathrm{m}^{-2}$ compared to $134 \mathrm{rad} \mathrm{m}^{-2}$ in BL Lac objects, and we cannot reject the null hypothesis that they come from the same parent population. In the core regions, however, the median for quasars is $200 \mathrm{rad} \mathrm{m}^{-2}$ and for BL Lac objects it is $105 \mathrm{rad} \mathrm{m}^{-2}$, and there is a less than $1 \%$ probability that these come from the same distribution.

These results can be compared to Zavala \& Taylor (2004), who saw a difference in the core RMs for quasars and BL Lac objects but not in the jet values. Our results on the jet RMs agree if we look at the jet regions which are not contaminated by components near the core. We cannot verify whether there is a difference between the cores in quasars and BL Lac objects because of the differing results depending on if we look at the core components or core regions. However, the higher median values observed in quasars than in BL Lac objects are in accordance with the standard models in which BL Lac objects have less material around them, resulting in dimmer and narrower emission lines than in quasars.

When examining the intrinsic, redshift corrected, $\mathrm{RM}_{\mathrm{int}}$ values in the components the difference between BL Lac objects and quasars is more significant. This is mainly due to the BL Lac objects in our sample having smaller redshifts than the quasars (median redshift 0.31 versus 1.12 ), which is enhanced in the correction defined as $\mathrm{RM}_{\text {int }}=\mathrm{RM}_{\mathrm{obs}}(1+z)^{2}$, where $z$ is the redshift.

The absolute $\mathrm{RM}_{\text {int }}$ values in the cores of quasars range from 4.8 to $6436 \mathrm{rad} \mathrm{m}^{-2}$ with a median of $798 \mathrm{rad} \mathrm{m}^{-2}$. In $\mathrm{BL}$ Lac objects the range of the core $\mathrm{RM}_{\text {int }}$ values is from 13 to $3873 \mathrm{rad} \mathrm{m}^{-2}$, similar to the quasars, but the median is significantly smaller $\left(274 \mathrm{rad} \mathrm{m}^{-2}\right)$ and according to an A-D test the probability for the two distributions to come from the same parent population is less than $3 \%$. The median $\mathrm{RM}_{\text {int }}$ value for the jet components of BL Lac objects (ranging from 0.8 to $1937 \mathrm{rad} \mathrm{m}^{-2}$ with a median of $148 \mathrm{rad} \mathrm{m}^{-2}$ ) is significantly smaller than that of the cores even though the range is similar. In quasars they range from 1 to $8975 \mathrm{rad} \mathrm{m}^{-2}$ with a median of $563 \mathrm{rad} \mathrm{m}^{-2}$, but the probability of rejecting the null hypothesis in the case of the intrinsic jet and core components of quasars is only $6.8 \%$ and therefore not significant. Similar to the case of core components, the difference between quasars and BL Lac objects is significant also in the intrinsic jet components.

\section{DISCUSSION}

One of the main scientific motivations for the multifrequency survey of the MOJAVE sources was to determine the effects of Faraday rotation on the observed polarization structure of the sources at $15 \mathrm{GHz}$. Based on the first epoch MOJAVE data, Lister \& Homan (2005) showed that in BL Lac objects the distribution of EVPAs with respect to the local jet direction appears bimodal. The effect of Faraday rotation was not taken into account and therefore these results could be affected by sources with high RM values. The RM distribution of Figure 2 shows that in over $80 \%$ of our sources the $\mathrm{RM}_{\mathrm{obs}}$ values are less than $400 \mathrm{rad} \mathrm{m}^{-2}$, which will rotate the $15 \mathrm{GHz}$ electric vectors by about $10^{\circ}$. This means that the results of a large sample of Lister \& Homan (2005) should approximately reflect the true distribution at $15 \mathrm{GHz}$. However, when studying some individual sources, the Faraday rotation must be taken into account as a rotation measure of $2000 \mathrm{rad} \mathrm{m}^{-2}$ (seen in the median RM distributions for a few individual sources) can rotate the $15 \mathrm{GHz}$ EVPAs by $40^{\circ}$. For example, in $0429+415$ we detect $\mathrm{RM}_{\mathrm{obs}}$ of $\sim 1900 \mathrm{rad} \mathrm{m}^{-2}$ in several jet components 40 mas from the core, similarly to Mantovani et al. (2010). In $1101+384$ and $1725+044$ we detect core component $\mathrm{RM}_{\mathrm{obs}}$ as high as $3800 \mathrm{rad} \mathrm{m}^{-2}$, although it must be noted that in these two sources we detect RM only in a very small region around the core and therefore we cannot be sure whether it is true RM or due to blending of multiple polarized components within the finite beam.

\subsection{Distance Dependence}

In Figure 4 (top panel) we show the $\mathrm{RM}_{\mathrm{obs}}$ versus projected distance from the core for all the model-fit jet components. The dependence for the total sample is not very clear although according to a non-parametric Kendall's $\tau$ correlation test (e.g., Press et al. 1992) there is a significant negative correlation ( $\tau=-0.13, p=0.00058$ ). When quasars and BL Lac objects are studied separately it can be seen that the correlation in quasars is stronger $\left(\tau=-0.17, p=6.3 \times 10^{-5}\right)$ while for the BL Lac objects alone $\tau=-0.21, p=0.02$. The picture is, however, more complicated as the true distance from the core depends on the viewing angle of the source. We have therefore de-projected the distances using viewing angles $\theta=\tan ^{-1}\left[\left(2 \beta_{\text {app }}\right) /\left(\beta_{\text {app }}^{2}+D_{\text {var }}^{2}-1\right)\right]$ determined with Doppler factors $D_{\text {var }}$ from Hovatta et al. (2009) and apparent speeds $\beta_{\text {app }}$ from Lister et al. (2009b). Some of the speeds have been updated since Lister et al. (2009b), and all the speeds used are tabulated in Table 1. Both values were available for 138 components in quasars, 47 components in BL Lac objects, and 4 jet components in galaxies. The $\mathrm{RM}_{\mathrm{obs}}$ against the deprojected distance is shown in Figure 4 (bottom panel). The negative correlation in quasars remains significant despite the smaller number of sources $\left(\tau=-0.23, p=4.8 \times 10^{-5}\right)$ while in BL Lac objects the correlation vanishes $(\tau=-0.01, p=0.92)$. 

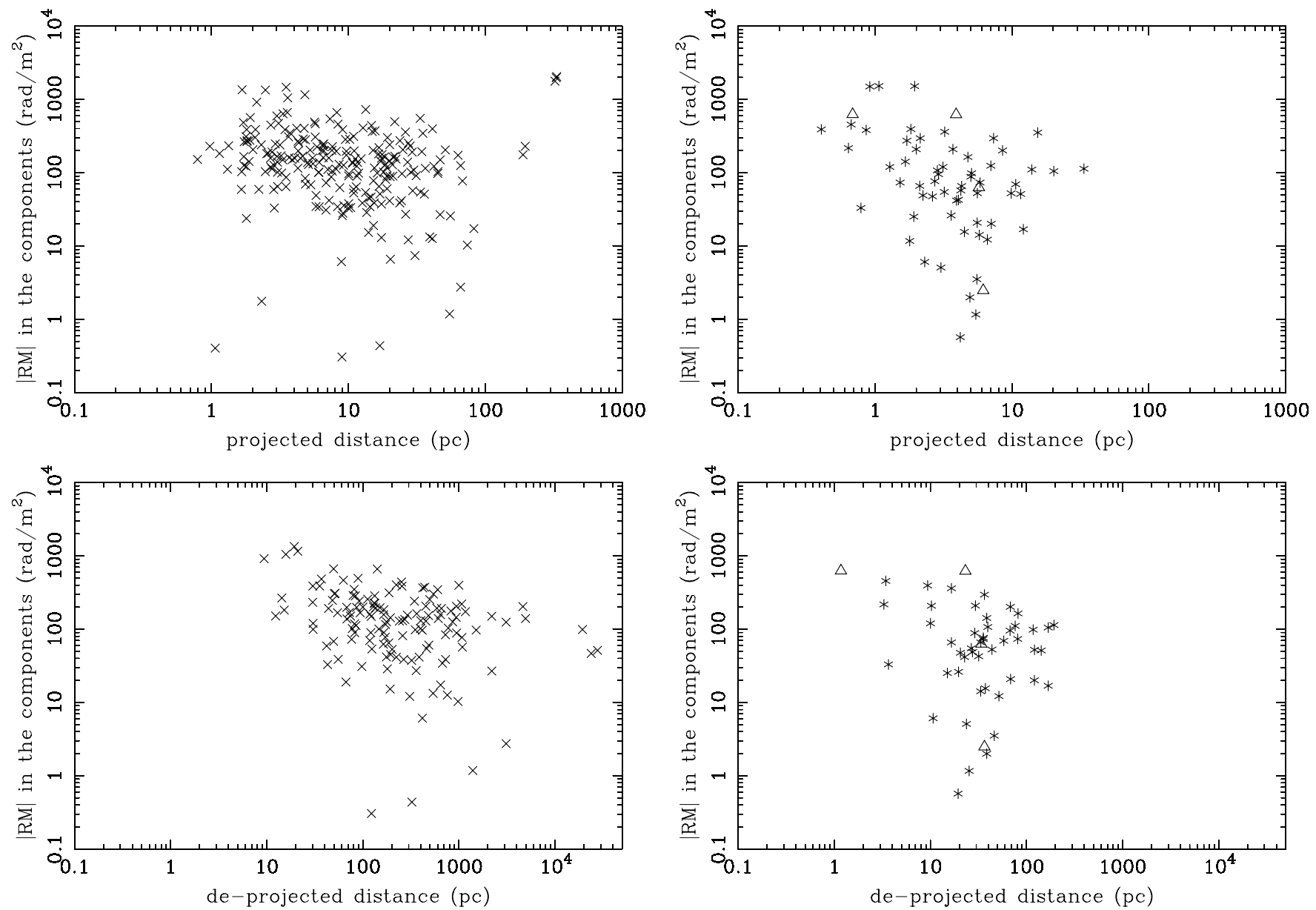

Figure 4. $\left|\mathrm{RM}_{\mathrm{obs}}\right|$ against the projected distance of the component from the core (top panel) and against the de-projected distance taking the viewing angle of the source into account (bottom panel). Left panels show quasars (crosses), and right panels show BL Lac objects (asterisks) and galaxies (triangles).

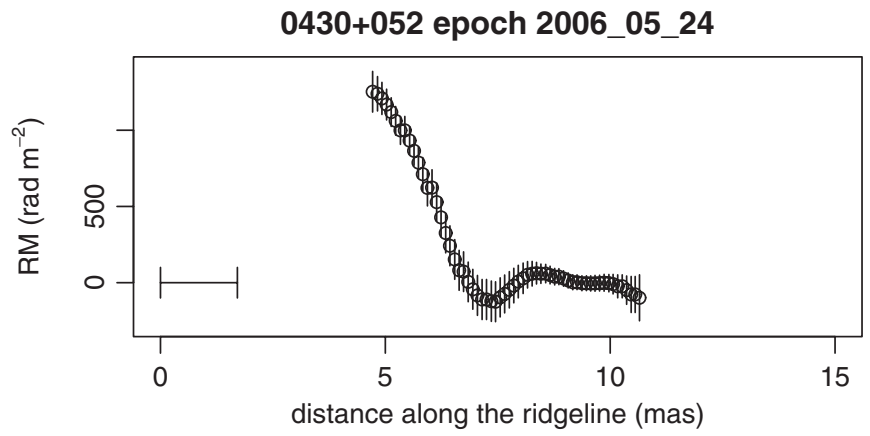

2230+114 epoch 2006_02_12

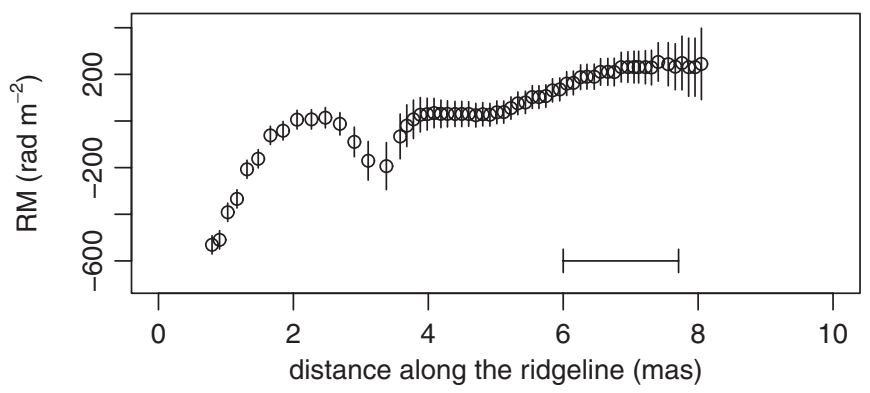

Figure 5. $\mathrm{RM}_{\mathrm{obs}}$ along the total intensity ridge line in 0430+052 (top) and $2230+114$ (bottom). The average FWHM beam size along the jet is shown in both panels as a scale bar.
However, we do not detect jet components as far away from the core in BL Lac objects as in quasars which could affect the correlation. The correlation seen in quasars supports the results from the simple core and jet component comparison in Section 3.3 showing that the amount of Faraday rotating material diminishes as a function of distance from the core.

The above approach is a simplification of a more complex behavior in the RM values as a function of distance and even if the general trend shows a decline in RM along the jet, individual sources may deviate from this trend and show complex structures. Another way to study the distance dependence is to calculate the RM values along the total intensity ridge line of the jet. Two examples are shown in Figure 5 where the top panel shows the RM along the ridge line in 0430+052 where the core is depolarized (see online figure set of Figure 1, Figure 1.28) but further along the jet, the RM declines very sharply, in accordance with the simple scenario. In 2230+114 (see online figure set of Figure 1, Figure 1.151) a more complex structure can be seen along the jet, with the RM changing sign along the jet. This example shows that with better resolution along the jet, the situation may not be as simple as a linear dependence along the jet but more complex regions are seen. The recent sensitivity upgrades of the VLBA (e.g., higher bit rate observations) will allow us to detect more polarization further down from the core and help us to study this in more sources. 
0003-066 epoch 2006_07_07

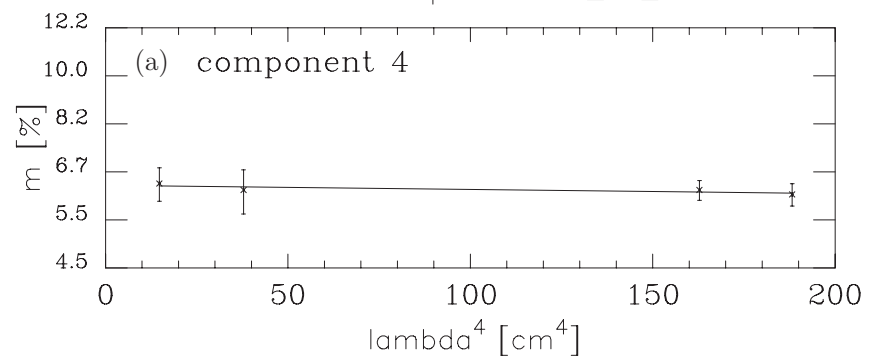

0430+052 epoch 2006_05_24

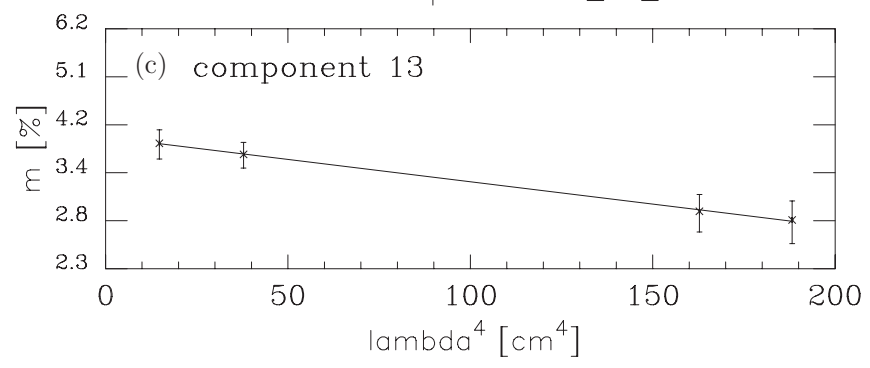

$1226+023$ epoch 2006_06_15

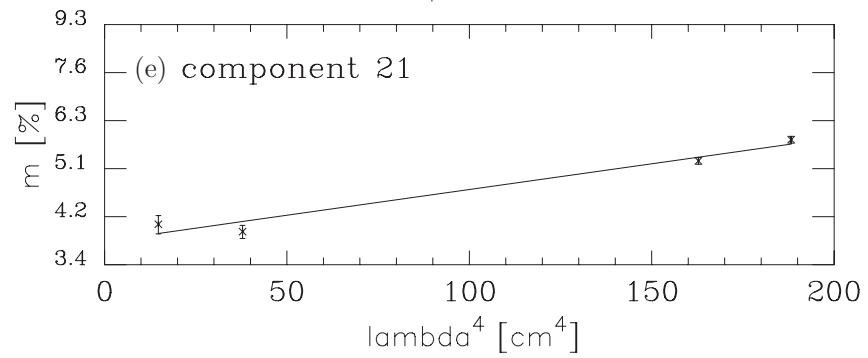

0333+321 epoch 2006_07_07

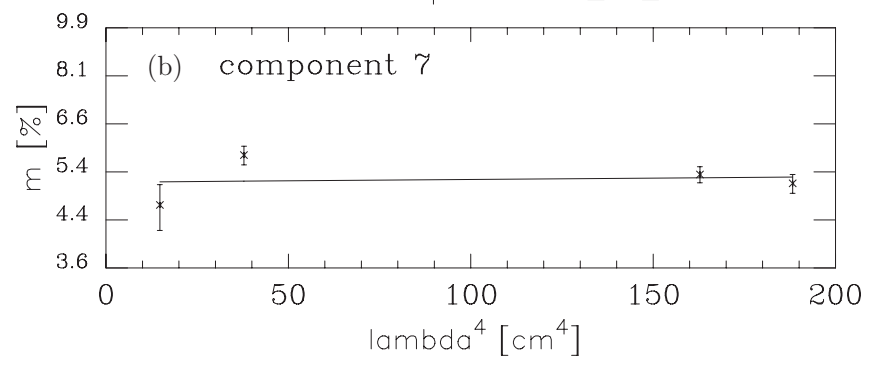

$1828+487$ epoch 2006_08_09

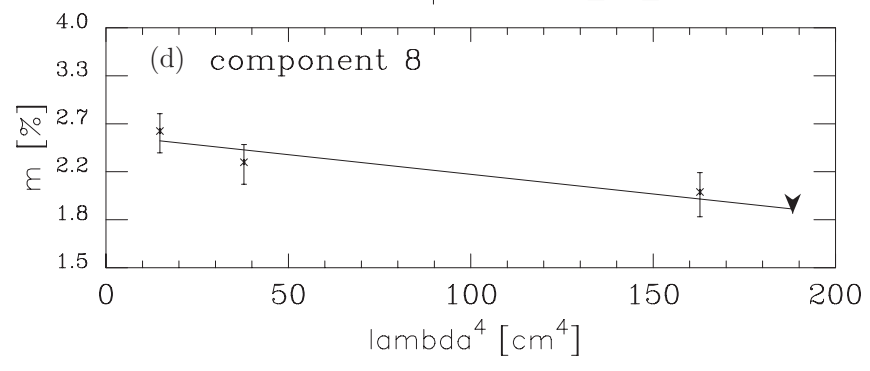

2251+158 epoch 2006_03_09

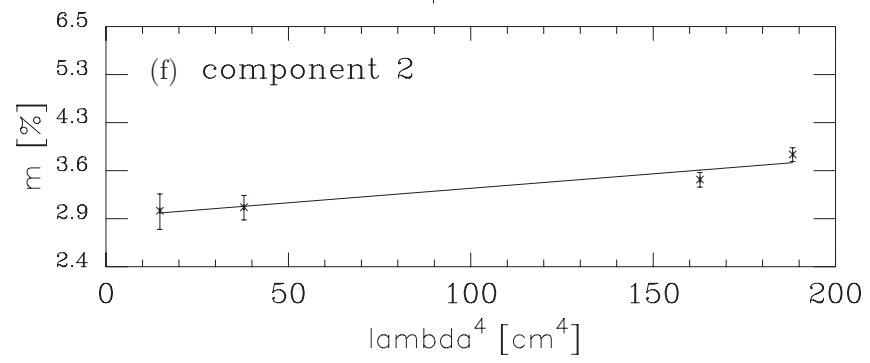

Figure 6. Examples of fits of depolarization curves to isolated components. (a) No apparent depolarization; (b) ambiguous behavior; (c) and (d) depolarization; (e) and (f) inverse depolarization. See the text for details. Upper limits are shown in arrows. Note the scale of the vertical axis which is not linear but in natural logarithm. Fits for all isolated components are available in the online journal.

(An extended version of this figure is available in the online journal.)

\subsection{Faraday Depolarization}

Faraday rotation can cause different amounts of depolarization depending on the nature of the rotating screen and if it is internal or external to the jet (Burn 1966). By applying a very simple Burn-type internal Faraday depolarization model to the core components of 40 AGNs, Zavala \& Taylor (2004) concluded that internal Faraday rotation alone cannot explain the steep decline in fractional polarization as the magnitude of the rotation measure increases. The equations they used are valid only in the optically thin regime and therefore not applicable to the core regions of AGNs at $15 \mathrm{GHz}$. We explore the viability of possible models by directly fitting individual isolated jet components for depolarization and comparing the results to our observed RM values.

For internal depolarization assuming a uniform magnetic field and the optically thin regime we have

$$
m_{\mathrm{obs}}(\%)=m_{\max }\left|\frac{\sin \left(2 \lambda^{2} \mathrm{RM}\right)}{2 \lambda^{2} \mathrm{RM}}\right|
$$

where $m_{\mathrm{obs}}$ is the observed fractional polarization, $m_{\max }$ is the maximum fractional polarization in the specific magnetic field configuration, ${ }^{13} \mathrm{RM}$ is the observed rotation measure, and $\lambda$ is the observing wavelength (Burn 1966; Homan et al. 2009). In case of external depolarization

$$
m_{\mathrm{obs}}(\%)=m_{\max } e^{-2 \sigma^{2} \lambda^{4}},
$$

where $\sigma$ is the standard deviation of the RM fluctuations and the rest of the parameters are as in Equation (3) (Burn 1966). Here we assume that the component is optically thin and homogeneous (not a combination of multiple components), and also that the angular scale of RM variations is much less than the angular resolution of our observations. The functional forms of depolarization in Equations (3) and (4) are similar over the range of $\left|\mathrm{RM}_{\mathrm{obs}}\right|$ we observe in the isolated jet components (up to $800 \mathrm{rad} \mathrm{m}^{-2}$ ) and both follow the functional form $m=m_{0} e^{b \lambda^{4}}$ where $b$ is $-2 \mathrm{RM}^{2}$ in the case of internal depolarization and $-2 \sigma^{2}$ in the case of external depolarization. We can linearize the formula to fit $\ln m=\ln m_{0}+b \lambda^{4}$ to our observations. This way we will get an estimate of total depolarization $b$ from the slope of the fit and the intercept, $\ln m_{0}$, gives the maximum polarization for that component. We use the isolated jet components only

\footnotetext{
13 Note that $m_{\max } \simeq 70 \%$ for a pure uniform magnetic field (Pacholczyk 1970); however, we do not assume a value for $m_{\max }$ in our analysis and include it as a free parameter, $m_{0}$, in our fits.
} 
Table 4

Isolated Components at $15.3 \mathrm{GHz}$ and Their Polarization Values Used in the Depolarization Analysis

\begin{tabular}{|c|c|c|c|c|c|c|c|c|c|c|c|}
\hline Source & ID & Epoch & $\begin{array}{c}r \\
\text { (mas) } \\
(4)\end{array}$ & $\begin{array}{l}\text { P.A. } \\
\text { (deg) } \\
\text { (5) }\end{array}$ & $\begin{array}{c}\mathrm{RM} \\
\left(\operatorname{rad~m}^{-2}\right) \\
(6)\end{array}$ & $\begin{array}{l}m_{15.3} \\
(\%) \\
(7)\end{array}$ & $\begin{array}{c}m_{12.1} \\
(\%) \\
(8)\end{array}$ & $\begin{array}{l}m_{8.4} \\
(\%) \\
(9)\end{array}$ & $\begin{array}{l}m_{8.1} \\
(\%) \\
(10)\end{array}$ & $\begin{array}{c}b \\
\left(\times 10^{5} m^{-4}\right) \\
(11)\end{array}$ & Significance of $b$ \\
\hline $0003-066$ & 4 & $2006 \mathrm{Jul} 7$ & 6.86 & -81.1 & $113.5 \pm 9.6$ & $8.6 \pm 1.1$ & $8.2 \pm 1.4$ & $8.2 \pm 0.6$ & $7.9 \pm 0.7$ & $-0.4 \pm 0.8$ & 0.4 \\
\hline $0119+115$ & 1 & 2006 Jun 15 & 0.09 & 7.6 & $-59.3 \pm 8.7$ & $3.2 \pm 0.1$ & $3.5 \pm 0.1$ & $3.5 \pm 0.1$ & $3.5 \pm 0.1$ & $+0.3 \pm 0.1$ & $2.0^{\mathrm{c}}$ \\
\hline $0133+476$ & 1 & 2006 Aug 9 & 2.89 & -26.6 & $-153.5 \pm 4.6$ & $10.8 \pm 0.6$ & $9.7 \pm 0.4$ & $8.4 \pm 0.4$ & $4.4 \pm 0.5$ & $-2.3 \pm 0.4$ & $5.8^{\mathrm{c}}$ \\
\hline $0149+218$ & 1 & 2006 Feb 12 & 8.60 & -11.8 & $\ldots$ & $22.7 \pm 4.5$ & $29.1 \pm 3.9$ & $15.1 \pm 3.1$ & $<10.0$ & $-4.7 \pm 1.7$ & 2.8 \\
\hline $0212+735$ & 1 & $2006 \mathrm{Jul} 7$ & 0.02 & -127.4 & $-284.9 \pm 11.6$ & $9.0 \pm 0.1$ & $8.5 \pm 0.04$ & $5.4 \pm 0.04$ & $3.6 \pm 0.1$ & $-4.1 \pm 0.1$ & $77.8^{\mathrm{c}}$ \\
\hline $0333+321$ & 7 & 2006 Jul 7 & 6.25 & 129.7 & $-1.2 \pm 8.0$ & $7.0 \pm 1.6$ & $11.7 \pm 1.1$ & $9.6 \pm 0.8$ & $8.7 \pm 0.9$ & $-1.2 \pm 0.8$ & 1.5 \\
\hline $0415+379$ & 24 & 2006 May 24 & 7.19 & 60.4 & $\ldots$ & $<11.8$ & $9.4 \pm 2.4$ & $8.0 \pm 1.9$ & $7.2 \pm 1.7$ & $-2.1 \pm 2.1$ & 1.0 \\
\hline $0429+415$ & 2 & 2006 Mar 9 & 20.54 & 59.4 & $\ldots$ & $14.0 \pm 1.0$ & $14.0 \pm 0.8$ & $2.3 \pm 0.5$ & $1.8 \pm 0.6$ & $-11.6 \pm 1.5$ & $7.8^{\mathrm{c}}$ \\
\hline $0430+052$ & 11 & 2006 May 24 & 9.49 & -117.4 & $-2.5 \pm 3.4$ & $7.5 \pm 0.5$ & $8.6 \pm 0.4$ & $7.5 \pm 0.3$ & $7.3 \pm 0.3$ & $-0.7 \pm 0.3$ & 2.1 \\
\hline $0430+052$ & 13 & 2006 May 24 & 6.00 & -114.6 & $622.0 \pm 81.5$ & $9.0 \pm 1.4$ & $8.0 \pm 1.1$ & $4.3 \pm 0.9$ & $3.9 \pm 0.9$ & $-4.9 \pm 1.4$ & 3.6 \\
\hline $0528+134$ & 2 & 2006 Oct 6 & 3.24 & 24.8 & $56.8 \pm 9.6$ & $14.7 \pm 2.4$ & $9.7 \pm 1.7$ & $8.4 \pm 1.3$ & $9.9 \pm 1.4$ & $-1.9 \pm 1.1$ & 1.7 \\
\hline $0605-085$ & 4 & 2006 Nov 10 & 2.51 & 123.6 & $41.4 \pm 9.4$ & $9.3 \pm 2.2$ & $5.5 \pm 1.7$ & $7.4 \pm 1.2$ & $7.4 \pm 1.4$ & $-0.5 \pm 1.7$ & 0.3 \\
\hline $0735+178$ & 5 & 2006 Apr 28 & 3.57 & 64.6 & $\ldots$ & $<5.0$ & $<4.0$ & $4.6 \pm 1.2$ & $5.2 \pm 1.3$ & $+0.7 \pm 2.3$ & 0.3 \\
\hline $0754+100$ & 2 & 2006 Apr 28 & 4.97 & 13.8 & $-105.1 \pm 4.5$ & $17.7 \pm 5.2$ & $22.3 \pm 3.6$ & $22.5 \pm 3.1$ & $25.4 \pm 3.3$ & $+1.0 \pm 1.3$ & 0.8 \\
\hline $0859-140$ & 2 & $2006 \mathrm{Feb} 12$ & 3.92 & 157.5 & $552.1 \pm 5.2$ & $10.1 \pm 0.9$ & $9.5 \pm 0.7$ & $9.2 \pm 0.4$ & $8.0 \pm 0.4$ & $-1.0 \pm 0.5$ & 2.0 \\
\hline $0923+392$ & 9 & $2006 \mathrm{Jul} 7$ & 1.91 & -74.6 & $\ldots$ & $1.2 \pm 0.2$ & $2.0 \pm 0.2$ & $0.5 \pm 0.2$ & $<0.6$ & $-6.3 \pm 2.1$ & $3.1^{\mathrm{c}}$ \\
\hline $1015+359$ & 8 & 2006 Mar 9 & 0.01 & -163.8 & $-159.0 \pm 9.5$ & $2.6 \pm 0.1$ & $2.5 \pm 0.1$ & $2.0 \pm 0.1$ & $1.8 \pm 0.1$ & $-1.9 \pm 0.3$ & 5.6 \\
\hline $1055+018$ & 1 & 2006 Nov 10 & 9.47 & -58.0 & $10.3 \pm 14.1$ & $16.9 \pm 4.4$ & $11.1 \pm 3.1$ & $16.3 \pm 2.7$ & $17.7 \pm 3.1$ & $+1.2 \pm 1.7$ & 0.7 \\
\hline $1148-001$ & 6 & 2006 Jul 7 & 2.41 & -121.9 & $6.6 \pm 1.7$ & $2.7 \pm 0.5$ & $3.5 \pm 0.3$ & $3.5 \pm 0.3$ & $3.8 \pm 0.3$ & $+0.7 \pm 0.7$ & 1.0 \\
\hline $1222+216$ & 4 & 2006 Apr 28 & 6.27 & 1.2 & & $12.6 \pm 3.7$ & $10.4 \pm 2.5$ & $9.8 \pm 2.1$ & $11.0 \pm 2.4$ & $-0.5 \pm 1.9$ & 0.3 \\
\hline $1226+023$ & 12 & 2006 Mar 9 & 3.89 & -136.5 & $-312.9 \pm 16.6$ & $5.1 \pm 0.1$ & $4.3 \pm 0.1$ & $2.7 \pm 0.1$ & $2.6 \pm 0.1$ & $-4.0 \pm 0.1$ & $39.4^{\mathrm{c}}$ \\
\hline $1226+023$ & 21 & 2006 Mar 9 & 5.07 & -117.3 & . & $7.9 \pm 0.5$ & $8.2 \pm 0.5$ & $9.8 \pm 0.4$ & $10.3 \pm 0.5$ & $+1.5 \pm 0.4$ & 4.0 \\
\hline $1226+023$ & 26 & 2006 Mar 9 & 12.59 & -118.2 & $193.5 \pm 11.2$ & $9.3 \pm 0.1$ & $9.3 \pm 0.1$ & $9.2 \pm 0.1$ & $8.9 \pm 0.1$ & $-0.2 \pm 0.1$ & $2.7^{\mathrm{c}}$ \\
\hline $1226+023$ & 2 & 2006 Mar 9 & 16.57 & -123.6 & $103.7 \pm 15.2$ & $4.7 \pm 1.2$ & $6.3 \pm 0.7$ & $11.2 \pm 0.4$ & $10.9 \pm 0.5$ & $+3.9 \pm 0.8$ & 4.8 \\
\hline $1226+023$ & 9 & 2006 Mar 9 & 7.27 & -129.4 & $-240.8 \pm 10.5$ & $9.5 \pm 0.2$ & $11.3 \pm 0.2$ & $11.6 \pm 0.2$ & $10.1 \pm 0.2$ & $+0.3 \pm 0.1$ & $2.7^{\mathrm{c}}$ \\
\hline $1226+023$ & 9 & 2006 Jun 15 & 7.58 & -128.7 & $-162.7 \pm 18.4$ & $9.6 \pm 0.3$ & $11.2 \pm 0.2$ & $14.2 \pm 0.2$ & $13.5 \pm 0.2$ & $+1.8 \pm 0.1$ & $13.5^{\mathrm{c}}$ \\
\hline $1226+023$ & 21 & 2006 Jun 15 & 5.20 & -117.8 & $440.6 \pm 26.9$ & $5.7 \pm 0.7$ & $5.2 \pm 0.4$ & $12.7 \pm 0.5$ & $16.6 \pm 0.7$ & $+6.9 \pm 0.5$ & 13.4 \\
\hline $1226+023$ & 2 & 2006 Jun 15 & 17.02 & -124.1 & $\ldots$ & $<4.3$ & $6.2 \pm 0.8$ & $8.2 \pm 0.5$ & $9.0 \pm 0.5$ & $+2.7 \pm 0.9$ & 3.0 \\
\hline $1226+023$ & 26 & 2006 Jun 15 & 12.70 & -118.2 & $345.4 \pm 21.6$ & $9.1 \pm 0.2$ & $8.9 \pm 0.1$ & $8.2 \pm 0.1$ & $8.0 \pm 0.1$ & $-0.7 \pm 0.1$ & 8.4 \\
\hline $1226+023$ & 12 & 2006 Jun 15 & 4.00 & -136.8 & $\ldots$ & $3.1 \pm 0.1$ & $2.9 \pm 0.04$ & $0.7 \pm 0.04$ & $0.6 \pm 0.1$ & $-9.6 \pm 0.3$ & $29.5^{\mathrm{c}}$ \\
\hline $1253-055$ & 1 & 2006 Apr 5 & 6.24 & -126.2 & $\ldots$ & $4.5 \pm 0.5$ & $3.1 \pm 0.9$ & $5.6 \pm 0.3$ & $6.0 \pm 0.3$ & $+1.8 \pm 0.8$ & 2.4 \\
\hline $1253-055$ & 5 & 2006 Apr 5 & 4.10 & -125.9 & $\ldots$ & $11.2 \pm 0.3$ & $12.7 \pm 0.5$ & $12.0 \pm 0.2$ & $10.7 \pm 0.2$ & $-0.3 \pm 0.1$ & $1.8^{\mathrm{c}}$ \\
\hline $1253-055$ & 1 & 2006 Sep 6 & 6.48 & -127.0 & $396.9 \pm 13.1$ & $6.3 \pm 0.7$ & $7.9 \pm 0.9$ & $7.0 \pm 1.9$ & $7.3 \pm 0.8$ & $+0.3 \pm 0.9$ & 0.3 \\
\hline $1253-055$ & 5 & 2006 Sep 6 & 4.32 & -125.9 & $\ldots$ & $11.6 \pm 0.4$ & $9.2 \pm 0.4$ & $5.3 \pm 0.8$ & $10.1 \pm 0.4$ & $-0.6 \pm 0.3$ & $2.3^{\mathrm{c}}$ \\
\hline $1345+125$ & 3 & 2006 Nov 10 & 44.67 & 154.8 & $\ldots$ & $13.3 \pm 3.6$ & $<8.9$ & $<6.1$ & $<7.4$ & $-3.5 \pm 2.5$ & 1.4 \\
\hline $1418+546$ & 7 & 2006 Feb 12 & 4.98 & 128.3 & $\ldots$ & $7.3 \pm 1.4$ & $9.9 \pm 1.2$ & $6.6 \pm 1.1$ & $4.3 \pm 1.3$ & $-3.0 \pm 1.4$ & 2.1 \\
\hline $1418+546$ & 7 & 2006 Nov 10 & 5.39 & 129.3 & $\ldots$ & $<7.1$ & $8.0 \pm 2.1$ & $8.9 \pm 1.9$ & $6.8 \pm 2.2$ & $+0.4 \pm 2.2$ & 0.2 \\
\hline $1458+718$ & 2 & 2006 Sep 6 & 27.27 & 166.4 & $\ldots$ & $<19.0$ & $19.9 \pm 3.2$ & $22.9 \pm 2.6$ & $26.2 \pm 3.0$ & $+1.7 \pm 1.3$ & 1.3 \\
\hline $1502+106$ & 2 & 2006 Jul 7 & 2.36 & 122.5 & $\ldots$ & $7.1 \pm 2.0$ & $5.3 \pm 1.4$ & $3.3 \pm 1.1$ & $<4.4$ & $-3.0 \pm 2.3$ & 1.3 \\
\hline $1508-055$ & 1 & 2006 Mar 9 & 17.67 & 76.8 & $\ldots$ & $<18.9$ & $<20.1$ & $24.4 \pm 4.7$ & $26.4 \pm 5.1$ & $+1.8 \pm 2.1$ & 0.9 \\
\hline $1514-241$ & 4 & 2006 Apr 28 & 4.27 & 164.9 & $\ldots$ & $<8.6$ & $8.1 \pm 1.9$ & $21.3 \pm 1.6$ & $23.7 \pm 1.6$ & $+6.5 \pm 1.5$ & 4.5 \\
\hline $1538+149$ & 4 & 2006 Jun 15 & 0.47 & -41.8 & $-26.2 \pm 12.1$ & $7.1 \pm 0.1$ & $6.9 \pm 0.1$ & $6.0 \pm 0.1$ & $6.2 \pm 0.1$ & $-0.9 \pm 0.1$ & 9.1 \\
\hline $1611+343$ & 3 & 2006 Jun 15 & 3.95 & -168.5 & $54.4 \pm 8.7$ & $7.5 \pm 1.3$ & $8.4 \pm 0.9$ & $9.8 \pm 0.8$ & $7.4 \pm 1.3$ & $+0.9 \pm 0.9$ & 1.0 \\
\hline $1611+343$ & 2 & 2006 Jun 15 & 4.30 & 149.6 & $\ldots$ & $6.6 \pm 1.2$ & $6.8 \pm 0.8$ & $7.5 \pm 0.8$ & $4.6 \pm 1.3$ & $+0.3 \pm 1.1$ & 0.3 \\
\hline $1652+398$ & 4 & 2006 Feb 12 & 8.25 & 129.9 & $\ldots$ & $23.1 \pm 5.1$ & $22.9 \pm 3.7$ & $17.6 \pm 3.8$ & $<12.9$ & $-2.6 \pm 1.8$ & 1.4 \\
\hline $1655+077$ & 2 & 2006 Nov 10 & 8.17 & -42.1 & $25.6 \pm 13.5$ & $19.2 \pm 2.0$ & $19.0 \pm 1.8$ & $13.8 \pm 1.3$ & $14.0 \pm 1.41$ & $-2.1 \pm 0.7$ & 3.0 \\
\hline $1730-130$ & 2 & 2006 Jul 7 & 8.67 & 14.1 & $-77.2 \pm 3.5$ & $9.5 \pm 1.6$ & $9.3 \pm 1.1$ & $9.3 \pm 0.9$ & $9.9 \pm 0.8$ & $+0.3 \pm 0.8$ & 0.3 \\
\hline $1730-130$ & 6 & 2006 Jul 7 & 3.08 & 26.3 & $41.6 \pm 4.6$ & $8.9 \pm 1.0$ & $7.4 \pm 0.7$ & $6.4 \pm 0.6$ & $7.4 \pm 0.5$ & $-0.8 \pm 0.6$ & 1.2 \\
\hline $1828+487$ & 8 & 2006 Aug 9 & 2.74 & -38.3 & $\ldots$ & $6.72 \pm 1.4$ & $4.8 \pm 1.0$ & $3.4 \pm 0.8$ & $<2.70$ & $-4.2 \pm 1.9$ & 2.2 \\
\hline $1828+487$ & 5 & 2006 Aug 9 & 6.29 & -27.6 & $-97.8 \pm 15.5$ & $17.1 \pm 2.1$ & $17.2 \pm 1.5$ & $12.7 \pm 1.3$ & $7.0 \pm 1.4$ & $-3.1 \pm 0.9$ & 3.6 \\
\hline $1828+487$ & 3 & 2006 Aug 9 & 11.58 & -32.5 & $-17.3 \pm 13.8$ & $20.6 \pm 2.8$ & $12.7 \pm 2.0$ & $14.4 \pm 1.7$ & $7.7 \pm 1.8$ & $-2.3 \pm 1.1$ & $2.1^{\mathrm{c}}$ \\
\hline $1908-201$ & 1 & 2006 Mar 9 & 3.49 & 37.6 & $-108.8 \pm 22.7$ & $15.8 \pm 3.3$ & $14.4 \pm 3.4$ & $12.2 \pm 2.0$ & $13.6 \pm 2.1$ & $-1.0 \pm 1.4$ & 0.7 \\
\hline $1928+738$ & 8 & 2006 Apr 28 & 2.47 & 161.2 & $19.0 \pm 13.4$ & $4.5 \pm 0.9$ & $5.9 \pm 0.5$ & $5.9 \pm 0.4$ & $5.6 \pm 0.5$ & $+0.1 \pm 0.7$ & 0.2 \\
\hline $2131-021$ & 3 & 2006 Aug 9 & 1.76 & 110.9 & $-201.9 \pm 15.7$ & $1.9 \pm 0.4$ & $2.5 \pm 0.3$ & $2.6 \pm 0.3$ & $1.8 \pm 0.4$ & $-0.0 \pm 1.0$ & 0.0 \\
\hline $2155-152$ & 1 & 2006 Dec 1 & 2.54 & -154.7 & $\ldots$ & $4.88 \pm 0.4$ & $3.4 \pm 0.3$ & $2.1 \pm 0.5$ & $3.0 \pm 0.4$ & $-2.7 \pm 0.9$ & $3.0^{\mathrm{c}}$ \\
\hline $2201+315$ & 4 & 2006 Oct 6 & 3.84 & -140.2 & $\ldots$ & $9.2 \pm 2.7$ & $<7.6$ & $8.8 \pm 1.9$ & $7.6 \pm 1.9$ & $-0.4 \pm 2.1$ & 0.2 \\
\hline $2230+114$ & 2 & 2006 Feb 12 & 11.21 & 157.6 & $\ldots$ & $<8.7$ & $12.4 \pm 2.5$ & $<5.0$ & $10.4 \pm 1.8$ & $-1.1 \pm 1.7$ & 0.6 \\
\hline $2251+158$ & 2 & 2006 Mar 9 & 6.08 & -80.1 & $-149.8 \pm 7.6$ & $3.5 \pm 0.4$ & $3.6 \pm 0.3$ & $4.3 \pm 0.2$ & $5.1 \pm 0.2$ & $+2.1 \pm 0.5$ & 4.0 \\
\hline $2251+158$ & 1 & 2006 Mar 9 & 8.57 & -64.9 & $2.8 \pm 4.6$ & $11.7 \pm 0.9$ & $12.8 \pm 0.6$ & $14.5 \pm 0.4$ & $15.2 \pm 0.4$ & $+1.2 \pm 0.3$ & 4.1 \\
\hline $2251+158$ & 2 & 2006 Jun 15 & 6.07 & -79.9 & $26.8 \pm 37.5$ & $2.7 \pm 0.4$ & $4.2 \pm 0.3$ & $4.1 \pm 0.2$ & $3.3 \pm 0.3$ & $+0.1 \pm 0.6$ & $0.2^{\mathrm{c}}$ \\
\hline $2251+158$ & 1 & 2006 Jun 15 & 8.57 & -64.0 & $-124.9 \pm 8.0$ & $16.4 \pm 1.0$ & $14.5 \pm 0.7$ & $16.4 \pm 0.5$ & $12.3 \pm 0.7$ & $-0.1 \pm 0.3$ & $0.4^{\mathrm{c}}$ \\
\hline
\end{tabular}

Notes. Columns are as follows: (1) IAU Name (B1950.0); (2) ID of the component $(0=$ core); (3) epoch; (4) component distance from the phase center of the $I$ map; (5) position angle of the component from the phase center; (6) $\mathrm{RM}_{\mathrm{obs}}$ of the component (7) fractional polarization at $15.3 \mathrm{GHz}$. Upper limits are $3 \sigma$ limits in fractional polarization; (8) fractional polarization at $12.1 \mathrm{GHz}$; (9) fractional polarization at $8.4 \mathrm{GHz}$; (10) fractional polarization at $8.1 \mathrm{GHz}$; (11) slope b from the depolarization fit in Section 4.2; (12) significance of the slope. Sources marked with $\mathrm{c}$ have a $\chi^{2}>6$, indicating low probability that our simple model is accurate in explaining how the polarization changes with wavelength. 

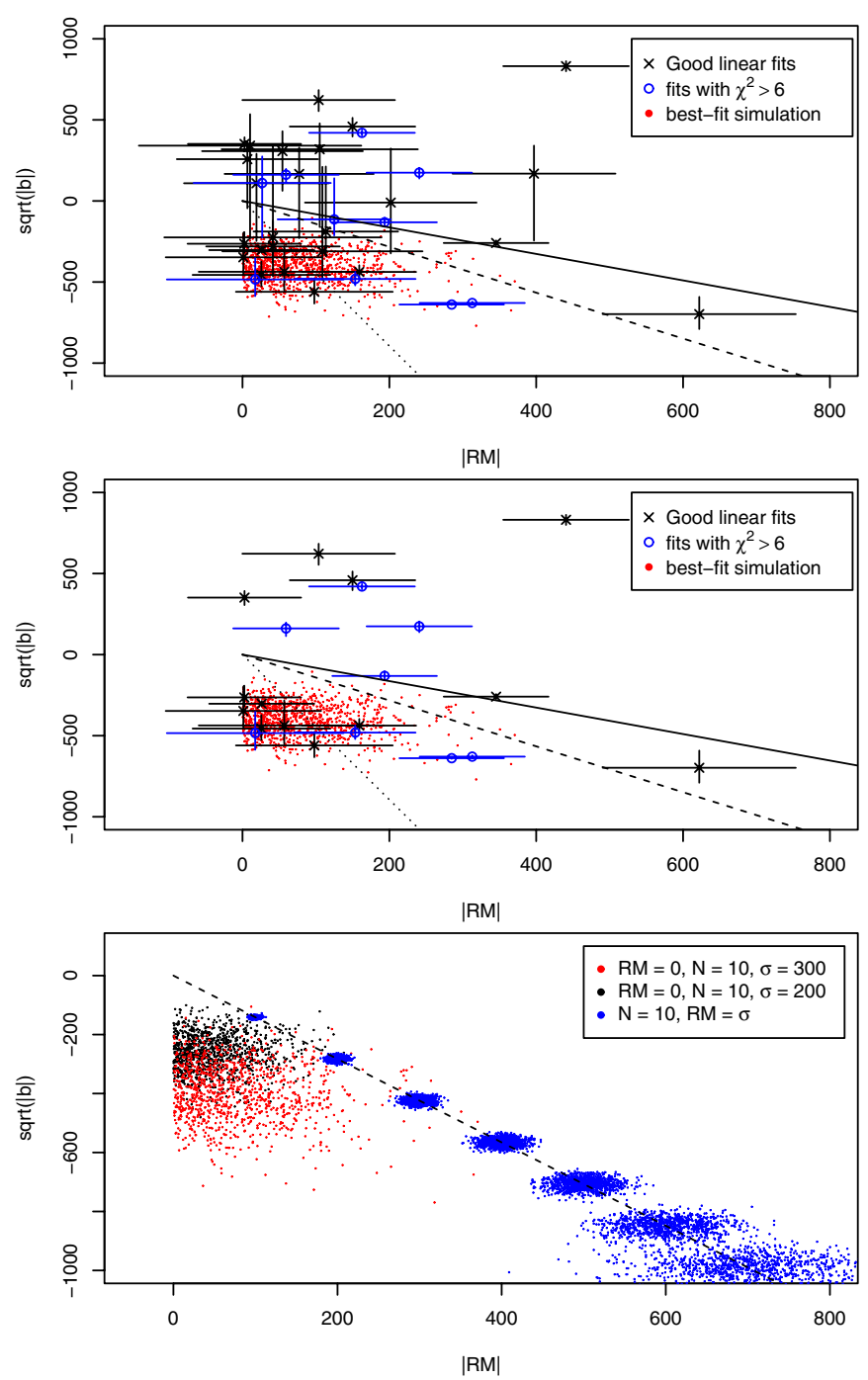

Figure 7. Top: fitted slope (b) from Figure 6 against the $\left|R M_{\mathrm{obs}}\right|$ in the isolated jet components. Crosses have good fits with $\chi^{2}<6$, open circles (blue in the online journal) have $\chi^{2}>6$ and may not be well described by our simple model. Gray dots (red in the online journal) are our best-fit simulation case from the bottom panel. The solid line shows the expected amount of internal Faraday depolarization and dashed and dotted lines the expected amount of external Faraday depolarization for different parameters (see the text for details). Middle: same as top panel but showing only components that are more than $2 \sigma$ from $b=0$. Bottom: simulations for external depolarization over varying average RM, number of lines of sight $N$ and dispersion $\sigma$ in the RM. The dashed line shows the expected amount of external Faraday depolarization when average $\mathrm{RM}=\sigma$. Dark gray (blue in the online journal) dots show the same from several simulations using $N=1000$. Black dots have average $\mathrm{RM}=0, N=10$, $\sigma=200$; light gray (red in the online journal) dots have average $\mathrm{RM}=0$, $N=10, \sigma=300$.

(A color version of this figure is available in the online journal.)

to ensure that we are looking at homogeneous components in the optically thin part of the jet. The polarization values for the isolated components are given in Table 4 where the RM is given in column (6), columns (7)-(10) show the fractional polarization and its error at the different frequency bands, and column (11) the value $b$. In Figure 6 we show examples of the fits and in the top panel of Figure 7 we show the square root of $|b|$ with its sign preserved to distinguish depolarization from inverse depolarization where polarization is higher at $8 \mathrm{GHz}$ than at $15 \mathrm{GHz}$. In 16 out of 61 components our simple model does not fit the data well, which may be an indication of a more complex behavior than the simple exponential model can explain. These components are clearly marked in Figure 7 and Table 4.

The solid line in the top panel of Figure 7 gives the expected amount of internal Faraday depolarization for a given RM. If internal Faraday depolarization alone would account for our observations, most of the data points should fall on this line, which is not the case. The majority of the depolarized components fall below the line, indicating that the fractional polarization in these components falls faster than expected for internal Faraday rotation. This is clearer in the middle panel of Figure 7, which shows only the components that are more than $2 \sigma$ away from $b=0$. The same appears to be true for external depolarization when we assume that the dispersion in the RM values is proportional to the observed $\mathrm{RM}$, i.e., $\sigma^{2}=\mathrm{RM}^{2}$ (dashed line). Most of the components fall below this line, indicating that the dispersion in the Faraday screen is larger than the mean RM produced by that screen. This suggests that the Faraday screen is dominated by random RM fluctuations between independent lines of sight. For a random Faraday screen the observed average RM will approximately follow a relation $\mathrm{RM} \propto \sigma / \sqrt{N}$ where $N$ is the number of lines of sight. The dotted line in the top panel of Figure 7 uses $N=10$ for the calculation of $\sigma$ in Equation (4), where we assume the angular scale of the RM dispersion to be much smaller than the beam so that $\sigma^{2}=10 \mathrm{RM}^{2}$. This line fits our data much better than assuming $\sigma^{2}=\mathrm{RM}^{2}$, but there is still a large scatter about the line. The line produced by Equation (4) assumes the scale of Faraday rotating cells to be much smaller than the beam size. This may not be true for high-angular-resolution observations such as those by the VLBA (Tribble 1991). In order to take the number of lines of sight correctly into account, we directly simulate the expected depolarization and RM for a variety of $\sigma$, $\mathrm{RM}$, and $N$ combinations.

In the simulations, we define $\lambda^{2}$ values in the range of 0 to $1.6 \times 10^{-3} \mathrm{~m}^{2}$ and initialize each frequency to have $70 \%$ fractional polarization and $0^{\circ}$ EVPA. ${ }^{14} \mathrm{We}$ pass this initial polarization through $N$ individual lines of sight. Each individual line of sight is simulated by adding an average RM, which is the same for all lines of sight, and a random contribution drawn from the Gaussian distribution of variance $\sigma^{2}$. For each wavelength, we sum the contribution of $N$ lines of sight drawn in a similar manner to obtain an average $p$ and EVPA value. We treat these average values as our real observations and fit the $p$ values for $b$ and EVPA values for RM. We repeated the simulation 1000 times to obtain a range of $b$ and RM values to compare with our observations. In Figure 7 bottom panel we plot several of our simulations, and in the top panel we show our best-fit case overlaid with the real data points.

The blue dots in the bottom panel of Figure 7 are from several simulations with a large number of lines of sight $(N=1000)$ where we have set average $\mathrm{RM}=\sigma$ (using values 100, 200, 300, 400, 500, 600, and 700 for RM and $\sigma$ ), and these simulations are plotted with the dashed line produced by Equation (4). As can be seen, these large $N$ simulations follow the expected curve very well with increased scatter for the large $\sigma$ values. As described above, our data in the top panel of Figure 7 largely fall below this line, indicating that the Faraday screens may be random screens with a small number of lines of sight. To test this possibility, we set the average RM applied to all lines of sight to be 0 in our simulation, the number of lines of sight $N=10$, and

\footnotetext{
14 Note that the initial values chosen here do not affect our results as we are interested only in the change in $p$ and EVPA due to combination of multiple lines of sight within our beam.
} 
either $\sigma=200 \mathrm{rad} \mathrm{m}^{-2}$ (black dots) or $\sigma=300 \mathrm{rad} \mathrm{m}^{-2}$ (red dots). The red dots cover almost the same region as our data while the black dots produce too little depolarization. As one might expect for a random-walk style Faraday screen, $\sigma / \sqrt{N}$ for the red dots is $95 \mathrm{rad} \mathrm{m}^{-2}$, which is in close agreement with the observed median $|\mathrm{RM}|=104 \mathrm{rad} \mathrm{m}^{-2}$ for our sample of isolated components. Therefore, we conclude that most of our observations can be explained with a completely random external foreground screen viewed through a small number of lines of sight. This implies that the linear size of the RM cells may not be too much smaller than our beam size. We plot the red dots of the bottom panel of Figure 7 in the top panel to show the good correspondence with our data.

Additional complications to note are that if the depolarization is much higher, we do not detect enough polarization at $8 \mathrm{GHz}$ to calculate the RM or the internal rotation causes non- $\lambda^{2}$ law behavior and we do not get a good enough fit in our RM calculation. To study this further, we have included in Table 4 isolated jet components for which we detect fractional polarization at some of our four frequency bands but not necessarily all (15 components), and 10 components for which we detect fractional polarization but no RM (a sign of non- $\lambda^{2}$ behavior). In the calculation of the slope $b$, we have assumed the upper limits to be detections. This way we get a lower limit estimate for the depolarization.

Based on the polarization behavior in Figure 7, we can divide all our fits into four categories. Constant polarization over the frequency range is seen in 13 out of 60 components, as the example case in Figure 6(a). These components are within error bars of zero $b$ in Figure 7. In 18 components, the fractional polarization did not follow a linear trend but was changing randomly between the frequencies, as seen in Figure 6(b). In several of these the slope $b$ is consistent with zero within the error bars. Depolarization is seen in 20 of the components, and two examples are given in Figures 6(c) and (d). In 1828+487 we were not able to calculate an RM value because we only detect an upper limit in linear polarization at the $8.1 \mathrm{GHz}$ band. From the slope of the fit we can estimate that the amount of internal Faraday rotation required to cause such depolarization would be $970 \mathrm{rad} \mathrm{m}^{-2}$, higher than what we observe in any of the isolated components. There are four additional components which show slopes steeper than the typical range in Figure 7, and the depolarization in these sources could be produced by internal Faraday depolarization.

Additionally, we see nine components with inverse depolarization structure, where the fractional polarization at $8 \mathrm{GHz}$ is higher than at $15 \mathrm{GHz}$. In only five of these we detect RM as well and these are the most significant points above zero $b$ in Figure 7. The other inverse depolarization components above zero are in the category where a linear fit did not describe the fractional polarization behavior well and the slope $b$ is not a good indicator of the depolarization. The nine components each show a significant rise in the fractional polarization as shown in Figures 6(e) and (f). This is unexpected and cannot be easily explained with any standard external depolarization models. Interestingly, seven of the nine components (and all the five for which we have RM value) are in 3C 273 and in 3C 454.3, both of which show transverse RM gradients in their jets (see Section 4.3). The other two are in $1458+718$ where the slope is still within $2 \sigma$ from zero, and in 1514-241 where the fractional polarization rises from an $8.6 \%$ upper limit at $15 \mathrm{GHz}$ to $24 \%$ at $8 \mathrm{GHz}$. In this source we do not detect any RM values. Internal Faraday rotation together with helical or loosely tangled ran- dom magnetic field configurations could possibly explain the observed inverse depolarization and this model is investigated in detail by Homan (2012).

\subsection{Transverse RM Gradients}

If AGN jets are launched from a rotating black hole or accretion disk, it could be expected that the magnetic field around the jet has an ordered toroidal component (e.g., McKinney $\&$ Narayan 2007). A signature of such a toroidal component (often interpreted as a component of a helical field) would be a rotation measure gradient transverse to the jet flow direction as the line-of-sight magnetic field changes its direction (e.g., Blandford 1993). In this case, the gradient should be seen in multiple locations of the jet, which distinguishes it from isolated local gradients that arise from changes in the density of the Faraday rotating material. The detection of such gradients is challenging due to the limited number of bright sources with polarized, well-resolved jets (Taylor \& Zavala 2010). Furthermore, the jet structures can be very complex, and it is likely that both kind of gradients exist in the same sources, as in the case of the radio galaxy 3C 120 (Gómez et al. 2011). Therefore, even if a transverse RM gradient is observed, it does not automatically indicate the presence of a helical magnetic field, and detailed modeling is needed to probe its nature.

In Appendix C.2, we perform simulations to investigate how large spurious transverse gradients can arise due to image noise and finite restoring beam size. Based on our simulations we conclude that the convolved jet should be at least 1.5 beams wide (but preferably more than 2) in polarization along the direction of the gradient and that a gradient should exceed the $3 \sigma$ level to be considered significant. We define $\sigma$ as the largest RM error at the edge of the jet when the systematic error due to absolute EVPA calibration, $\sim 60 \mathrm{rad} \mathrm{m}^{-2}$, is first removed in quadrature. The significance of a gradient is then simply the total change in RM divided by the $\sigma$. These criteria are similar to the ones described by Broderick \& McKinney (2010) and Taylor \& Zavala (2010), although our simulations indicate a minimum transverse width of 1.5-2 rather than three beam widths. Broderick \& McKinney (2010) show that due to the complexity of AGN cores, gradients within one beam width of the core may be unreliable at our resolution, and therefore we have not considered these regions in our study. Murphy \& Gabuzda (2012) argue that an RM gradient due to helical magnetic field is significant even when the jet is not resolved, based on simulations where they convolve a simulated gradient with different beam sizes. However, their simulation does not take into account that a spurious gradient can arise due to noise in the data, which we show to be a major effect on VLBA observations of unresolved jets.

Following the above guidelines, we examined all our RM maps in detail. Our observations show a clear gradient across the jet of 3C 273 (Figure 8), confirming the observations of Asada et al. (2002, 2008a) and Zavala \& Taylor (2005). The gradient is detected above the $3 \sigma$ level, and the jet is nearly three beams wide along the gradient direction. For the first time the RM is seen to change sign over the gradient, which is a further indication of a helical field. We believe we are seeing this now due to a different part of the jet being illuminated in the earlier observations, similarly as seen in 3C 120 by Gómez et al. (2011). In fact, if we compare the mean jet direction, calculated from model-fit components within 7 mas from the core, in our 2006 observations to the 2000 observations by Zavala \& Taylor (2005), we see a change of $10^{\circ}$. We also do not detect as high positive RM values as Zavala \& Taylor (2005), who see 

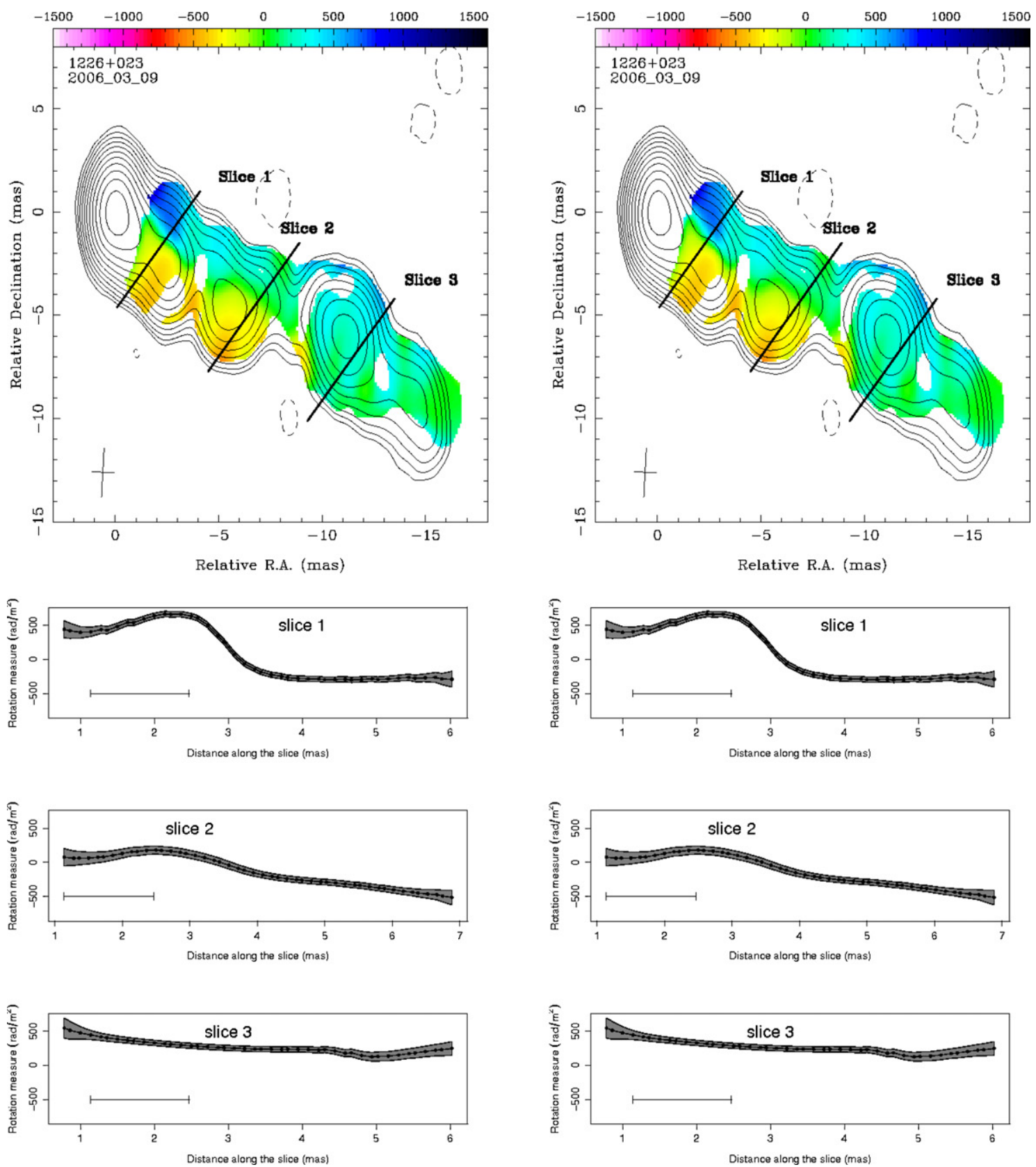

Figure 8. Rotation measure maps of 3C 273 with high-RM pixels in the cores blanked for our March (left) and June (right) epochs. The black lines show slices transverse to the jet from which the gradients (bottom three panels) have been taken. The beam size along the slice is shown in each plot with a scale bar.

(A color version of this figure is available in the online journal.)

values up to $2000 \mathrm{rad} \mathrm{m}^{-2}$, while our maximum values are near $500 \mathrm{rad} \mathrm{m}^{-2}$. The maximum gradient is detected about 3-7 mas from the core, where the $\mathrm{RM}$ changes from $+500 \mathrm{rad} \mathrm{m}^{-2}$ to $-600 \mathrm{rad} \mathrm{m}^{-2}$. Further down the jet the gradient becomes less pronounced and we also detect less polarized jet emission.

Asada et al. (2002) suggested that the gradient originates from a helical magnetic field in the jet. Based on the large RM values observed in their study, Zavala \& Taylor (2005) preferred an external origin, possibly a sheath around the jet. The main argument they used was that internal Faraday rotation values of $2000 \mathrm{rad} \mathrm{m}^{-2}$ should cause severe depolarization in a uniform magnetic field, which was not observed. However, combinations of different magnetic field configuration and number of lines of sight can possibly explain high RM and complex polarization structure (see Section 4.2). Asada et al. (2008a) report variations in the transverse gradient of 3C 273 between their observations in 1995 and 2002. They present several calculations ruling out the narrow-line region as the origin of the Faraday rotation due to the variability and suggest that the variations are caused by the external slower moving sheath changing over a timescale of several years. Our observations are only three months apart, but there are still differences between the maps, especially in the region 2-5 mas from the core on the south side of the jet as can be seen in the values of slice 1 . In the first epoch the $\mathrm{RM}$ changes from +450 to $-300 \mathrm{rad} \mathrm{m}^{-2}$ but in our second epoch the change is from +500 to $-760 \mathrm{rad} \mathrm{m}^{-2}$. The large discrepancy in the negative values is also seen when examining component 14 in Table 3, located 2.7 mas from the core in Figure 8 . The component has not moved more than 0.2 mas $(0.6 \mathrm{pc})$ over the two epochs, and still the values differ by over 
$400 \mathrm{rad} \mathrm{m}^{-2}$ so the change cannot be caused by the component illuminating a different part of the Faraday screen. If the scale of RM variations in the screen were this small, we would not expect to see consistent RM values over the jet or well-defined gradients.

This component also shows clear depolarization between 15 and $8.1 \mathrm{GHz}$ as the fractional polarization drops from $9.2 \%$ to $2.8 \%$ in the first epoch and from $8.4 \%$ to $1.4 \%$ in the second. This component is not on our list of isolated jet components due to the proximity of the brighter (in total intensity) component 12 and is therefore not shown in Figure 7. However, if we fit the polarization data similarly as in Section 4.2, we obtain values $-\sqrt{|b|}$ of -843 and -1001 for the first and second epoch, respectively. This would make the component the most depolarized in our sample. It is very difficult to explain such fast variations with external Faraday rotation, and therefore it is possible that we are seeing internal Faraday rotation in this case. Another alternative is that the variations we observe in the RM are due to interaction of the jet with a sheath. Chen (2005) observed variations of comparable magnitude over similar timescales in $3 \mathrm{C} 273$. He proposes that the fast variations could be caused by expansion of the components compressing the surrounding medium increasing the magnetic field and electron density. However, it is difficult to explain the complex depolarization observations with this model.

The observational signatures of large-scale helical magnetic fields were recently studied from a theoretical perspective by Clausen-Brown et al. (2011). They suggest that the best way to distinguish signatures of helical fields from interaction with an external medium is to look for correlated behavior in the total intensity, spectral index, and polarization profiles. In their model, the total intensity, polarization, and spectral index should have skewed profiles, so that a tail in total intensity is found on the same side of the jet where the polarization is lower and where the spectral index is steeper. The skewness of the profiles depends on the Lorentz factor and viewing angle of the jet. We have studied the total intensity, polarization, and spectral index profiles at the locations of the gradients in 3C 273 and show them for slice 2 in our 2006 March epoch in Figure 9. It is obvious that the profiles are skewed in the way predicted by Clausen-Brown et al. (2011), supporting models with helical magnetic fields in 3C 273. The spectral index gradient was also detected by Savolainen et al. (2008). Unfortunately, such skewed signatures are in general difficult to detect due to beam effects and large errors in polarization toward the jet edges, and even in our observations the signature is not as clear in all jet locations. Our higher resolution and more sensitive VLBA follow-up observations will enable us to study this further.

Another source which shows a significant transverse gradient in its 2006 March epoch is 3C 454.3, shown in Figure 10. The gradient is seen between 1 and 3 mas from the core and exceeds $3 \sigma$. The magnitude of the gradient varies slightly depending on the chosen location with a maximum of about $63 \mathrm{rad} \mathrm{m}^{-2} \mathrm{mas}^{-1}$. In the slice of Figure 10 it is about $57 \mathrm{rad} \mathrm{m}^{-2} \mathrm{mas}^{-1}$ when the jet is three beams wide. In our second epoch in 2006 June, the gradient is not as clear, but that can be attributed to lower data quality in the $8.1 \mathrm{GHz}$ band during that epoch, as a smaller region of the jet is visible above the noise level. Another complication arises from the bending of the jet because it is difficult to determine the transverse direction when the jet bends. In 3C 454.3 we have chosen the local jet direction when studying the gradient, but it is obvious that the gradient is no longer seen further down in the jet after it bends. We see variations in the
RMs of the jet components 1 and 2, which are 8.6 and 6.1 mas from the core, respectively, as well as inverse depolarization in several components, and this could point toward internal Faraday rotation as seen in 3C 273. Interestingly, Figure 27 in Zavala \& Taylor (2003) seems to hint at a RM gradient in the same direction, although this was not reported by Zavala $\&$ Taylor (2003), who were not concerned with the possible presence of transverse gradients in their RM maps. When our observations are combined with total intensity, polarization and spectral index observations (Zamaninasab et al. 2012, in preparation) they seem to follow a modification of the ClausenBrown et al. (2011) model. Details of this modeling will be presented in Zamaninasab et al.

Additionally, several other sources show interesting transverse RM structures. In $2230+114$ we detect a gradient of $144 \mathrm{rad} \mathrm{m}^{-2} \mathrm{mas}^{-1}$ at $3 \sigma$ level about 7 mas from the core where the jet is 1.9 beams wide (Figure 11). Based on our criteria above, this can be considered as a significant gradient, but it is more difficult to tie it to any specific model because the region over which the gradient is detected is small. Therefore, we have included the source in follow-up VLBA observations which are designed to give better sensitivity and resolution in hope of confirming the gradient and modeling it in more detail. The results of the follow-up observations will be presented in a separate paper.

Similarly, 0923+392 shows a significant total gradient of over $624 \mathrm{rad} \mathrm{m}^{-2}$ where the polarized jet is 2.6 beams wide (Figure 11). The gradient, however, is confined within one beam width and is about $385 \mathrm{rad} \mathrm{m}^{-2}$ mas $^{-1}$ and depends on the high $\mathrm{RM}$ region at the northern side of the jet. Interestingly, the high $\mathrm{RM}$ values are seen right where the jet is shown to bend outside the line of sight of the observer (Alberdi et al. 2000) and therefore could be a sign of the jet interacting with the intergalactic medium. The polarization structure we observe is also consistent with the observations of Alberdi et al. (2000) where the change in polarization over several years is shown to be consistent with a moving component interacting with a stationary feature where the jet bends. Another alternative could be that the three-dimensional geometry of the jet is complex and the north and south sides of the jet probe different regions along the jet so that the other is further downstream and we are seeing effects of different optical thickness. With the present data it is difficult to distinguish between the alternative models. Therefore, this source was also included in our follow-up VLBA observations.

We do see hints of transverse gradients in three other sources as well, but none of these fulfill all of our criteria. For example, in 2134+004 (see online figure set of Figure 1, Figure 1.139) the change in the RM is more than $2 \sigma$, but it is detected only at a single location of the jet and is only $65 \mathrm{rad} \mathrm{m}^{-2} \mathrm{mas}^{-1}$ where the jet is 1.8 beams wide, so we do not call it significant. In $0945+408$ the RM map (see online figure set of Figure 1, Figure 1.67) clearly shows two different RM regions, and even though the jet is more than two beams wide, the gradient is within $2 \sigma$ errors. A similar gradient is seen in $1641+399$ (see online figure set of Figure 1, Figure 1.110) where it also is within $2 \sigma$ errors. Therefore, we cannot call the gradients in these sources significant but have included them in the followup higher sensitivity observations for further study.

We have also compared our maps to other studies reporting transverse RM gradients in the sources in our sample. Asada et al. (2008b) and Reichstein \& Gabuzda (2011) report a transverse gradient of a few hundred $\mathrm{rad} \mathrm{m}^{-2}$ in the source 

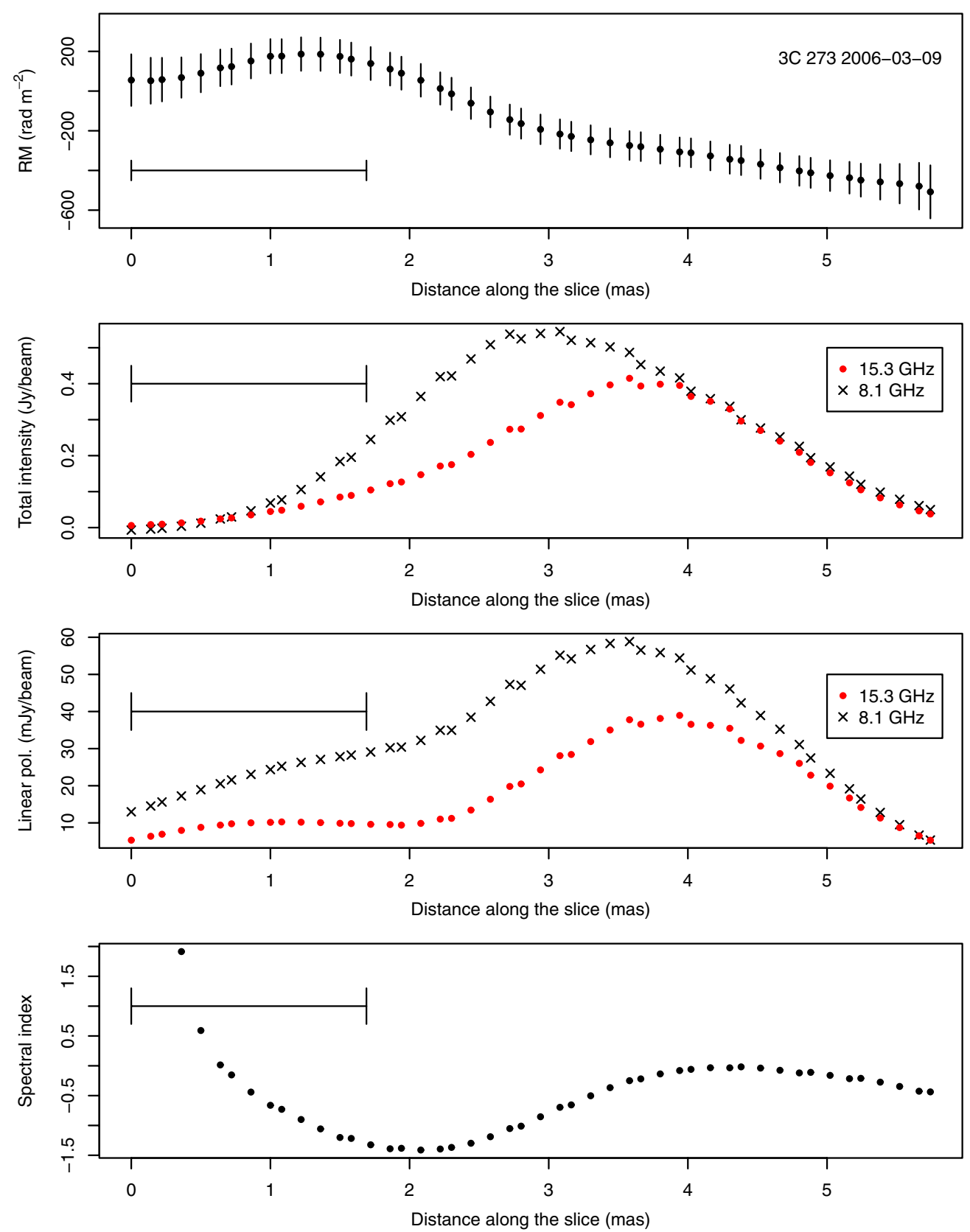

Figure 9. Transverse slices of 3C 273 along slice 2 in Figure 8 left panel for RM (top panel), total intensity (second panel from the top), linearly polarized intensity (third panel from the top), and spectral index between 15.3 and $8.1 \mathrm{GHz}$ (bottom panel). $15.3 \mathrm{GHz}$ observations are shown in (red) filled circles and $8.1 \mathrm{GHz}$ observations in (black) crosses. The rms error in total intensity is $1.2 \mathrm{mJy}_{\text {beam }}^{-1}$ and $4.0 \mathrm{mJy} \mathrm{beam}^{-1}$ at 15.3 and $8.1 \mathrm{GHz}$, respectively. The rms error in linear polarization is 0.6 and $0.8 \mathrm{mJy}_{\text {beam }}{ }^{-1}$ at 15.3 and $8.1 \mathrm{GHz}$, respectively. The error in spectral index varies from 0.004 in the center of the jet to 0.3 at the edges. The beam size along the slice is shown in each plot with a scale bar.

(A color version of this figure is available in the online journal.)

$0333+321$ in VLBA observations between 5 and $8 \mathrm{GHz}$. We do see a gradient of similar magnitude (see online figure set of Figure 1, Figure 1.21), but it extends only one beam width across the jet, and it is also very much dependent on low-S/N-jet edge pixels. Additionally, it does not extend over the whole length of the jet. Some of this may be due to our higher observing frequency, which causes us to detect less polarized emission across the jet. Therefore, we do not consider this a robust gradient in our images but merely suggestive of a possible gradient. Reichstein \& Gabuzda (2011) also report gradients in both the core and jet of $1150+812$ but in both cases the slices they take are less than two beams wide at the location of the gradient. We do see a change in RM values in a similar direction (see online figure set of Figure 1, Figure 1.79), but again it is very much dependent on unreliable edge pixels and also the jet is only one beam width across at our $8 \mathrm{GHz}$ resolution so that we do not consider the gradient to be robust.

In 3C 120 (see online figure set of Figure 1, Figure 1.28) we do not detect the transverse RM gradient that was seen by Gómez et al. (2008) in observations made in 2001 at 15, 22, and $43 \mathrm{GHz}$. This is not unexpected because Gómez et al. (2011) demonstrate in their Figure 9 how a different region of the jet in 3C 120 is seen in their 2001 and 2007 observations at $15 \mathrm{GHz}$. Our observations are close to their latter epoch, and therefore we also see a different part of the jet and do not detect the gradient. 

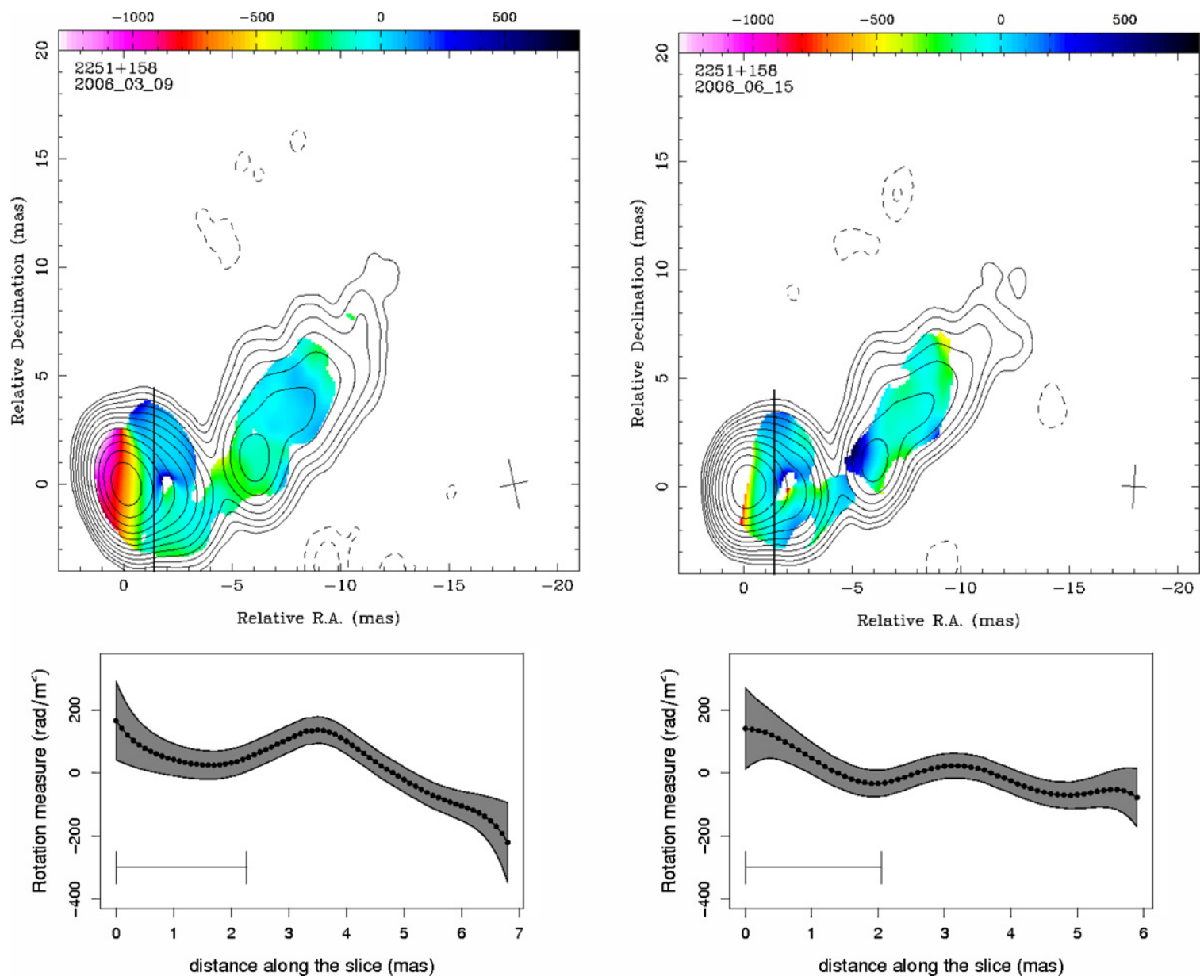

Figure 10. Rotation measure maps of 3C 454.3 with high-RM pixels blanked for our March (left) and June (right) epochs. The beam size along the slice is shown in each plot with a scale bar.

(A color version of this figure is available in the online journal.)

Other reported sources include $0836+710$, for which Asada et al. (2010) find a possible gradient of $\sim 150 \mathrm{rad} \mathrm{m}^{-2}$ in observations between 4.6 and $8.6 \mathrm{GHz}$. The gradient is at a location in which we were not able to obtain good $\lambda^{2}$-fits (see online figure set of Figure 1, Figure 1.59) and therefore we cannot confirm the result. In any case, the jet is not transversely well resolved in our observations. Another source of interest is 1803+784, for which Mahmud et al. (2009) report RM gradients in four epochs observed with several different frequency setups from 4.6 to $43 \mathrm{GHz}$. In our map we do not see any indication of a gradient (see online figure set of Figure 1, Figure 1.122), but it must be noted that our map does not extend as far down the jet as some of theirs. This is because in their lower frequency maps they detect polarization further down the jet compared to our $15 \mathrm{GHz}$ observations. O'Sullivan \& Gabuzda (2009) studied the Faraday rotation in six blazars observed in 2006 July between 4.6 and $43 \mathrm{GHz}$, and detected a transverse gradient in two of them in multiple frequency ranges. In 0954+658 they detect a gradient which is mainly visible in the low-frequency RM map from 4.6 to $15.4 \mathrm{GHz}$. Even in this case the jet is unresolved. In the 7.9-15.4 GHz RM map, very close to our resolution, they see an indication of the gradient, but again the jet is unresolved. Our map (see online figure set of Figure 1, Figure 1.69) shows similar changes in the RM from positive to near $0 \mathrm{rad} \mathrm{m}^{-2}$ at the same location, but the jet is less than two beam widths wide and the gradient is also very much dependent on the edge pixels, which we have noted in the previous section to be unreliable. We cannot compare the core RM values because we do not find good $\lambda^{2}$-fits in the core region. We also detect changes in the jet RM because in our observations the jet RM varies between 0 and $600 \mathrm{rad} \mathrm{m}^{-2}$ while in the same frequency range map in O'Sullivan \& Gabuzda (2009) the values are between 0 and $200 \mathrm{rad} \mathrm{m}^{-2}$. This means that we are either seeing a different part of the jet, or the RM has changed over a time span of three months. The gradient they detect in $1156+295$ is highly dependent on a few unreliable edge pixels. Additionally, it is within one beam width from the core and therefore unreliable as shown by the simulations of Broderick \& McKinney (2010). We do not detect any polarized jet emission in this source at our epoch (see online figure set of Figure 1, Figure 1.79) and therefore do not search for gradients. We do not obtain good $\lambda^{2}$-fits in the core but the few pixels where we obtain acceptable fits agree with the RM values of O'Sullivan \& Gabuzda (2009).

Contopoulos et al. (2009) list 29 sources for which a gradient can be seen in RM maps collected from the literature. Our sample includes 25 of these and we have looked for gradients in the 18 sources in which Contopoulos et al. (2009) report a gradient at least 2 mas from the core. These include 3C 273, $3 \mathrm{C} 454.3$, and $2230+114$ and two others that are in our list of sources showing hints of gradients. In the remaining 14 we either do not detect RM outside a beam width from the core (five sources) or do not see any indication of a gradient in 

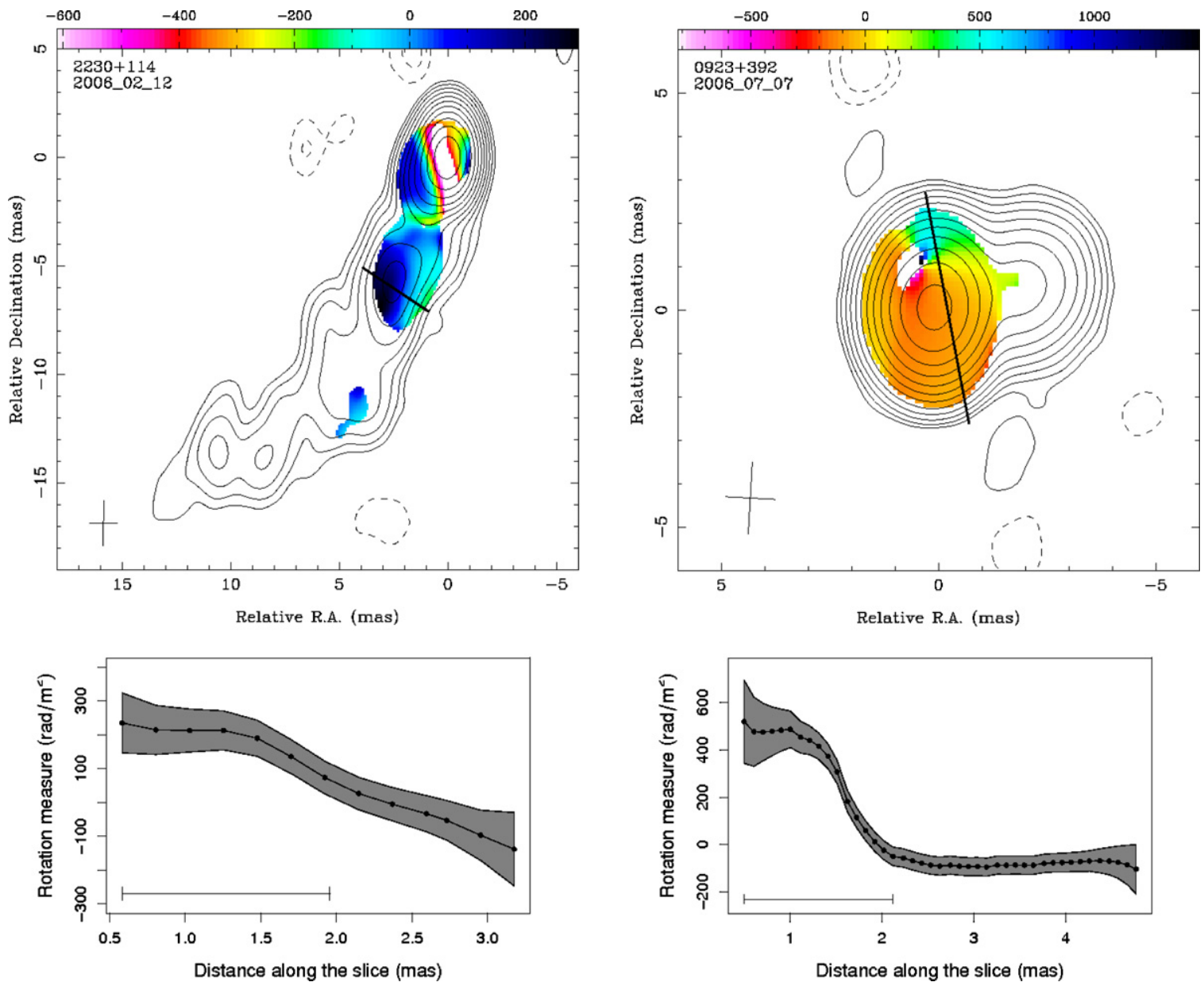

Figure 11. RM maps and transverse RM slices of 2230+114 (left) and 0923+392 (right). Beam size along the slice is shown in each plot with a scale bar.

(A color version of this figure is available in the online journal.)

the jet at our epochs. We note that some of these observations were done at longer wavelengths and therefore may have been more sensitive to polarization and Faraday rotation.

Based on our results of a very large sample of RM maps we conclude that robust mas-scale RM gradients are difficult to detect at $>3 \sigma$ level in the inner jets of AGNs. The major cause for this is that the majority of the jets we observe are not transversely well-resolved and therefore we do not consider the gradients as robust based on our detailed simulations in Appendix C.2. We note that multi-epoch observations may help to confirm other gradients because in principle, detections of three $2 \sigma$ gradients in the same source at the same location would give an overall significance of $3 \sigma$. However, this requires that the significance of the individual detections is determined using statistically correct error bars derived from error propagation of $Q$ and $U \mathrm{rms}$ error and accounting for additional D-term and CLEAN errors as described in Appendix B. In addition to the four sources where we detect significant transverse gradients, our sample includes only five other sources which have polarized jets wider than two beam widths and show no sign of a transverse gradient. The reduced sensitivity and angular resolution makes the detection of gradients very difficult in objects at higher redshifts. For example, if we restore the maps of 3C 273 with a beam size corresponding to angular resolution of a $z=0.5$ object and reduce the flux density by a factor of 10 to achieve a typical flux density of a source at $z=0.5$, the gradient would no longer be significant. Higher sensitivity and better angular resolution observations would help to solve the problem. Given the existing constraints of the current VLBI arrays, this is a strong science motivation for high-sensitivity space VLBI.

\subsection{Time Variability}

As was noted in the previous section, there is clearly variability in the RM values over timescales of years or even months. If the Faraday rotation is caused by an external screen very far away from the jet, for example, in intergalactic clouds or even in the narrow-line region of the AGN, the RMs observed in fixed locations (with respect to the core) in the jets of AGNs should remain constant over timescales of years. In order for external clouds to cause variations over timescales of years, they should be so small in size that the number density is not large enough to cause significant variations in the observed RM. Therefore, it is important to verify that the variations are seen in the same parts of the jet and are not due to changes in the jet position angle (Gómez et al. 2011).

Twenty sources in our sample were observed twice during the 12 month period, and in 10 AGNs we measured RM values in both of the epochs. Additionally, we can compare our observations to the 8-15 GHz observations of Taylor (1998, 2000) and Zavala \& Taylor $(2002,2003$, 2004) where RM maps for 36 sources in our sample, mainly quasars, are shown (3C 279 is shown in three of them). These observations were obtained between 1997 and 2000, and our comparisons are therefore affected by the long gap between our observations. 
Another factor that will affect at least some sources is that Zavala \& Taylor did not remove the contribution of Galactic Faraday rotation from their maps. This affects, for example, $2200+420$ where Zavala \& Taylor (2003) observe a jet RM of $-287 \mathrm{rad} \mathrm{m}^{-2}$, which will reduce to $-136 \mathrm{rad} \mathrm{m}^{-2}$ if the Galactic Faraday rotation is accounted for. This value is consistent within our error bars with our jet RM values of about $0 \mathrm{rad} \mathrm{m}^{-2}$. RM maps of five of our sources were obtained in 2006 July by O'Sullivan \& Gabuzda (2009), which allows us to probe shorter timescale variations. They include RM maps between 7.9 and $15 \mathrm{GHz}$ for most of their sources, close to our resolution. Out of these five, $0954+658$ and $1156+295$ were already discussed in the previous section.

\subsubsection{Variability in the Core RMs}

In four sources that were observed twice during our program $(0215+015,0716+714,0834-210$, and 0847-120), we detect $\mathrm{RM}$ values only in small regions or near the core and the values are all within $1 \sigma$ error bars in the two epochs. In $0219+428$ and $2200+420$ our observations agree very well. We do not detect good $\lambda^{2}$-fits in the cores of these two sources in either of the epochs. In the case of $2200+420$ this agrees well with observations of Mutel et al. (2005) who observed 2200+420 over nine epochs between 1997 and 2002 at 15, 22, and $43 \mathrm{GHz}$, and did not detect good $\lambda^{2}$-fits in four of their epochs. They showed that this is due to blending of multiple components in the core region. The components were seen as separated in their 22 and $43 \mathrm{GHz}$ observations but were blending together when restored with the $15 \mathrm{GHz}$ beam. This could also be the case in $1418+546$, where four Gaussian components are required to fit the region 2 mas from the core at $15 \mathrm{GHz}$. O'Sullivan \& Gabuzda (2009) also observed this source in 2006 July between 4.6 and $43 \mathrm{GHz}$. In our 2006 February epoch we obtain a similar core RM value as they do, although we do not detect the positive RM patch as seen in their 7.9-15.3 GHz map. In our 2006 November map the core values change by $200 \mathrm{rad} \mathrm{m}^{-2}$, compared to our previous epoch and their map from July. In this epoch, we also detect the positive RM patch seen in their map.

O’Sullivan \& Gabuzda (2009) observed 1749+096 in 2006 July, but they do not obtain any good $\lambda^{2}$-fits, which is surprising because we detect RM values in the core of $1749+096$ in our June epoch, observed only two weeks earlier. They attribute the inability to obtain good fits to a possible flare in the source that may affect the polarization structure. Indeed, their peak flux in total intensity in the $7.9 \mathrm{GHz}$ band is $4.2 \mathrm{Jy} \mathrm{beam}^{-1}$ while in our $8.1 \mathrm{GHz}$ observation it is $3.5 \mathrm{Jy} \mathrm{beam}^{-1}$ so that a flare is probably ongoing and could affect their July observations. In fact, a flare peaking in 2006 October is seen in the $15 \mathrm{GHz}$ MOJAVE observations. ${ }^{15}$ Additionally, our wavelength coverage is not as wide as theirs so it is possible that we do not detect the non- $\lambda^{2}$ behavior they see.

Variations in the core RMs are also seen in comparison of our observations to those of Taylor $(1998,2000)$ and Zavala \& Taylor (2003, 2004). In general, the core values between our maps and theirs differ significantly and there are only five sources where we detect similar core RM values. This can also be attributed to the blending of components within the finite beam. Alternatively, if the Faraday rotation is internal to the jet, changes in the particle density or magnetic field strength due to newly emerging components could cause the variations. In

\footnotetext{
15 http://www.physics.purdue.edu/astro/MOJAVE/sourcepages/ 1749+096.shtml
}

six sources the core RM in our maps has a different sign than in their observations which indicates a change in the direction of the line-of-sight component of the magnetic field. However, due to the long gap between our observations and the complexity of the core regions in these sources, it is difficult to distinguish if this is due to a sign reversal in an ordered magnetic field or due to changes in properties of new components.

\subsubsection{Variability in the Jet RMs}

3C 273 and 3C 454.3 were seen to have large variations in the jet RMs over timescales of three month in our observations, which was discussed in the previous section. In some sources it is clear that we are seeing a different part of the jet at successive epochs and sometimes we do not detect the polarized jet emission at all, or vice versa. This is seen, for example, in complex sources such as 1253-055 and 1418+456 where we do not see the exact same part of the jet because the same jet components are not detected or the components have moved significantly between the two epochs.

In many sources we still see fairly similar jet RM values within the error bars. In the jet of $2200+420$ we observe RM values consistent with $0 \mathrm{rad} \mathrm{m}^{-2}$, in agreement with Mutel et al. (2005). This source was observed by O'Sullivan \& Gabuzda (2009) in 2006 July between 4.6 and $43 \mathrm{GHz}$ where they obtain slightly higher RM values in the jet than we do. However, these are still for the most part within the error bars of our observations. Only in 2230+114 do we see differing values in the jet RM, even though it looks like we are looking at the same portions of the jet. Taylor (2000) detects an RM of $-185 \mathrm{rad} \mathrm{m}^{-2}$ in the jet of $2230+114$ (component $\mathrm{C}$ in that paper), which is almost at the same location as our component 3 with RM of $+173 \mathrm{rad} \mathrm{m}^{-2}$. The different sign in the observed RMs indicates a change of direction in the line-of-sight component of the magnetic field. It will be interesting to compare these to our follow-up observations to determine if the sign has remained the same.

Even though at first glance it looks like the RM values in the jets of AGNs change over timescales of several years, a detailed comparison taking into account the differing locations of the polarized components shows that we do not detect significant variations in the jet RMs of the majority of AGNs. The fact that we detect non-zero RMs in the jets even after the Galactic Faraday rotation contribution is taken into account suggests that the RM is occurring outside our own Galaxy, for example, in the narrow-line region of the AGN (e.g., Zavala \& Taylor 2003). In three sources that show signs of transverse rotation measure gradients, 3C 273, 2230+114, and 3C 454.3, RM time variations are seen in the jet as well. In 3C 273 and 3C 454.3 the variations happen over timescales of three months, pointing to either internal Faraday rotation or interaction between the jet and the Faraday rotating material, possibly a sheath around the jet, as discussed in more detail in Section 4.3. If the rotation is internal, the rotating plasma is either the low-energy end of the relativistic electron population or thermal plasma intermixed with the emitting plasma, and therefore fast changes in the magnetic field of the jet can cause fast variations in the observed RM. If the jet is surrounded by a sheath, the sheath must be mildly relativistic because we do not detect counter-jet emission in these sources. Therefore, over timescales of years, it is possible that the RM variations are caused by changes in the screen itself (e.g., Asada et al. 2008a). This could explain the variability in $2230+114$, but not in the two other sources where the gas in the sheath would not have 

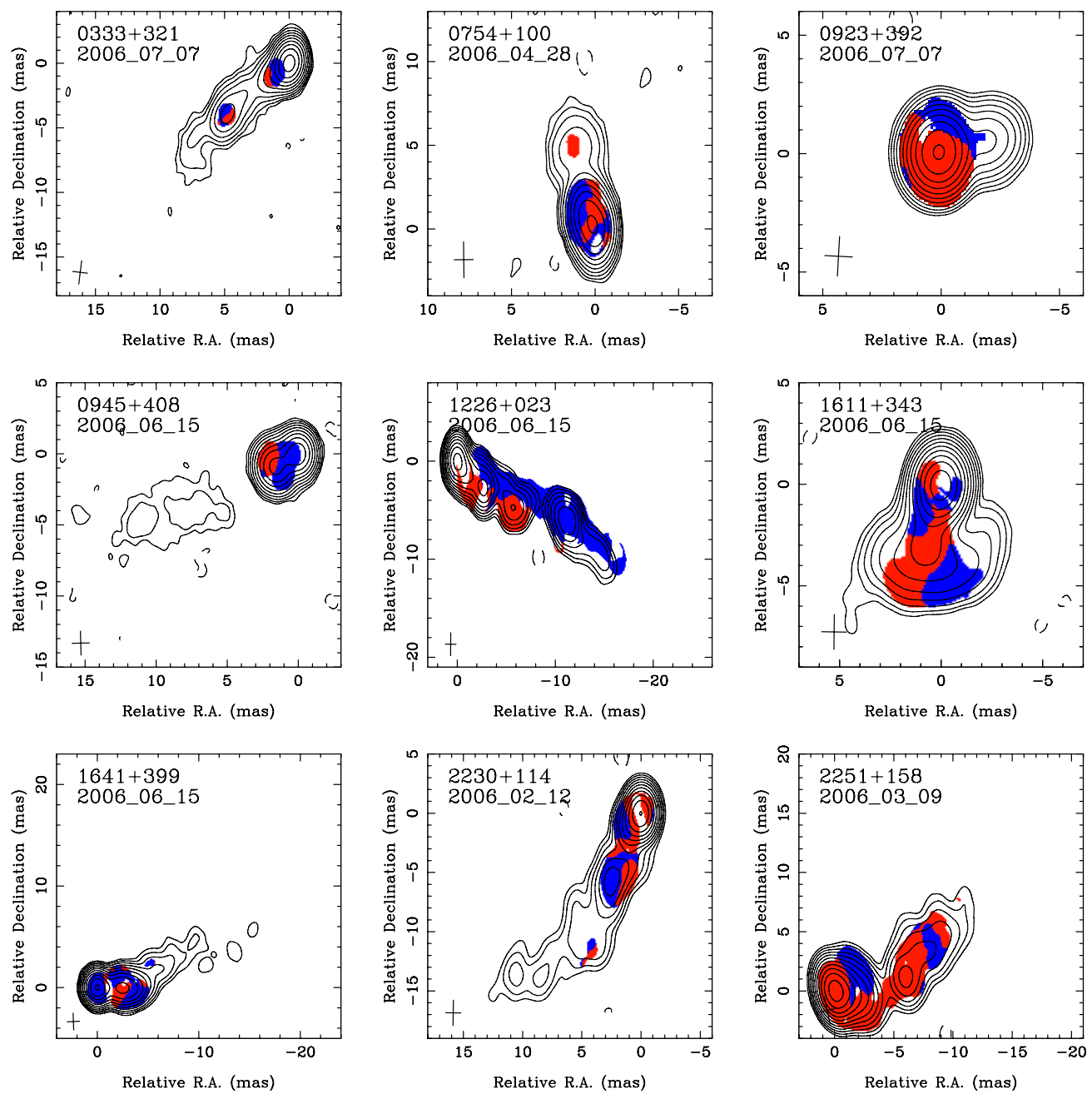

Figure 12. Maps of the rotation measure sign in nine example objects showing bilateral structure (see the text for details). Dark gray (blue) areas are positive RM and light gray (red) areas negative RM.

(A color version of this figure is available in the online journal.)

moved sufficiently over the timescale of three months to cause significant variations. In sources with fast apparent superluminal motion, it may be possible to observe variations over timescales of months when the components illuminate a different part of the Faraday screen (e.g., Gómez et al. 2011). In these sources the same RM should be seen when another component passes the exactly same part of the jet, which could then be studied with frequent multi-epoch observations. This seems not to be the case in 3C 273 or 3C 454.3 where the components have only moved about 0.1 mas between the two epochs. We note that if the variations happen only in small parts of the jets, our ability to detect variability is easier for nearby sources with resolved jets.

\subsection{Comparison to Simulations}

Recently, using GRMHD simulations, Broderick \& McKinney (2010) produced simulated RM maps for AGN jets with large-scale toroidally dominated magnetic fields. They produced maps with different resolutions and beam sizes to additionally study the effect of finite beam size on RM observations. Their results showed that any RM gradients observed within a beam width from the core are unreliable at resolutions below $43 \mathrm{GHz}$ at VLBA. Further down the jet it should be possible to detect true RM gradients if the magnetic field is toroidal. Formally, with infinite sensitivity, even in unresolved jets it may be possible to detect gradients of the right sign, with the magnitude severely suppressed. They did not, however, take into account the effect of noise in their simulations, which we have shown in Appendix C.2 to have a major effect on the reliability of gradients in unresolved jets. They also showed that if the toroidal component of the field is made to vanish, any significant gradients will vanish too. They conclude that linear, resolved transverse gradients are due to large-scale toroidally dominated magnetic fields within the Faraday screen. This provides us a way to compare our observations with their simulations. It is difficult to make quantitative comparisons, but Broderick \& McKinney (2010) show that even maps of the RM sign should show the bilateral structure if the toroidal magnetic field component dominates. Their Figure 12 shows examples of sign maps for different resolutions and other parameters, such as the jet viewing angle and black hole mass. Additionally, they present a model in which the toroidal field is made to vanish, and a random foreground Faraday screen is inserted.

In nine sources visual inspection of the sign maps reveals clear bilateral structures as shown in Figure 12. These include 3C 273, $3 \mathrm{C} 454.3,0923+392$, and $2230+114$ in which the gradient is significant based on our detailed analysis in Section 4.3. In two other sources $(0945+408$ and $1641+399)$, in we which detect 

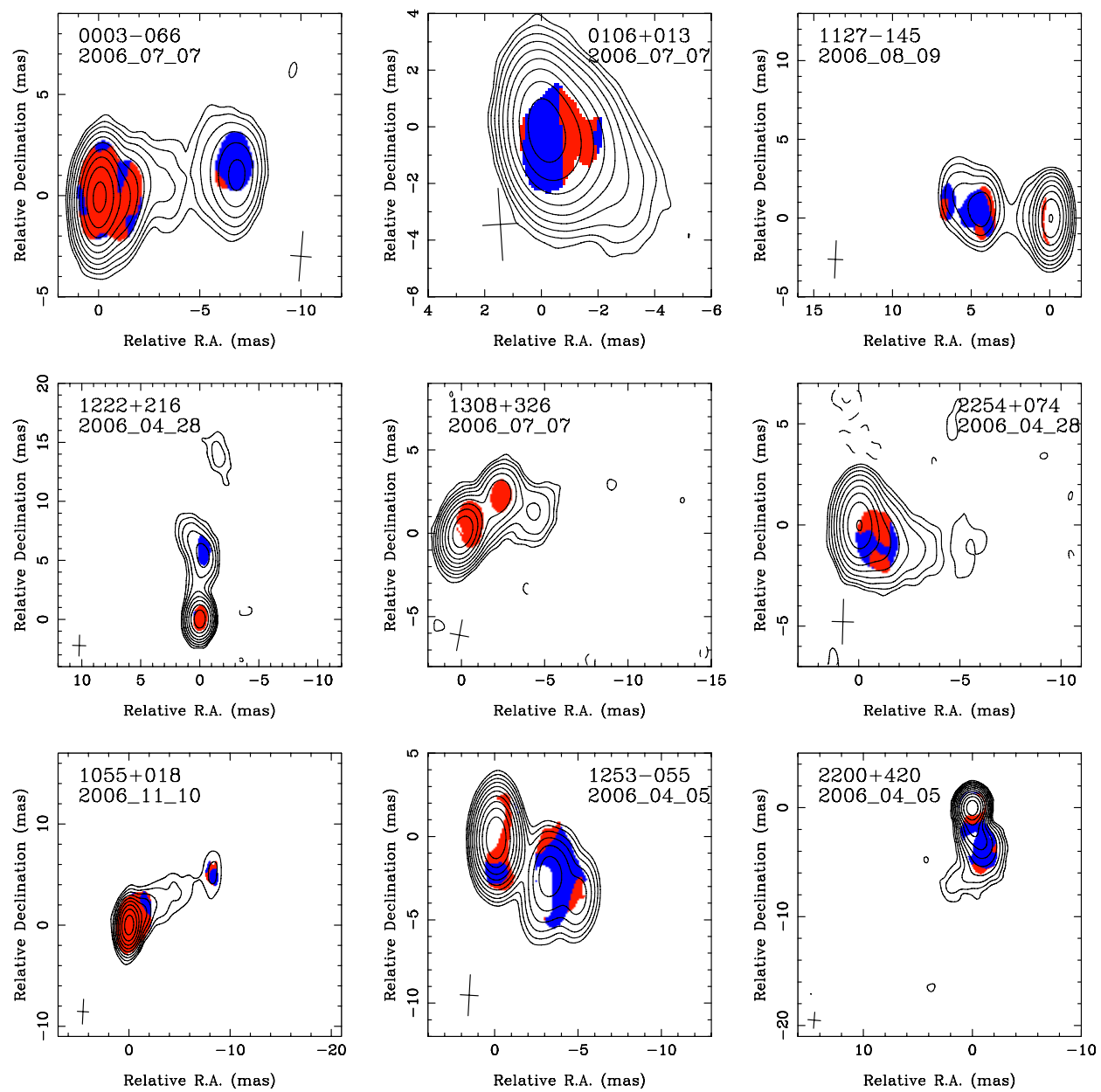

Figure 13. Maps of the rotation measure sign in nine example objects that represent the typical sources in our sample (see the text for details). Dark gray (blue) areas are positive RM and light gray (red) areas negative RM.

(A color version of this figure is available in the online journal.)

the gradient at a $2 \sigma$ level, the sign maps show different signs on both sides of the jet along the whole polarized region of the jet. These are candidate sources for toroidally dominated large-scale magnetic fields. However, one should note that for example in the case of $1611+343$, even though the sign map shows a bilateral structure, any possible gradient is within the $1 \sigma$ error bars, and the jet is over four beams wide at its widest location. In $0333+321$ and $0754+100$ the jets are not sufficiently resolved for us to make any conclusions about the significance of the gradient. If the jets are not viewed directly from the side in the jet frame, it may be that we are seeing only the positive or negative side of the possible toroidal field. Therefore, we have produced sign maps where the median jet region RM is subtracted from the map to see if we can detect more sources with bilateral structures. There are only two such sources, $1150+812$ and 2005+403. Reichstein \& Gabuzda (2012) report a sign change centered around zero in $1150+812$ in lower frequency data between 4.6 and $8.9 \mathrm{GHz}$, while in our map the center is about $240 \mathrm{rad} \mathrm{m}^{-2}$. In our map the change in magnitude also happens nearly transverse to the jet while in their map it is more along the jet. The jet of $1150+812$ is only one beam width wide in our observations and therefore we cannot make conclusions about the significance of the gradient. In $2005+403$ the jet is 1.5 beams wide in polarization and the values are centered around $\sim 110 \mathrm{rad} \mathrm{m}^{-2}$, but are all within one $\sigma$ of each other and therefore the gradient is not significant.
In Figure 13 we show nine example cases that we believe represent typical types of behavior in the sign maps. In many sources we see how the RM sign changes along the jet direction (e.g., 0003-066 and 1222+216). These kind of structures look similar to simulations of unresolved jets with zero toroidal magnetic field component or random magnetic field. Multiepoch studies of changes in the RM and polarization structure could give more insights into the nature of the screen and if it is internal or external to the jet. In the bottom row we show examples of sources in which the jet is transversely resolved but the sign maps show a very complex structure similar to the simulated case with a resolved jet and zero toroidal magnetic field or random foreground screens (e.g., 1253-055) or no change in the sign of the RM. The majority of the sources in our sample show sign maps similar to Figure 13 or sign maps without any change in the sign. We note, however, that the majority of our jets are transversely unresolved and therefore it may be possible that we simply cannot detect the bilateral structure in our maps.

\section{CONCLUSIONS}

We have observed a sample of 191 AGN jets (20 at two epochs) at 8.1, 8.4, 12.1, and $15 \mathrm{GHz}$ with VLBA to determine the Faraday rotation measures in the parsec-scale jets of these sources. One motivation for the study was to find out how much Faraday rotation would affect the EVPAs of the MOJAVE 
program observations at $15 \mathrm{GHz}$. The polarization is sufficiently strong to study Faraday rotation in 149 sources (159 maps), and in over $80 \%$ of the sources the median observed RM is less than $400 \mathrm{rad} \mathrm{m}^{-2}$, which would rotate the $15 \mathrm{GHz}$ EVPAs by less than $10^{\circ}$. Additionally, we do not see significant variations in the jet RMs of most of the sources so that these results can be extrapolated to other MOJAVE epochs as well. However, there are several sources with high observed RMs of larger than $1000 \mathrm{rad} \mathrm{m}^{-2}$ so that when studying polarization in individual sources (especially at frequencies lower than $15 \mathrm{GHz}$ ), one needs to take the Faraday rotation into account. The highest intrinsic redshift-corrected absolute RM $8975 \mathrm{rad} \mathrm{m}^{-2}$ is detected in a jet component 0.5 mas from the core in the high-redshift quasar $0642+449$ at $z=3.396$.

We find the quasars to have generally higher absolute RMs than the BL Lac objects (median $\left|\mathrm{RM}_{\mathrm{obs}}\right| 144 \mathrm{rad} \mathrm{m}^{-2}$ versus $79 \mathrm{rad} \mathrm{m}^{-2}$ ) and the core components in the sources to have higher RMs (median $\left|\mathrm{RM}_{\mathrm{obs}}\right| 171 \mathrm{rad} \mathrm{m}^{-2}$ ) compared to the jets (median $\left|\mathrm{RM}_{\mathrm{obs}}\right| 125 \mathrm{rad} \mathrm{m}^{-2}$ ), which is also seen in both quasars and BL Lac objects separately. In quasars, we also detect a significant negative correlation with the magnitude of the RM and the de-projected distance of the component from the core.

We perform detailed simulations of the measurement error of the RM, and focus especially on the effect of noise and finite restoring beam size on putative transverse RM gradients. Because of the finite beam size, neighboring pixels are not independent and this has to be taken into account when interpreting radio interferometric images. For a typical jet there are only few independent measurements across a jet of apparently many pixels. Our simulations show that it is possible to obtain spurious yet significant-looking transverse RM gradients if the polarized region in the jet is less than two beams wide. We give several guidelines on how the errors in RM maps need to be taken into account to determine the reliability of a transverse gradient. The reliability is strongly dependent on the jet width, and the jet preferably should be two beams wide, and the gradient more than $3 \sigma$ in significance, to call reliable. The errors in the RM maps should be calculated from the variance-covariance matrix of the least-squares fit where the EVPA errors are determined using error propagation from Stokes $Q$ and $U$ rms errors, additionally accounting for effects due to the CLEAN procedure. Following these guidelines, we detect significant transverse RM gradients in the flat spectrum radio quasars 3C 273, 3C 454.3, $0923+392$, and $2230+114$. In 3C 273 the RM is for the first time seen to change sign along the transverse slice, giving further support for a helical magnetic field in the jet. The main reason why we are not able detect gradients in more sources is that the jets are insufficiently resolved at our lowest observing frequency of $8.1 \mathrm{GHz}$. In addition to these four sources, there are only five which have wide-enough polarized jets where a gradient is not detected. The reduced sensitivity and angular resolution makes the detection of gradients very difficult in objects at higher redshifts. Higher angular resolution observations of much greater sensitivity are needed to study this phenomenon further.

Comparison of our RM maps to earlier studies confirm that significant temporal RM variations in the core components of AGNs are common. This could be due to multiple polarized components blending within the finite beam at different times or intrinsic changes in the magnetic field or particle density if the rotation is internal to the jet. Higher resolution observations at higher frequencies are required to uncover the true RMs in the cores of many of the sources. In almost all the cases where our jet RM values differ from earlier studies, it can be explained with different part of the jet being illuminated by the components at different times. Only in 3C 273, 3C 454.3, and $2230+114$ do we detect variations which cannot be explained by moving components. In $2230+114$ the variations happen on timescales of years, which could be possible if the Faraday rotation occurs in a mildly relativistic sheath around the jet. In 3C 273 and 3C 454.3 we see variations over a timescale of three months that are difficult to explain with external Faraday rotation models, so more likely the rotation is internal to the jet. This is further supported by observations of inverse depolarization structures in the jet components of these two sources, which show the $8 \mathrm{GHz}$ polarization to be higher than at $15 \mathrm{GHz}$. This is the opposite trend with wavelength expected from standard external depolarization and may be explained by combining internal Faraday rotation with helical or loosely tangled random magnetic fields (Homan 2012). However, in the majority of isolated jet components we studied, the relationship between depolarization and RM can be explained with a purely random external Faraday screen viewed through a small number of lines of sight. In these sources the rotation could be caused by intergalactic clouds or the narrow-line region of the AGN.

We thank the anonymous referee for the many suggestions that helped to improve the paper. The authors acknowledge the other members of the MOJAVE team. We thank D. Gabuzda, G. Taylor, and K. Wiik for useful discussions. The MOJAVE project is supported under National Science Foundation grant AST- 0807860 and NASA Fermi grant NNX08AV67G. Work at UMRAO has been supported by a series of grants from the NSF and NASA and by funds for operation from the University of Michigan. T. Hovatta was supported in part by the Jenny and Antti Wihuri foundation. D. Homan was funded by National Science Foundation grant AST-0707693. Part of this work was done when T. Savolainen and Y. Y. Kovalev were research fellows of the Alexander von Humboldt Foundation. Y. Y. Kovalev was supported in part by the Russian Foundation for Basic Research (grant 11-02-00368) and the Dynasty Foundation. The National Radio Astronomy Observatory is a facility of the National Science Foundation operated by under cooperative agreement by Associated Universities, Inc. This research has made use of NASA's Astrophysics Data System, and the NASA/IPAC Extragalactic Database (NED). The latter is operated by the Jet Propulsion Laboratory, California Institute of Technology, under contract with the National Aeronautics and Space Administration.

Facilities: VLBA, UMRAO, VLA

\section{APPENDIX A}

\section{EFFECT OF IMAGE ALIGNMENT ON RM MAPS}

To study the effect of image alignment errors on the rotation measure maps, we used 12 sources for which we have a very good correspondence between the shifts from our 2D crosscorrelation and from optically thin component positions. In all these sources the difference between the two shifts is less than 0.1 mas. We assumed the shifts in these cases to be correct and introduced artificial shifts between the maps to study the effect on both spectral index and rotation measure maps. The shifts were applied to the maps of all the other frequency bands with respect to the $15.3 \mathrm{GHz}$ maps. In the case of spectral index maps, the effect was large, with some sources showing clear differences with shifts as small as 0.03 mas. In other sources, even a shift of 0.18 mas, almost twice our median shift for the 


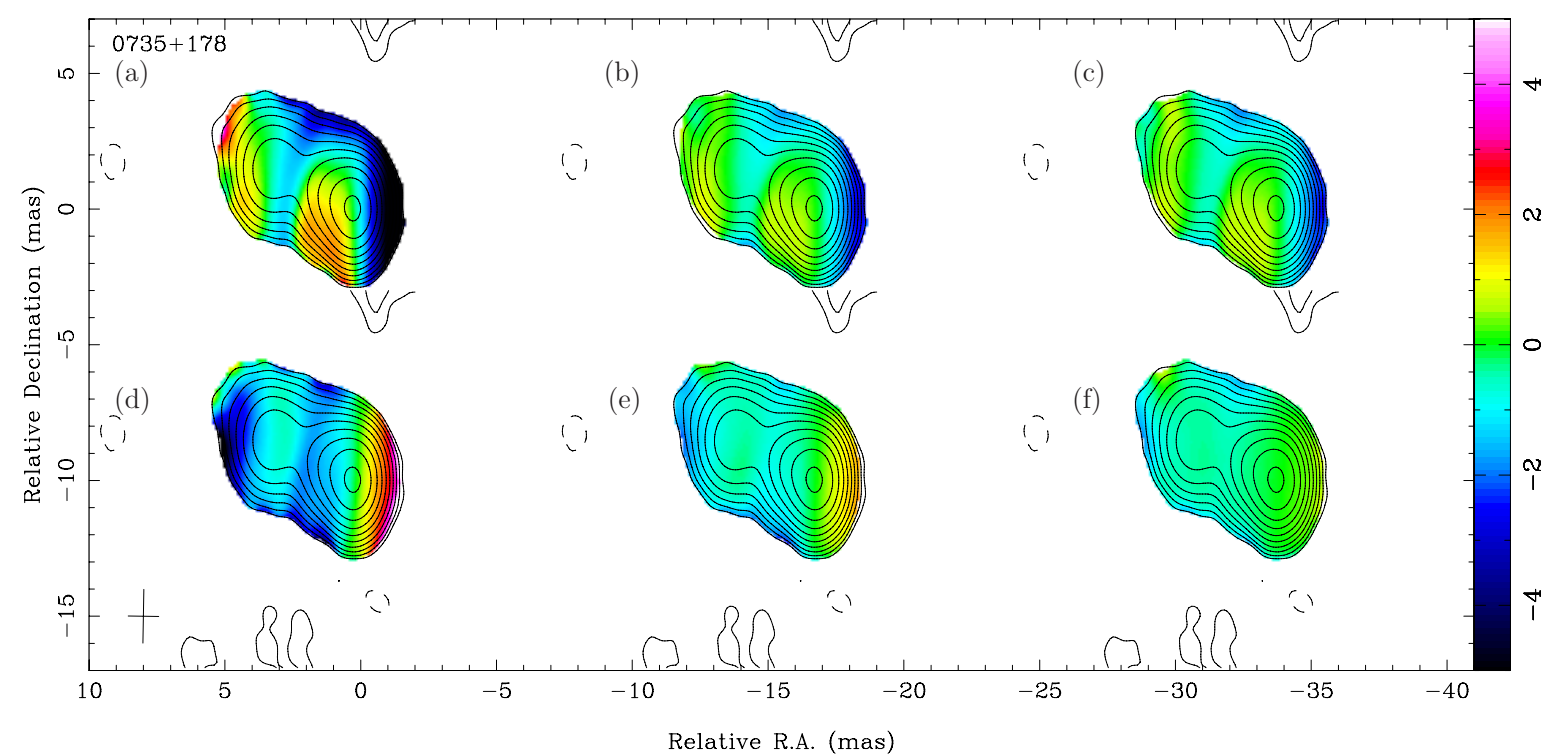

Figure 14. Spectral index maps between 15 and $12 \mathrm{GHz}$ ((a) and (d)), 15 and $8.4 \mathrm{GHz}((\mathrm{b})$ and (e)), and 15 and $8.1 \mathrm{GHz}((\mathrm{c})$ and (f)) aligned by the map's center (top panels (a)-(c)), and by using correct shifts from the 2D cross-correlation (bottom panels (d)-(f)). Contours correspond to the total intensity at $15 \mathrm{GHz}$.

(A color version of this figure is available in the online journal.)

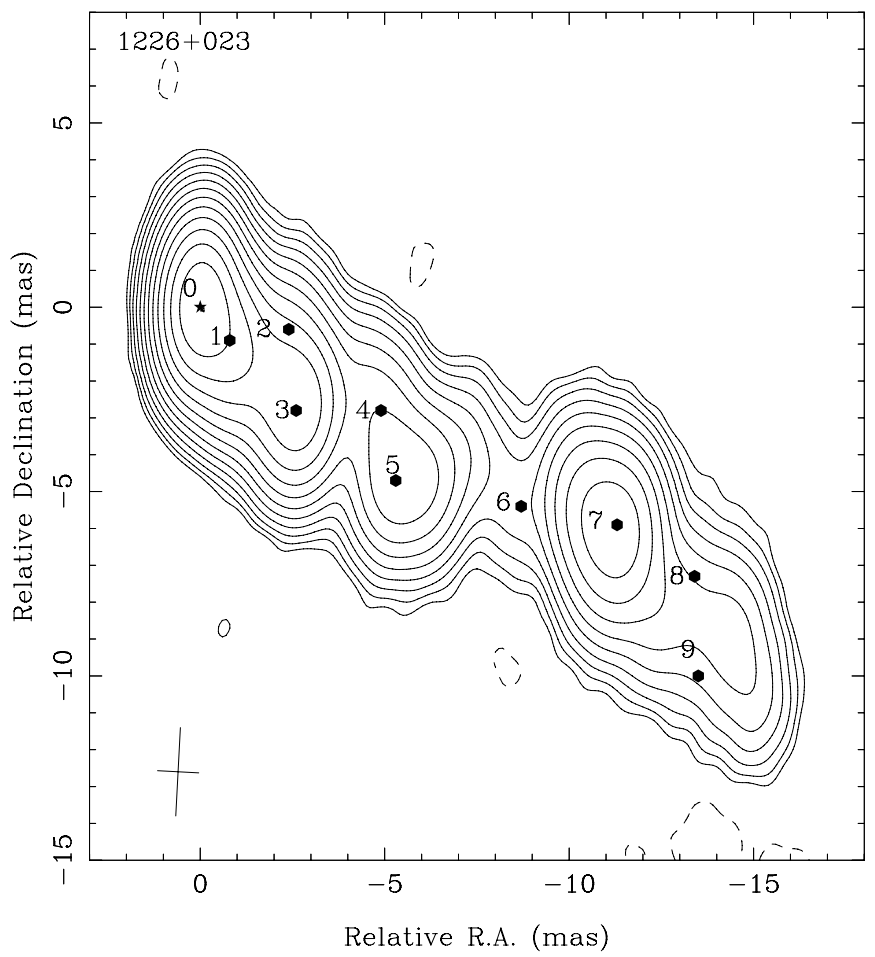

Figure 15. Locations of the simulated distributions in the jet of $1226+023$ overlaid on the $15 \mathrm{GHz}$ total intensity contours. Star shows the component discussed in the error analysis.

whole sample, did not cause major changes in the spectral index maps. For each of the test sources we calculated rotation measure maps for shifts that were seen to create spurious features in the spectral index maps. The effect on the rotation measure maps was much smaller, and shifts as large as 0.18 mas did not affect the general structure. In most of the cases small differences could be seen in the edge pixels or close to patches of low signal to noise, which in any case are considered unreliable. In only two maps did we see appreciable differences in the jet rotation measure, and in these cases the wrong shifts were 0.09 and 0.15 mas, but those were also in very complicated

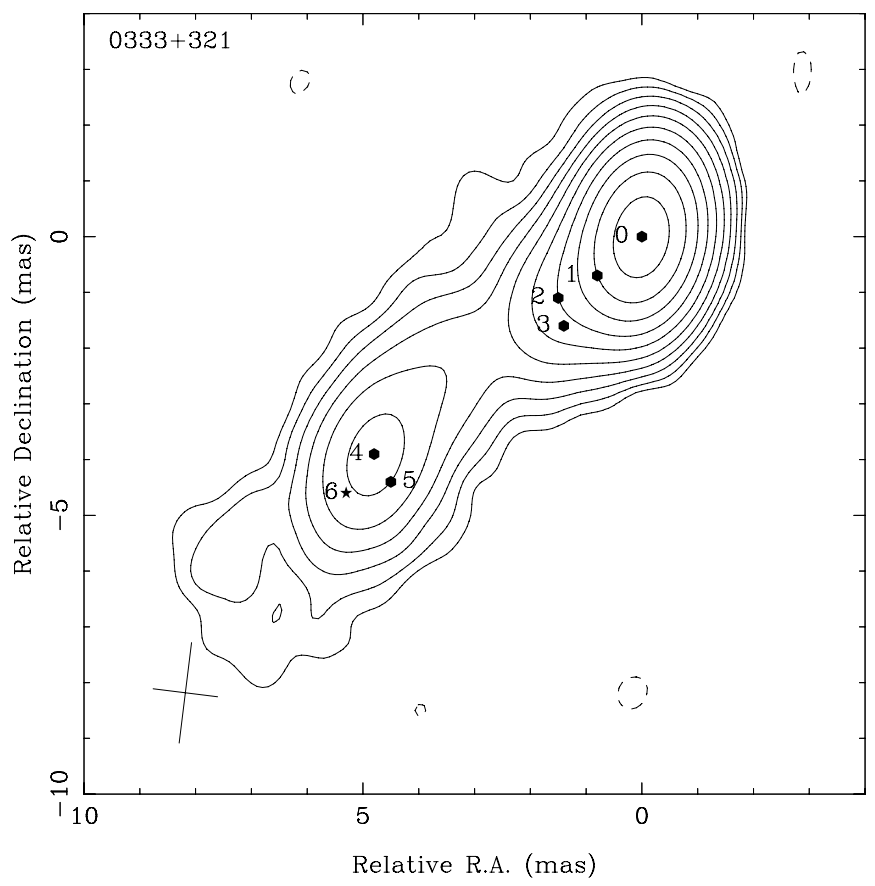

Figure 16. Locations of the simulated distributions in the jet of $0333+321$ overlaid on the $15 \mathrm{GHz}$ total intensity contours. Star shows the component discussed in the error analysis.

regions of the jet. Even in these two cases the general structure of the RM map did not change and our conclusions would be unaffected. Therefore, we conclude that even if our image alignment is off by 0.15 mas it should not affect the results from our rotation measure maps, especially as we are not using the edge or low signal-to-noise regions to make conclusions about the RM structure. By using the spectral index map as an additional indicator of the goodness of the alignment we ensure that our rotation measure maps are not affected by false alignment between the different bands.

An example of spectral index maps in the case where the alignment is very important is shown in Figure 14. The figure shows spectral index maps without and with shifting between 


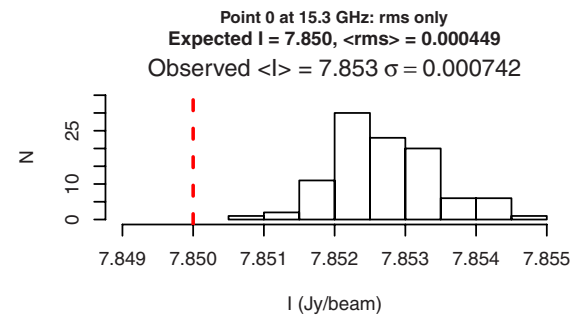

I (Jy/beam)

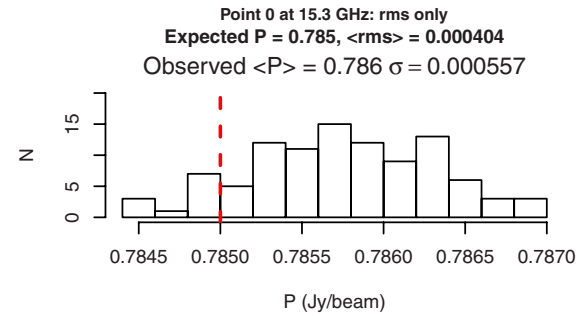

$\mathrm{P}$ (Jy/beam)
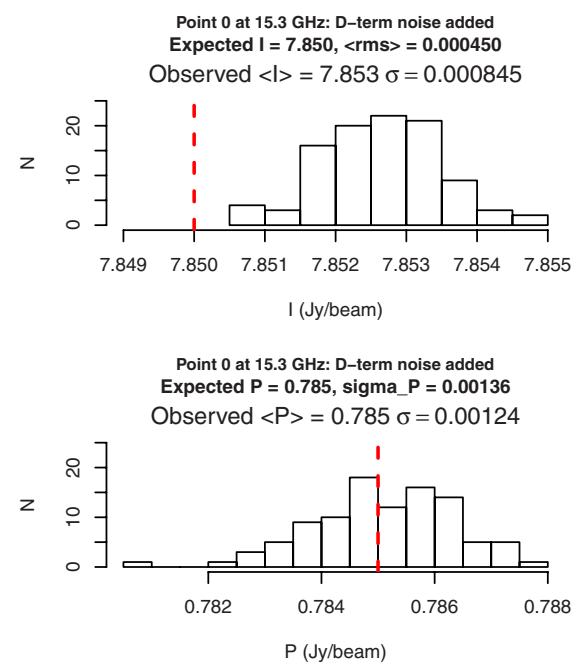

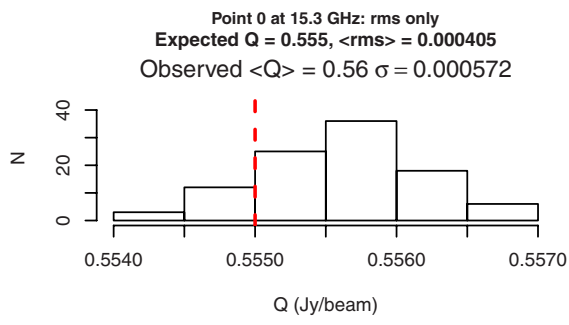
Expected $Q=0.555,<$ rms $>=0.000405$ Observed $\langle Q\rangle=0.56 \sigma=0.000572$
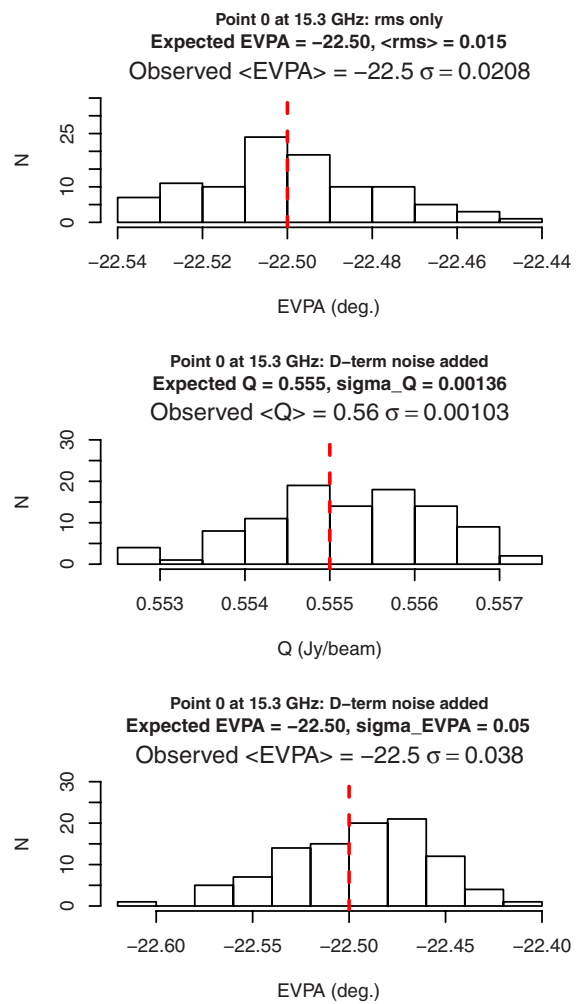
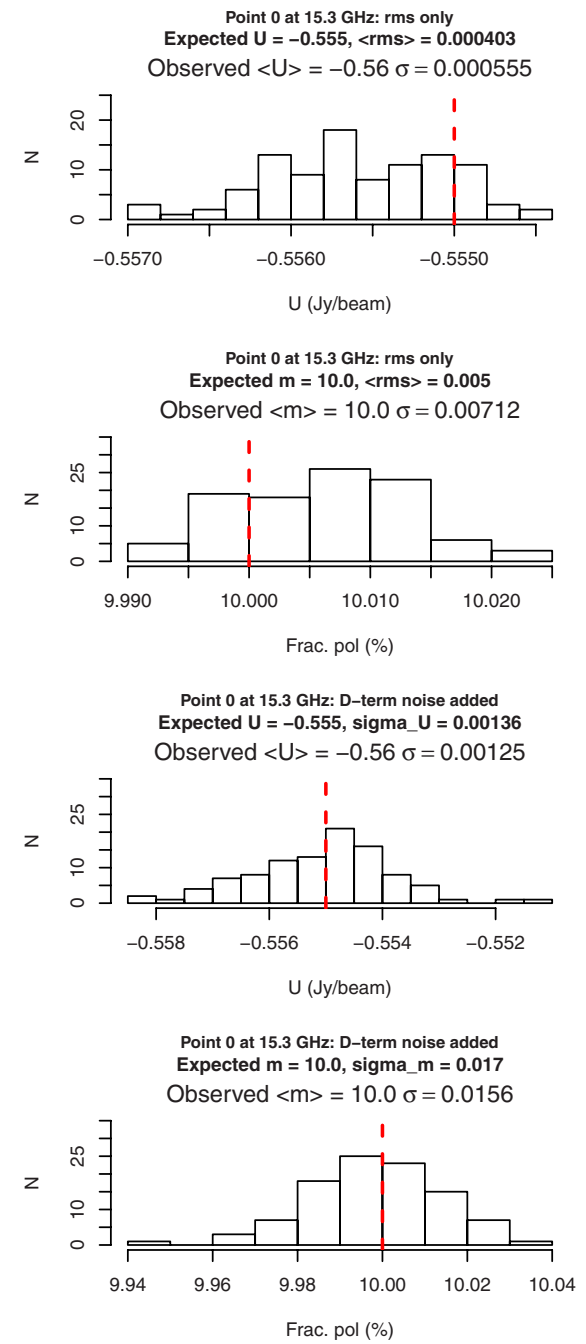

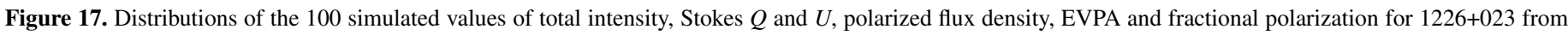

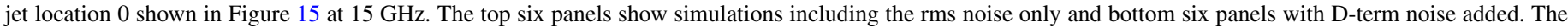
expected value is shown by a dashed line (red in the online journal). See the text for information on the expected error values.

(A color version of this figure is available in the online journal.)

all the bands. The correct shifts were obtained using the 2D cross-correlation and are 0.39 and 0.03 mas in right ascension and declination between the 15 and $12 \mathrm{GHz}$ bands, 0.45 and 0.03 mas between the 15 and $8.4 \mathrm{GHz}$ bands and 0.33 and 0.03 mas between the 15 and $8.1 \mathrm{GHz}$ bands, and are shown in the bottom panel of Figure 14. Analogous to results of Kovalev et al. (2008), it is clear that the maps without shifting that have been aligned based on the phase center (top panel of Figure 14) have spurious features, such as optically thin regions apparently upstream of the core and a spectral index gradient transverse to the jet, which all disappear when the images are properly aligned.

\section{APPENDIX B}

\section{ERRORS IN POLARIZED FLUX DENSITY, FRACTIONAL POLARIZATION AND EVPA}

Errors in polarized flux density $\sigma_{p}$ are typically assumed to be the average rms error in the $Q$ and $U$ images. Additional sources of error are errors due to the CLEAN procedure which can be difficult to overcome (e.g., Lister et al. 2001) and errors due to instrumental polarization (D-terms) which are not evenly distributed across the images (Roberts et al. 1994). We used simulations to examine whether the error estimates we use are consistent with the above error contributions to the data. The simulations were carried out in several steps:

1. A Stokes $I$ model of the source was created using calibrated $(u, v)$ data of a real source and CLEAN in Difmap.

2. Stokes $Q$ and $U$ models were created by setting $Q$ and $U$ to be known fractions of Stokes I for each CLEAN component. Without any noise added, this corresponds to a uniform fractional polarization and EVPA across the source.

3. The original $(u, v)$ data were loaded into AIPS and the task UVMOD was used to replace the real data with the values produced in the previous step. Additionally, random noise of the same order as seen in our real data was added.

4. The UVMOD task was repeated 100 times to produce 100 simulated $(u, v)$ data sets with random noise added.

5. In the case of D-term simulations, additional random D-term error with a standard deviation of 0.002 , determined from the scatter in the D-terms of our data (see Section 2.2 for details on the determination of the stable D-terms), was added to each set using the task SPLIT. 


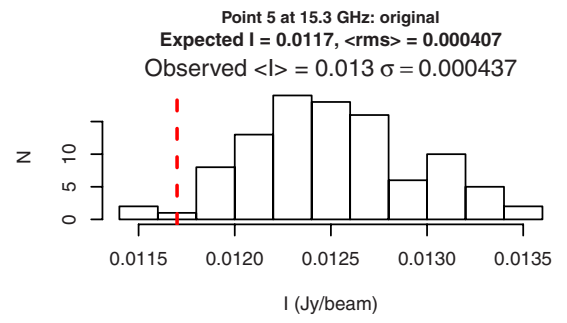

Point 5 at $15.3 \mathrm{GHz}$ : original Expected $\mathrm{P}=\mathbf{0 . 0 0 1 1 7},\langle\mathrm{rms}>\mathbf{=} \mathbf{0 . 0 0 0 4 2 5}$ Observed $\langle\mathrm{P}\rangle=0.0018 \sigma=0.000417$

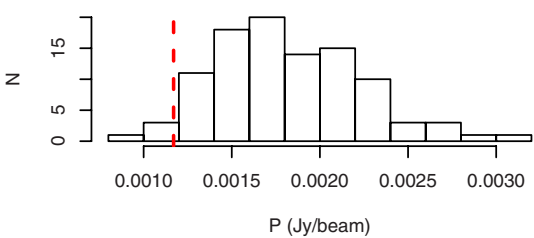

Point 5 at $15.3 \mathrm{GHz}$ : clipped and restored Expected $\mathrm{I}=\mathbf{0 . 0 4 1},<\mathrm{rms}>\mathbf{=} \mathbf{0 . 0 0 0 4 3 4}$ Observed $<\mathrm{l}>=0.04 \sigma=0.000519$

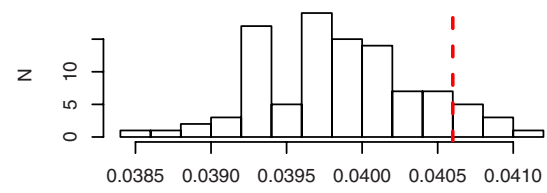

I (Jy/beam)

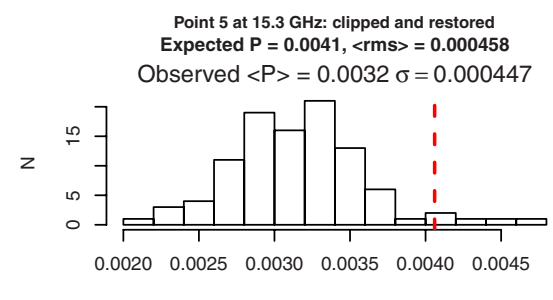

$\mathrm{P}$ (Jy/beam)

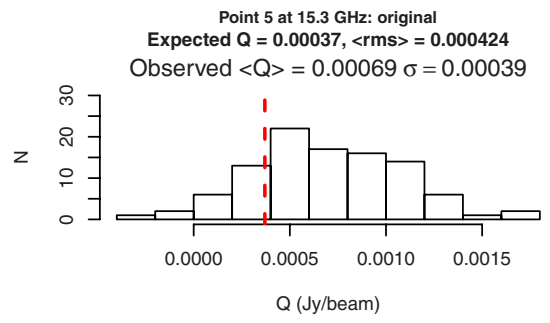

Point 5 at $15.3 \mathrm{GHz}$ original Expected EVPA $=35.71,\langle$ rms $>=10.4$

Observed $<$ EVPA $>=33.7 \sigma=5.99$
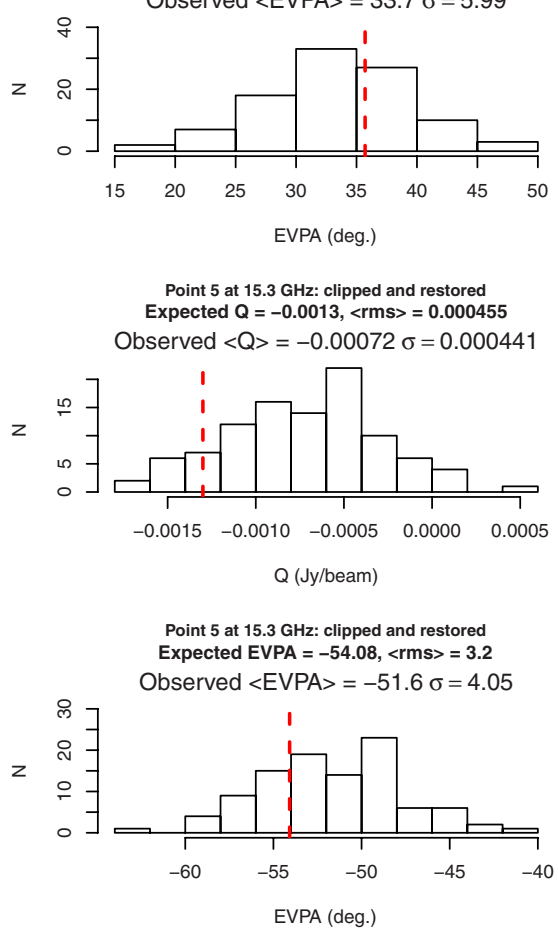
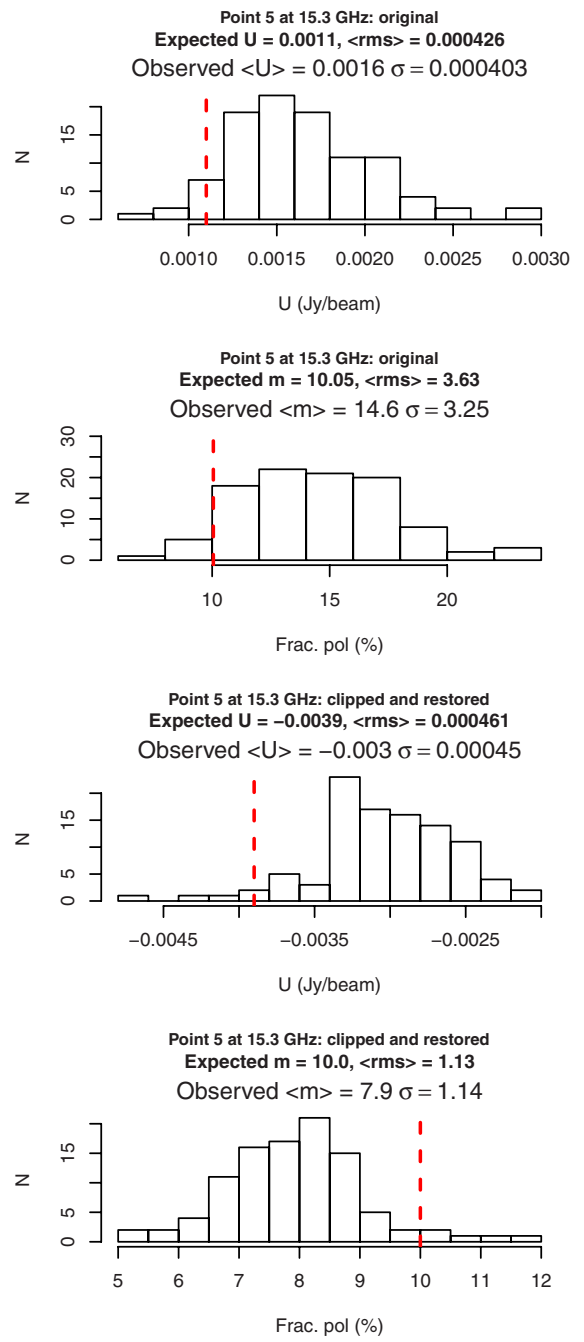

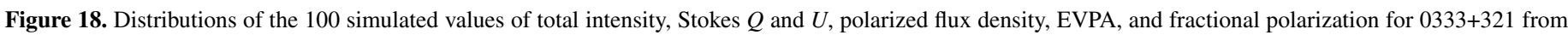

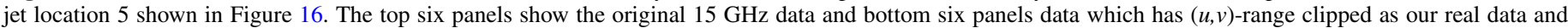

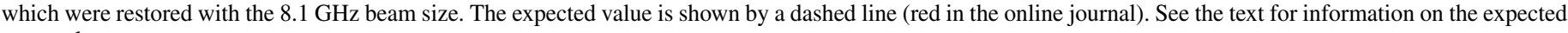
error values.

(A color version of this figure is available in the online journal.)

6. The simulated $(u, v)$ data were then imaged in Difmap following the same procedure as for the real data to obtain 100 images in Stokes $I, Q$, and $U$.

7. The rms in each image was obtained by shifting the map by 1 " and calculating the rms using the command "imstat" in Difmap.

The process was repeated for two sources, $0333+321$ and $1226+023$, to verify that the errors are general and do not depend on the specific structure of a given source. In order to address the errors due to the CLEAN procedure and the clipping of the $(u, v)$ coverage of our real data (see Section 2.1), the simulations were repeated for the original unclipped $15 \mathrm{GHz}$ data and the data at all the frequency bands were treated with the same cutoffs as our real images. We then studied the distributions of polarized flux density, fractional polarization and EVPA in individual jet locations shown in Figures 15 and 16. We chose locations at both the brightest parts of the jet and at the jet edges to see how well the error formula reproduces the standard deviation of the simulations.

For each location we determine the expected value and its expected error for all the parameters. For $I, Q$, and $U$ images the expected values are determined from low noise images, which have been created using the same simulation procedure but adding only $1 \%$ of the typical noise in the task UVMOD. Additionally, the last CLEAN step (6) was replaced by a simple restoration of the model components from step 1 to have comparison images which do not suffer from CLEAN errors. The expected rms value is taken from the simulated maps with noise added at a location 1 arcsec from the map center. The expected $p$, EVPA and $m$ values are calculated from the low noise images with $p=\sqrt{Q^{2}+U^{2}}, \mathrm{EVPA}=1 / 2 \tan ^{-1}(U / Q)$ and $m=p / I$. The expected error values are calculated using the following equations:

$$
\begin{gathered}
\sigma_{p}=\frac{\sigma_{Q}+\sigma_{U}}{2}, \\
\sigma_{\mathrm{EVPA}}=\frac{\sqrt{Q^{2} \sigma_{U}^{2}+U^{2} \sigma_{Q}^{2}}}{2\left(Q^{2}+U^{2}\right)}=\frac{\sigma_{p}}{2 p},
\end{gathered}
$$

and

$$
\sigma_{m}=\frac{\sigma_{p}}{I}
$$




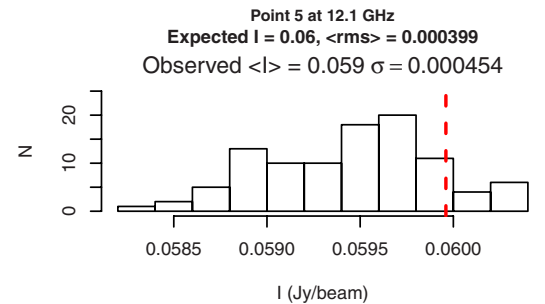

T(Jy/beam)
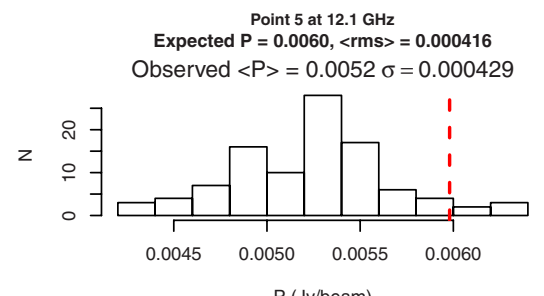

$\mathrm{P}(\mathrm{Jy} / \mathrm{beam})$

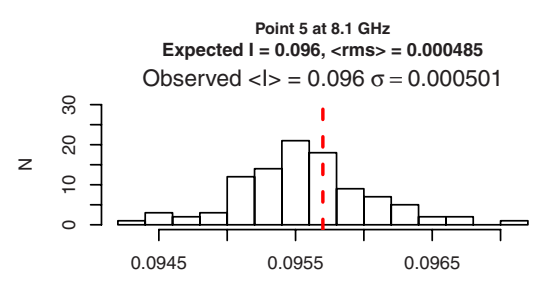

I (Jy/beam)

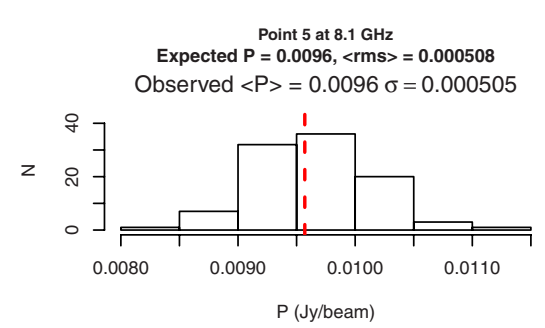

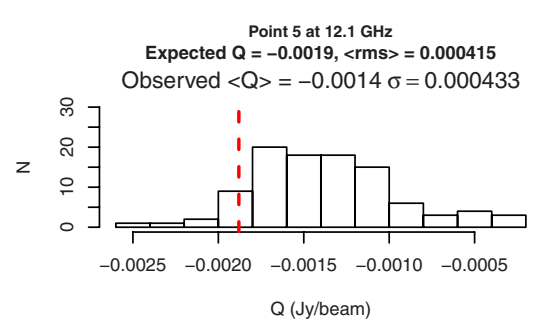
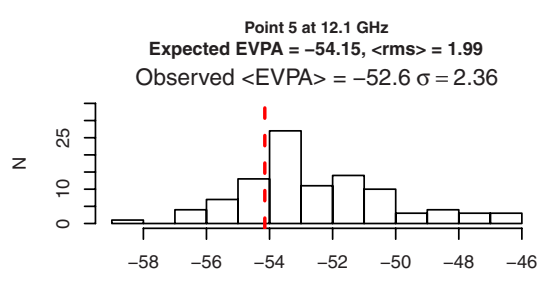

EVPA (deg.)

Point 5 at $8.1 \mathrm{GHz}$

Expected $Q=-0.0030,<\mathrm{rms}>=0.000505$

Observed $\langle Q\rangle=-0.003 \sigma=0.000518$

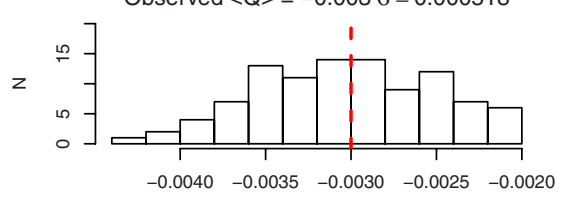

Q (Jy/beam)

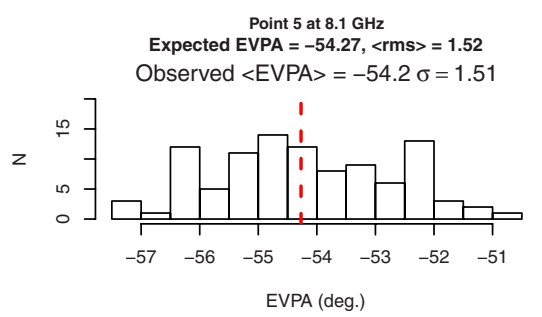

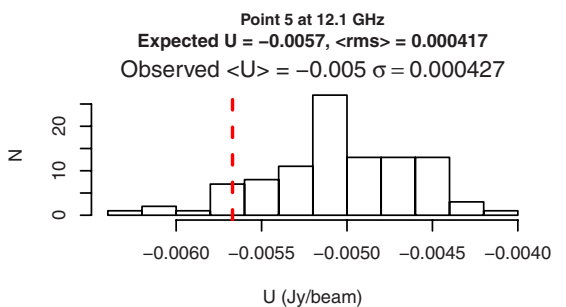

(Jy/beam)

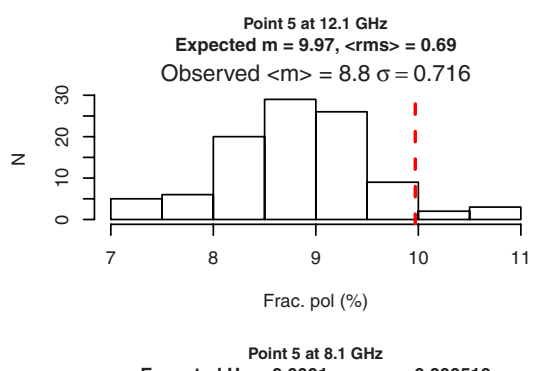

Expected $\mathrm{U}=-0.0091,<\mathrm{rms}>=0.000510$ Observed $\langle U\rangle=-0.0091 \sigma=0.000497$

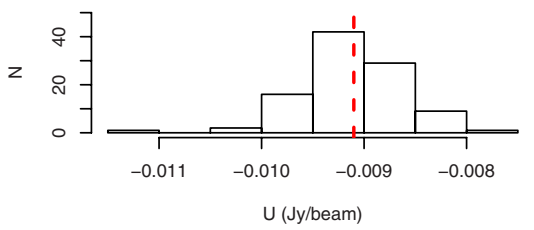

Point 5 at $8.1 \mathrm{GHz}$

Expected $m=10.0,<\mathrm{rms}>=0.76$

Observed $<\mathrm{m}>=10.1 \sigma=0.529$

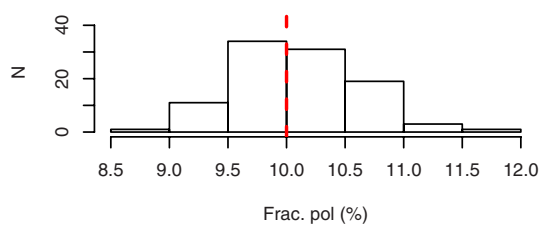

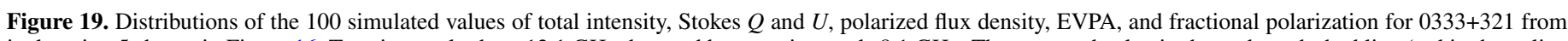

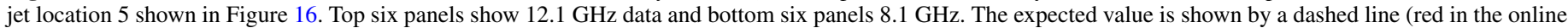
journal). See the text for information on the expected error values.

(A color version of this figure is available in the online journal.)

where $\sigma_{Q}$ and $\sigma_{U}$ are the $Q$ and $U$ rms values in most of the simulations, and $Q$ and $U$ are the Stokes parameters in the given pixel. In Equation (B3) we assume that the $\sigma_{I}$ term can be neglected as $\left(p / I^{2}\right)^{2}$ will be very small for all our components. For the simulations that take the instrumental polarization into account, an additional error $\sigma_{\text {Dterm }}$ defined as

$$
\sigma_{\text {Dterm }}=\frac{0.002}{\left(N_{\text {ant }} \times N_{\mathrm{IF}} \times N_{\text {scan }}\right)^{1 / 2}}\left(I^{2}+\left(0.3 \times I_{\text {peak }}\right)^{2}\right)^{1 / 2},
$$

where $N_{\text {ant }}$ is the number of antennas, $N_{\text {IF }}$ is the number of IFs, $N_{\text {scan }}$ is the number of scans with independent parallactic angles, and $I_{\text {peak }}$ is the peak total intensity of the map (Roberts et al. 1994). This additional error term is added in quadrature to the rms errors. The factor 0.002 in the equation is determined from the scatter of the D-terms in our data. In our data the number of antennas is 10 , the number of IFs is 4 for 15.4 and $12.1 \mathrm{GHz}, 2$ for 8.4 and $8.1 \mathrm{GHz}$, and the number of independent scans is 4 . The equation is defined and explained by Roberts et al. (1994), where they study the effects of instrumental polarization with detailed simulations. For example, they show that the D-term errors scatter across the image (factor 0.3 in Equation (B4)) which seems to be supported by our simulations. Therefore, we include a contribution from the total intensity peak and the current location in Equation (B4).

The D-term error is strongly dependent on the Stokes $I$ and is not distributed evenly across the images. Therefore, its effect is largest on the bright locations of the source, especially near the core and it is not accounted for in the rms errors. To demonstrate the effect, in Figure 17 we show distributions of total intensity $I$, Stokes parameters $Q$ and $U$, polarized flux density $p$, EVPA and fractional polarization $m$ for $1226+023$ at point 0 (shown by a star) of Figure 15. In this simulation $Q$ and $U$ were set to be $0.0707 \times I$ in each component. Without any noise added, this results in uniform fractional polarization $m=0.1$ and EVPA $=-22.5$. Above each histogram we give the expected value for each parameter and its expected error value calculated using Equations (B1), (B2), and (B3). The top six panels show 100 simulations without adding the D-term error in step 5 in the simulations. As can be seen from the expected and observed values, the observed standard deviations of $Q$ and $U$ are slightly larger than the measured $Q_{\mathrm{rms}}$ and $U_{\mathrm{rms}}$ and the distributions are not peaking at the expected values, which can be attributed to the CLEAN errors. In the bottom six panels, the simulation is repeated with additional D-term noise added, which is also included in the expected error value using Equation (B4). Now 


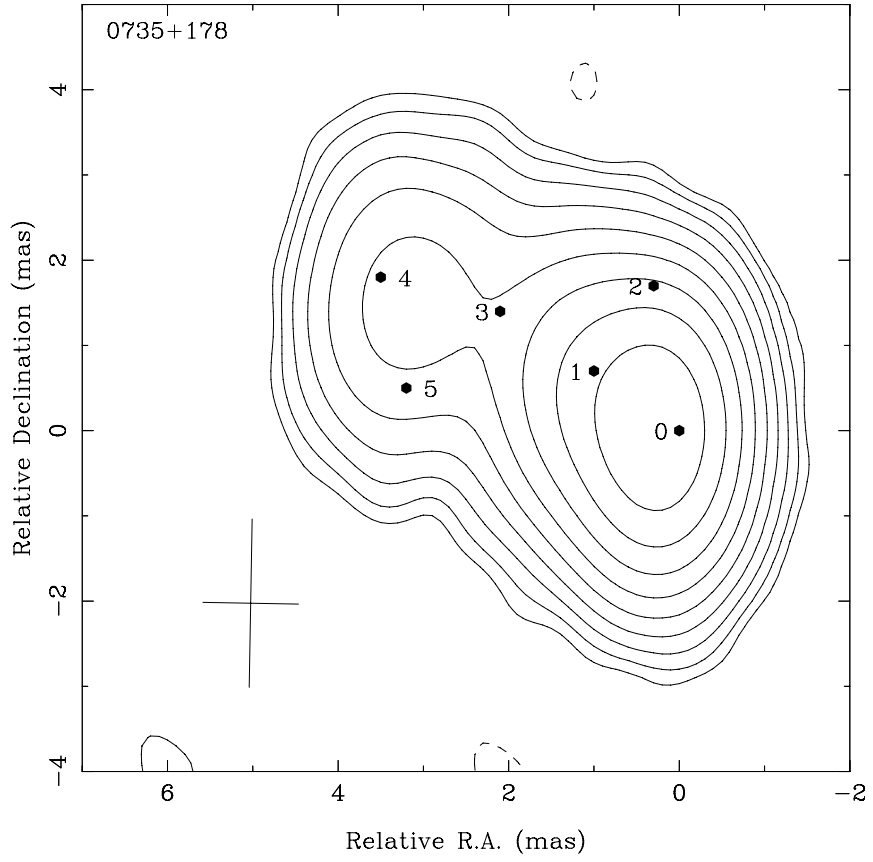

Figure 20. Locations of the simulated RM distributions in $0735+178$ overlaid on the $15 \mathrm{GHz}$ total intensity contours. the expected and observed rms values correspond very well to each other. In this bright location of the source, the contribution from D-term errors to the error in polarized flux density is twice as large as the rms error ( $1.1 \mathrm{mJy}$ compared to $0.56 \mathrm{mJy}$ ). The distributions of other components are not shown here but they follow the same pattern, except that the D-term error contribution diminishes with decreasing Stokes $I$ so that in most of the components of 3C 273 the rms errors and D-term errors are of the same order. In fainter sources, such as $0333+321$ the D-term errors are typically negligible in the jet components and smaller than rms errors even in the core. This effect is correctly accounted for by using Equation (B4).

In order to study the effects of $(u, v)$-clipping and the CLEAN procedure, we simulated the original $15 \mathrm{GHz}$ data of $0333+321$ and the $(u, v)$-clipped data restored to the $8.1 \mathrm{GHz}$ beam. In these simulations we set $Q=-0.031623 \times I$ and $U=-0.094869$ $\times I$, which results in uniform fractional polarization $m=0.1$ and EVPA $=-54.217$. The expected values were determined as in the case of $1226+023$. In Figure 18 in the top six panels we show the distributions for the original images and in the bottom six panels the distributions for the images that have a reduced $(u, v)$-coverage corresponding to the real data and restored with the $8.1 \mathrm{GHz}$ beam size. These are taken at location 6 (shown by a star) in Figure 16 to show the effects on a faint

Point $0:<\mathrm{l}>=9.64<$ RMe $>=70.9$

Point 1: $<\mathrm{l}>=4.33<\mathrm{RMe}>=70.9$

Point 2: $\langle l>=0.68<$ RMe $>=71.0$

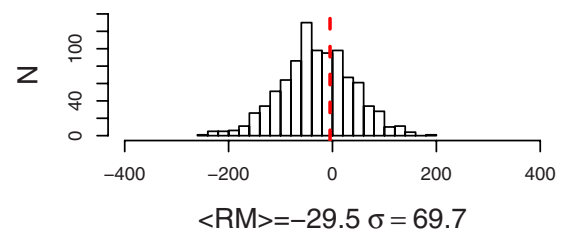

Point 3: $<\mathrm{l}>=2.33<\mathrm{RMe}>=70.9$

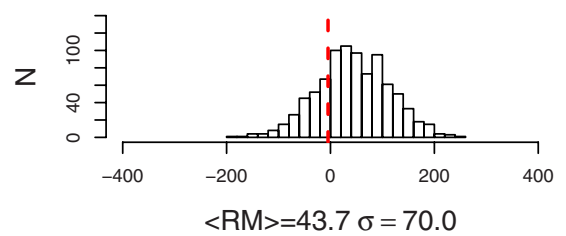

Point 6: $<\mathrm{l}>=0.03<\mathrm{RMe}>=86.5$

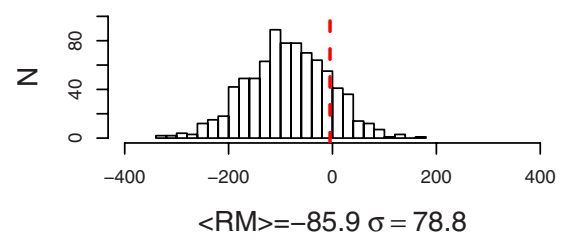

Point 9: $<\mathrm{l}>=0.05<$ RMe $>=77.2$

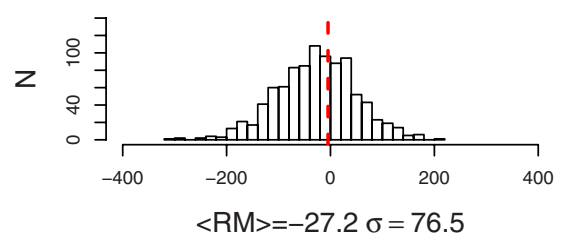

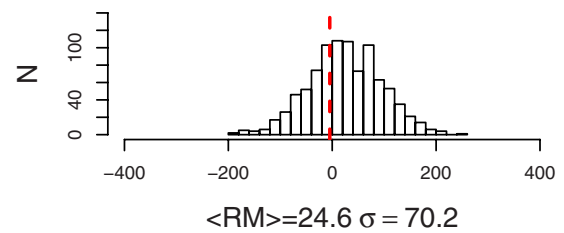

Point 4: $\langle\mathrm{l}>=0.28<\mathrm{RMe}>=71.4$

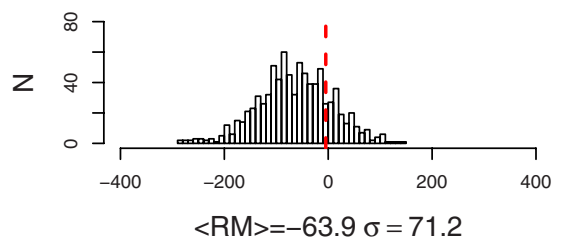

Point 7: $<\mathrm{l}>=0.98<\mathrm{RMe}>=71.0$

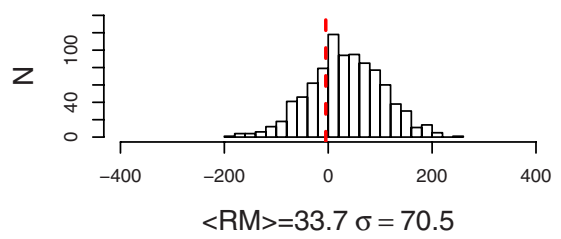

Point 5: $<\mathrm{l}>=0.60<\mathrm{RMe}>=71.0$

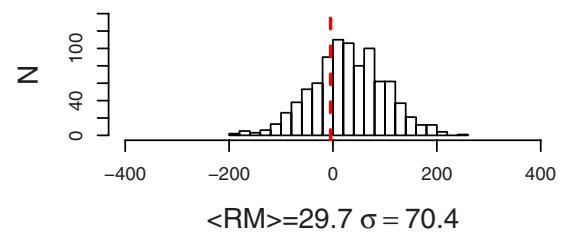

Point 8: $<1>=0.06<$ RMe $>=75.7$

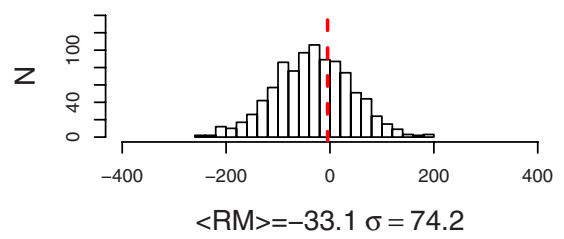

Figure 21. Distributions of the 1000 simulated RM values for $1226+023$ from jet locations shown in Figure 15 . Average total intensity value $\langle I\rangle$, average RM error from the variance-covariance matrix $\langle\mathrm{RMe}\rangle$, average $\mathrm{RM}$ value $\langle\mathrm{RM}\rangle$ from the simulated jets, and the standard deviation of the distribution are given for each location. The expected RM value is shown by a dashed line (red in the online journal).

(A color version of this figure is available in the online journal.) 
Point 0: $\langle l>=0.97<R M e>=71.0$

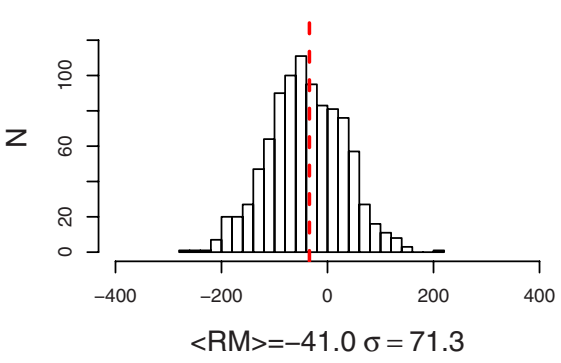

Point 3: $\langle\mathrm{l}>=0.07<\mathrm{RMe}>=75.6$

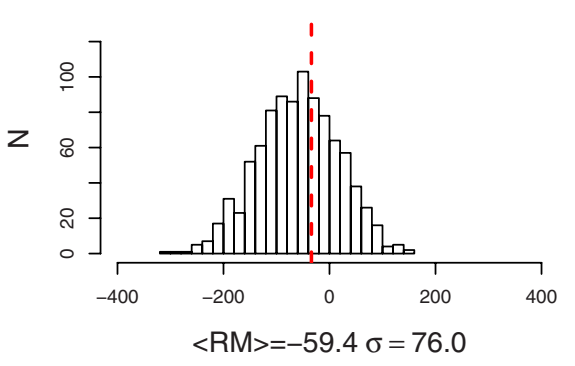

Point 6: $\langle\mathrm{l}>=0.04<\mathrm{RMe}>=87.1$

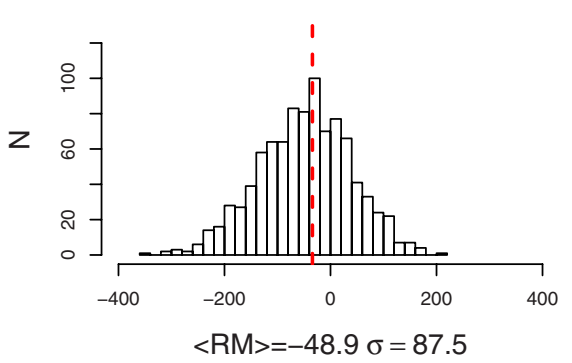

Point 1: $<\mathrm{l}>=\mathbf{0} .41<\mathrm{RMe}>=71.1$

Point 2: $<\mathrm{l}>=0.09<\mathrm{RMe}>=73.9$

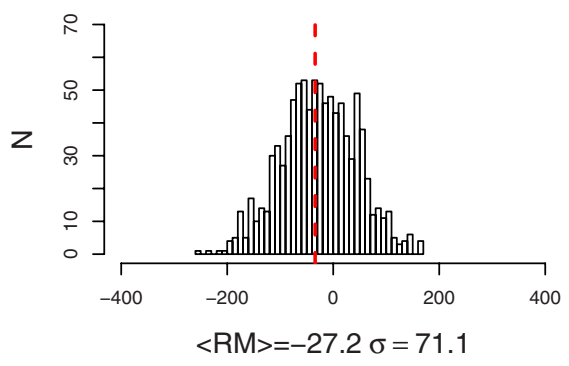

Point 4: $<\mathrm{l}>=0.05<\mathrm{RMe}>=81.9$

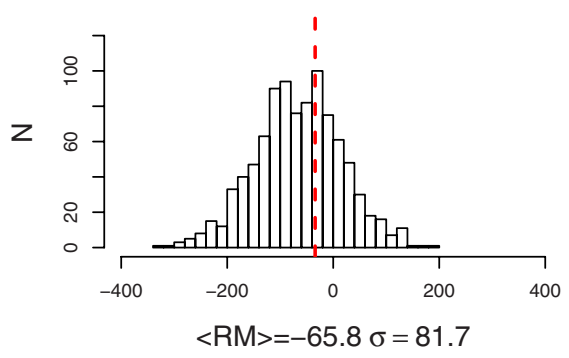

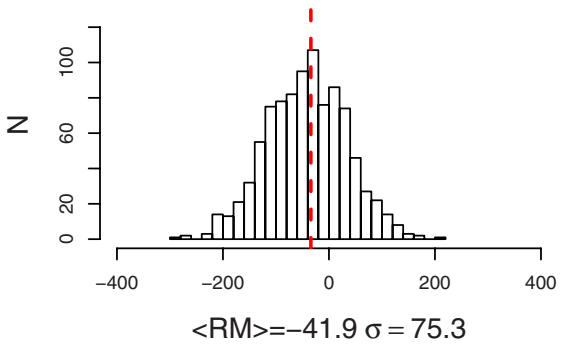

Point 5: $<\mathrm{l}>=0.03<\mathrm{RMe}>=89.5$

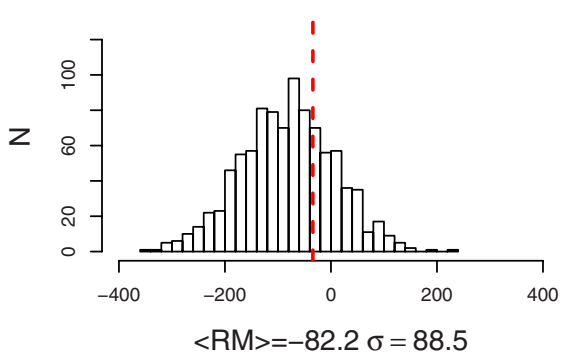

Figure 22. Distributions of the 1000 simulated RM values for $0333+321$ from jet locations shown in Figure 16 . Average total intensity value $\langle I\rangle$, average RM error from the variance-covariance matrix $\langle\mathrm{RMe}\rangle$, average $\mathrm{RM}$ value $\langle\mathrm{RM}\rangle$ from the simulated jets, and the standard deviation of the distribution are given for each location. The expected RM value is shown by a dashed line (red in the online journal).

(A color version of this figure is available in the online journal.)

edge region. Our equations for the error values work very well, showing that the CLEAN procedure does not affect the standard deviation of the distributions, but there is a large offset in the peaks of the distributions in both simulations. This is also seen in the lower frequency simulations, for which we show an example at 12.1 and $8.1 \mathrm{GHz}(8.4 \mathrm{GHz}$ is nearly identical to $8.1 \mathrm{GHz}$ and therefore not shown) in Figure 19. This is due to the reduced $(u, v)$-coverage, especially at 15.4 and $12.1 \mathrm{GHz}$, and the CLEAN procedure. By looking at the offsets in all the component locations in the different sources and all the frequency bands, we find the error to vary between 1 and 3 times the rms error and therefore we set it to be 1.5 times the rms error in our error estimates.

Based on all these simulations, we conclude that the errors in polarized flux density, EVPA and fractional polarization should be calculated using Equations (B1), (B2), and (B3), where $\sigma_{Q}$ and $\sigma_{U}$ should include contributions from the rms error, D-term error and CLEAN error so that they are defined as

$$
\sigma=\left(\sigma_{\mathrm{rms}}^{2}+\sigma_{\text {Dterm }}^{2}+\left(1.5 \times \sigma_{\mathrm{rms}}\right)^{2}\right)^{1 / 2},
$$

where $\sigma_{\text {rms }}$ is the corresponding rms error and $\sigma_{\text {Dterm }}$ is calculated using Equation (B4). The errors for our analysis are defined using the above criteria.

\section{APPENDIX C ERRORS IN RM MAPS}

Errors in the RM values can formally be obtained from the variance-covariance matrix of the linear fit as the error of the slope. This method is used in the RM task in AIPS. The resulting errors depend largely on the error of the EVPA, which is dominated by the absolute calibration error in the bright jet locations. As we show in Appendix B, the rms contribution of the EVPA error is well-described by error propagation from $Q$ and $U$ rms values. It is crucial to obtain a correct error estimate for the RM values in order to distinguish between various depolarization models and to properly study gradients in the RM within a source. Using the same kind of approach as in Appendix B, we simulated the effect of random noise and calibration error to see if the error estimate from the variance-covariance matrix is correct.

The simulations were done in the same manner as in Appendix B but repeating the procedure for all the frequency bands. We then added a random error drawn from a Gaussian distribution with standard deviation corresponding to the calibration error at the given frequency band to each of the EVPA images. This error is added systematically to each pixel in a 
Point $0:<l>=0.44<R M e>=71.3$

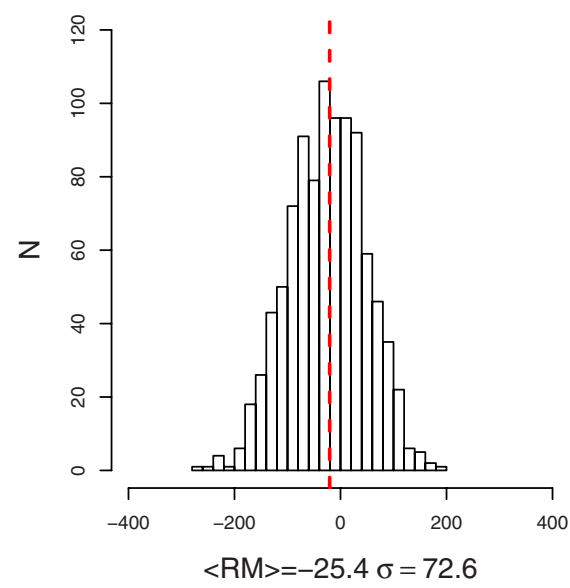

Point 3: $<1>=0.04<\mathrm{RMe}>=86.9$

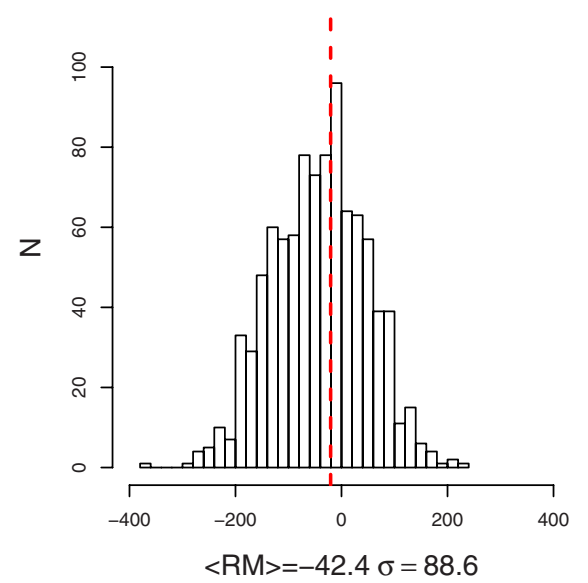

Point 1: $<\mathrm{l}>=0.27<\mathrm{RMe}>=71.5$

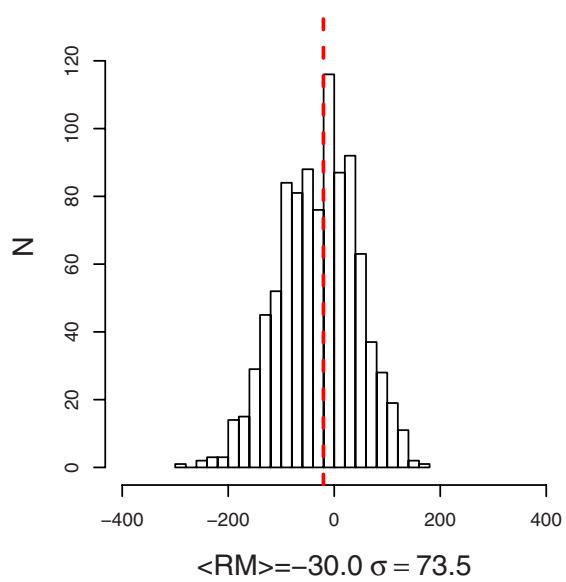

Point 4: $<\mathrm{l}>=0.04<\mathrm{RMe}>=86.3$

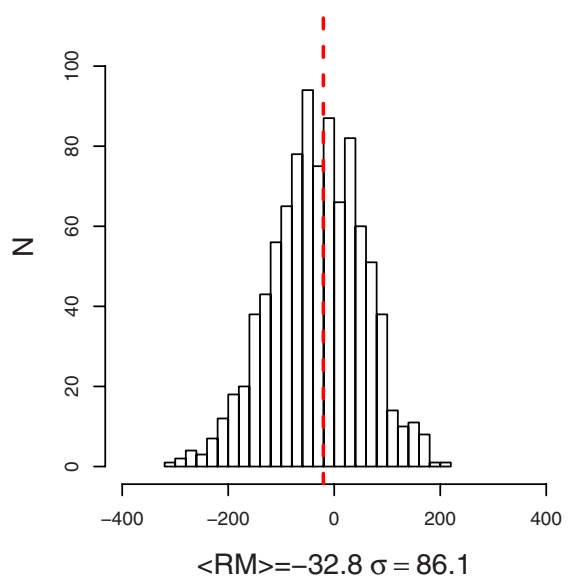

Point 2: $\langle\mathrm{l}>=0.06<\mathrm{RMe}>=\mathbf{8 1 . 0}$

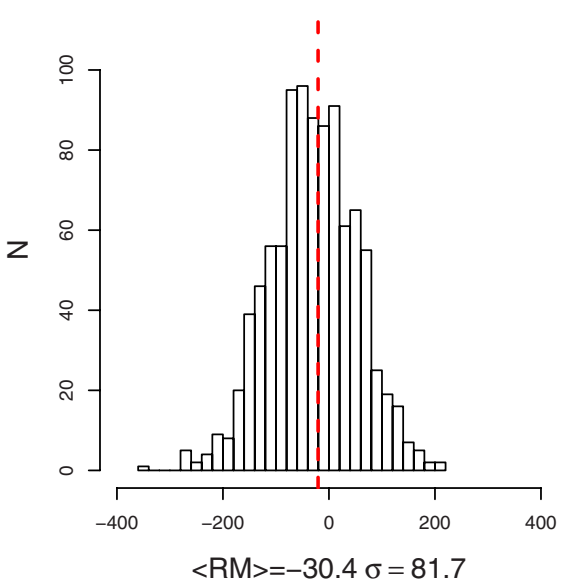

Point 5: $<\mathrm{l}>=0.03<\mathrm{RMe}>=98.3$

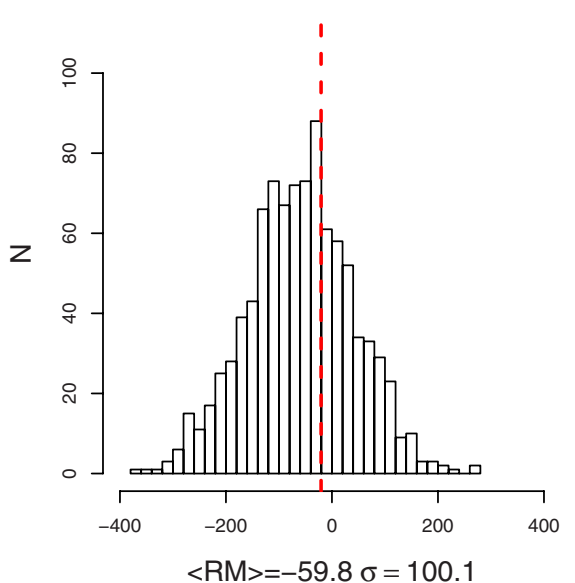

Figure 23. Distributions of the 1000 simulated RM values for $0735+178$ from jet locations shown in Figure 20. Average total intensity value $\langle I\rangle$, average RM error from the variance-covariance matrix $\langle\mathrm{RMe}\rangle$, average $\mathrm{RM}$ value $\langle\mathrm{RM}\rangle$ from the simulated jets, and the standard deviation of the distribution are given for each location. The expected RM value is shown by a dashed line (red in the online journal).

(A color version of this figure is available in the online journal.)

given image but varies randomly from one epoch to another and needs to be accounted for in order to obtain correct error estimates for the RM. The simulated RM maps were then created using the same script as for the real data and 1000 RM maps were obtained. Without any added noise, the expected value for the $\mathrm{RM}$ in each map is $0 \mathrm{rad} \mathrm{m}^{-2}$ minus any Galactic Faraday rotation. This is because we created the simulated maps using the exact same procedure as for the real data and therefore also the Galactic Faraday rotation correction was taken into account.

We repeated the simulations for three sources, $0333+321$, $0735+178$, and $1226+023$. The source $0735+178$ was included so that we could study the effect on a faint source which does not have a well-resolved jet. The expected value of RM in each source is different because of the differing Galactic Faraday rotation. For $0333+321$ we expect an RM of $-34.1 \mathrm{rad} \mathrm{m}^{-2}$, for $0735+178$ an $\mathrm{RM}$ of $-20.5 \mathrm{rad} \mathrm{m}^{-2}$, and for $1226+023$ an RM of $-4.7 \mathrm{rad} \mathrm{m}^{-2}$.

\section{C.1. Errors in Individual Jet Locations}

In order to study the RM error in individual jet locations, we chose the same jet locations for $0333+321$ and $1226+023$ as in Appendix B and shown in Figures 15 and 16. For 0735+178 we chose the locations shown in Figure 20. The distributions of the 1000 simulated values are shown in Figures 21-23. Once again it is clear that the standard deviations $\sigma$ from our simulations, listed below each distribution, agree well with the error from the variance-covariance matrix $\langle\mathrm{RMe}\rangle$ (average value over the 1000 simulations), listed above each distribution. There is, however, a visible offset in the peaks of the distributions to the left of the expected value. This offset is due to small errors in the final CLEAN procedure as shown in Appendix B and it is accounted for by the additional error due to the CLEAN procedure, which is taken into account in our final $Q$ and $U$ errors.

Our simulations also show that it is common to obtain very high $\mathrm{RM}$ values of $\sim \pm 2 \times 10^{4} \mathrm{rad} \mathrm{m}^{-2}$ purely due to noise in the data. An example of such a simulated jet is shown in Figure 24. We find at least one very high RM pixel in $67 \%$ of the simulated maps in $0735+178$, and in $74 \%$ in $0333+321$ and in all the simulated maps of $1226+023$. The number of the highRM pixels depends on the total number of pixels in the simulated maps. In $1226+023$ the fraction is much higher because it has five times more pixels than the two other sources. The median fraction of high-RM pixels in the simulated maps is $0.3 \%-0.6 \%$ depending on the source but in $5.5 \%-26 \%$ of the maps there are more than 100 such pixels, resulting in patches of at least 


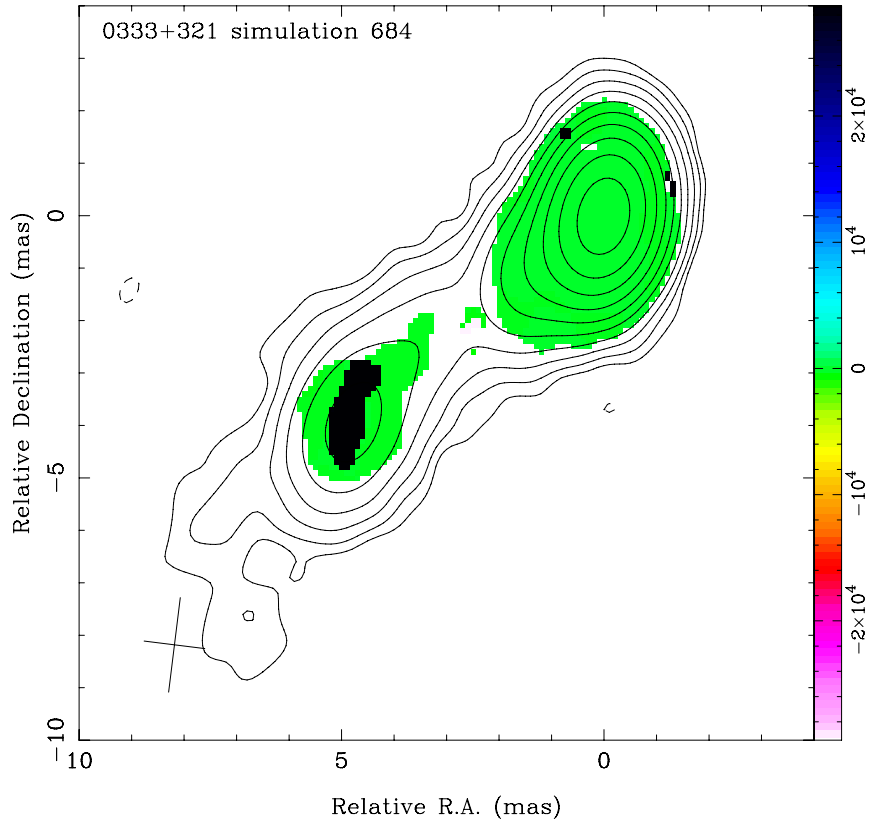

Figure 24. Example of a simulation in which extreme RM values are generated due to noise in the data.

(A color version of this figure is available in the online journal.)

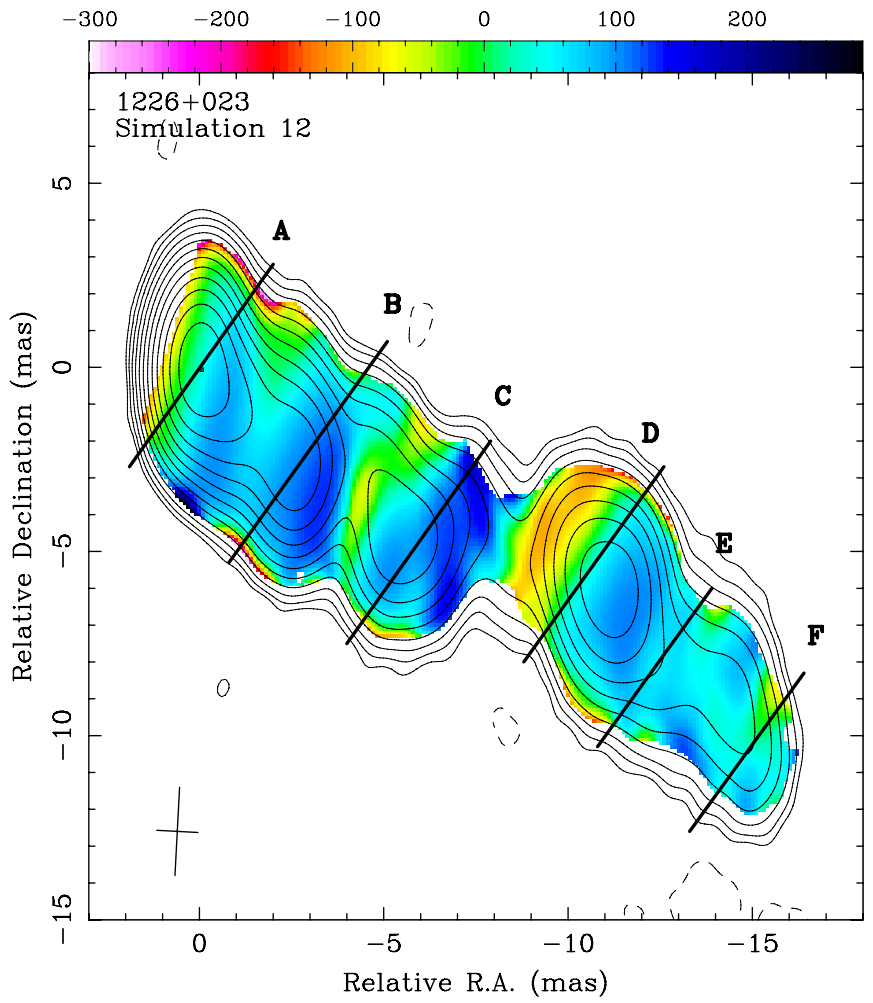

Figure 25. Slices transverse to the jet in $1226+023$ overlaid on a simulated RM map.

(A color version of this figure is available in the online journal.)

$1 \times 1$ mas. This happens because the pixels are not independent on scales smaller than the beam size. Therefore, even fairly large very high-RM regions like these in the real RM maps are most likely spurious. For this reason, in our real RM maps, we have blanked the high-RM pixels because they are most likely due to noise in the data and do not represent real structure.

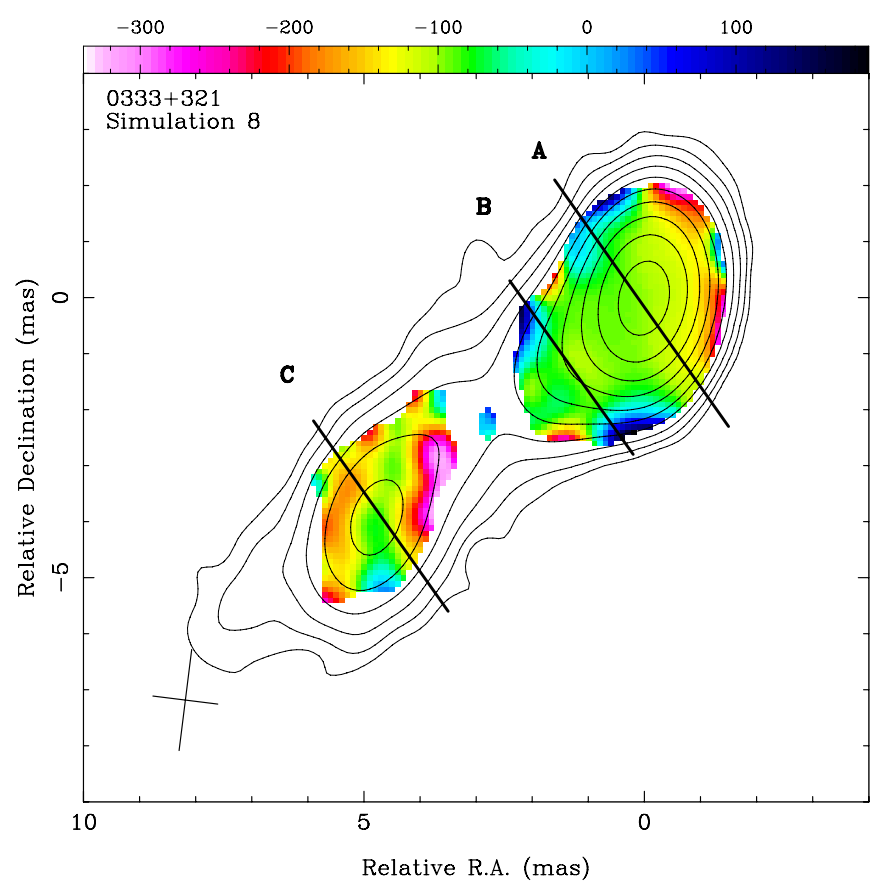

Figure 26. Slices transverse to the jet in $0333+321$ overlaid on a simulated RM map.

(A color version of this figure is available in the online journal.)

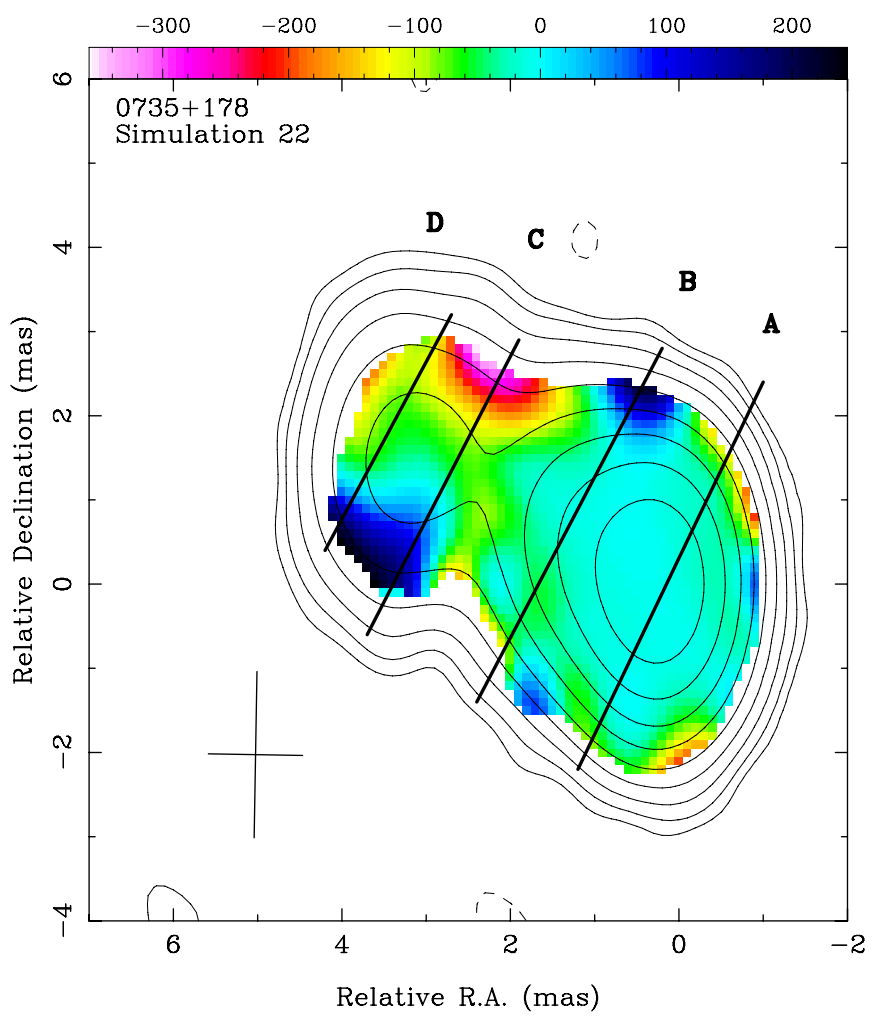

Figure 27. Slices transverse to the jet in $0735+178$ overlaid on a simulated RM map.

(A color version of this figure is available in the online journal.)

Based on the simulations we conclude that the error from the variance-covariance matrix of the linear fit is a good estimate for the true error in the RM in individual jet locations. 
$1226+023$ slice $B$

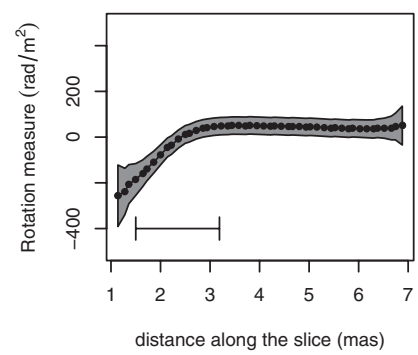

$1226+023$ slice $F$

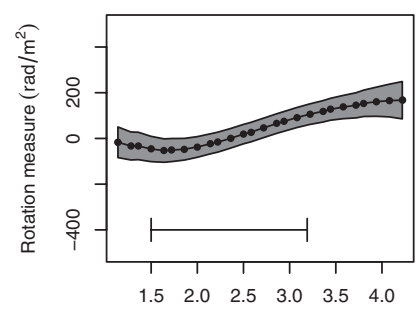

distance along the slice (mas)

0735+178 slice A

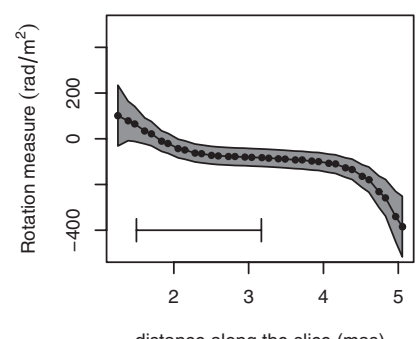

$1226+023$ slice $C$

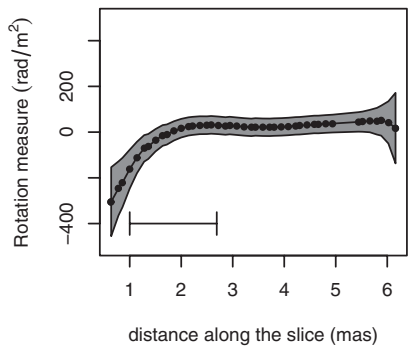

0333+321 slice A

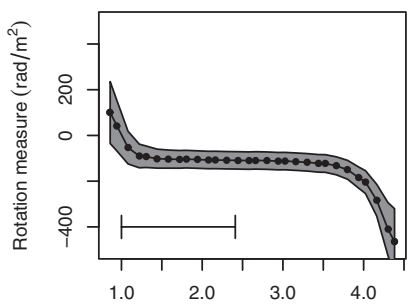

distance along the slice (mas)

0735+178 slice B

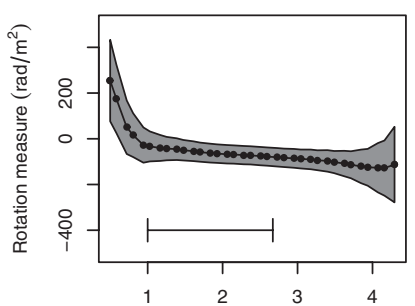

distance along the slice (mas)
$1226+023$ slice D

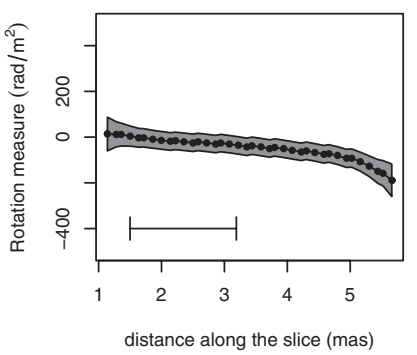

0333+321 slice B

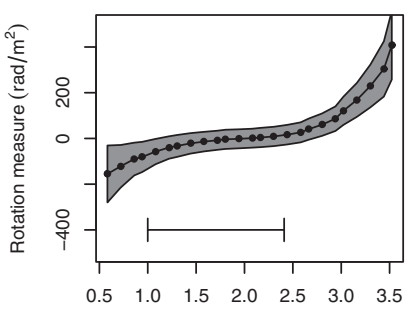

distance along the slice (mas)

0735+178 slice C

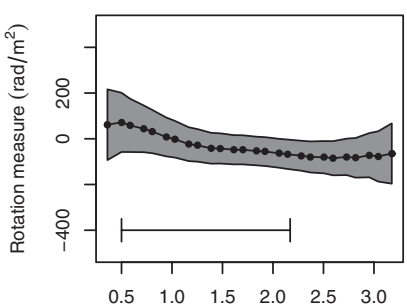

distance along the slice (mas)
$1226+023$ slice $E$

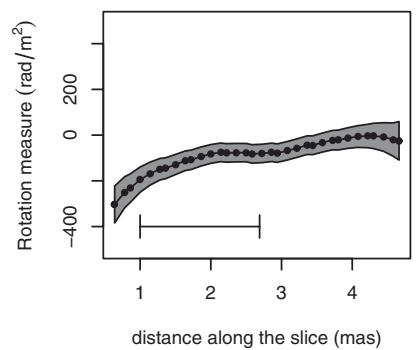

0333+321 slice C

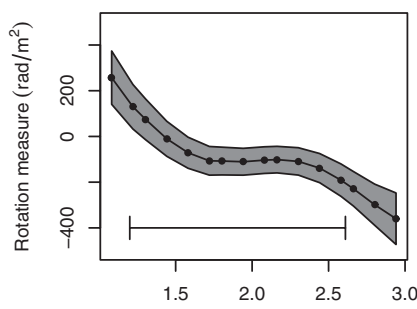

distance along the slice (mas)

0735+178 slice D

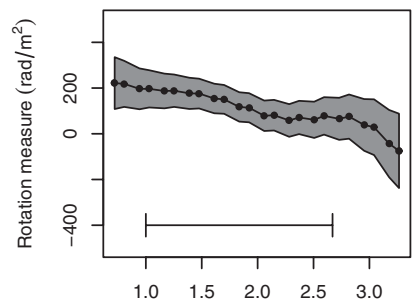

distance along the slice (mas)

Figure 28. Most extreme examples of simulated transverse gradients over the slices across the jet locations shown in Figures 25-27. None of these simulated jets contain an actual RM gradient. The FWHM beam size along the slice direction is shown in each plot as a scale bar.

\section{C.2. Errors in RM Gradients}

Over the past decade there have been several claims of detections of RM gradients in many AGNs (see, e.g., Contopoulos et al. 2009 for a compilation of results). These results are still somewhat controversial due to the difficulty in addressing the errors in the gradients (Taylor \& Zavala 2010). Murphy \& Gabuzda (2012) convolve simulated gradients with different beam sizes and argue that a gradient can be significant even when the jet is not resolved. Their simulation does not, however, take into account noise in the data. With simulations we have shown that the errors in individual pixels are well described by the error from the variance-covariance matrix of the linear fit. It is more complicated to study the errors in the RM gradients, or more precisely, the probability of detecting a spurious RM gradient due to noise in the data. Several of our simulated jets show structures resembling real gradients primarily due to effects of the finite beam size. To quantify this effect, we have taken several transverse slices across the simulated jets, shown in Figures 25-27, and fitted a simple line to the data. We have then looked at the distributions of the slopes, which give us the maximum spurious gradient in rad $\mathrm{m}^{-2} \mathrm{mas}^{-1}$, created by noise in the data.

In Figure 28 we show examples of the slices in the different sources. One thing to note is that when studying gradients, the absolute EVPA calibration error can be ignored because it affects each pixel in the same direction and thus will not affect a gradient across the same source (Mahmud et al. 2009). Therefore, in each pixel we have subtracted in quadrature the amount of calibration error, $\sim 60 \mathrm{rad} \mathrm{m}^{-2}$, from the total RM error. It is very important to note that the pixels are not independent but very much affected by the beam size. We have plotted the size of the beam along the transverse slice in each plot to show the scale in which the pixels are not independent. As can be seen from the plots, depending on the slice, there are only one to four independent points along each slice.

The distributions of the measured slopes are shown in Figure 29. In the calculation of the slope we have ignored the very high RM values. This resulted in slices with only a few pixels with measured RMs which usually resulted in steep slopes. These are ignored in the distribution plots. The most notable are the distributions from slices C in $0333+321$ and slices D in $0735+178$ which show gradients up to $200 \mathrm{rad} \mathrm{m}^{-2} \mathrm{mas}^{-1}$. The slices are about 1.5 beam sizes wide and with a typical beam width along the slice of 1.5 mas, this would result in a total gradient of $450 \mathrm{rad} \mathrm{m}^{-2}$ over the slice. This is of the same order as most of the gradients in the literature. It is very close to the value we see in the jet of 3C 273. Reassuringly, the magnitude of the spurious gradient is strongly dependent on the width of the jet with respect to the beam size as shown in 
$1226+023$ slice $\mathrm{B}: \sigma=8.6$

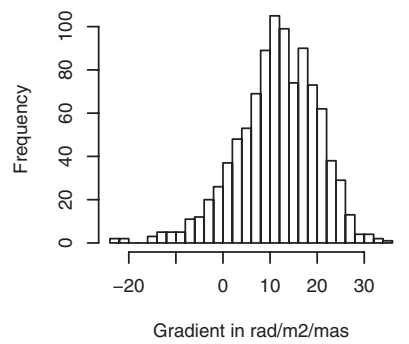

1226+023 slice F: $\sigma=20.8$

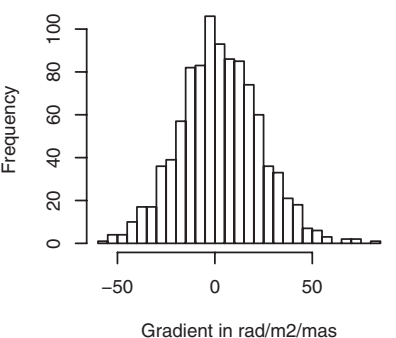

$0735+178$ slice A: $\sigma=21.4$

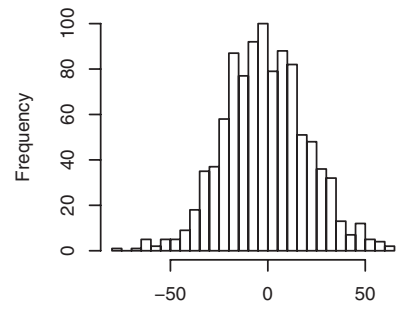

Gradient in $\mathrm{rad} / \mathrm{m} 2 / \mathrm{mas}$
1226+023 slice C: $\sigma=9.9$

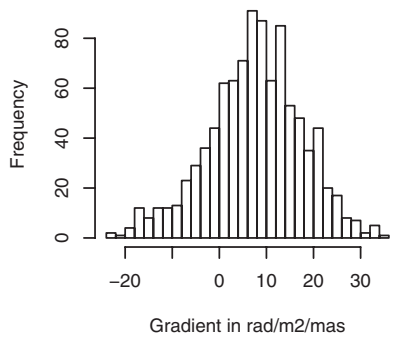

0333+321 slice A: $\sigma=21.4$

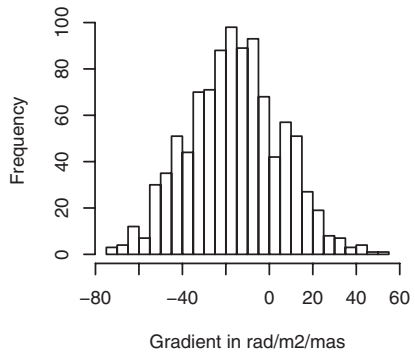

$0735+178$ slice B: $\sigma=30.1$

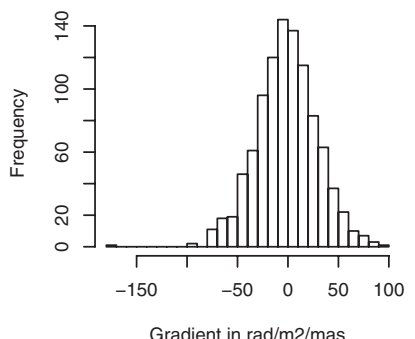

$1226+023$ slice D: $\sigma=7.7$

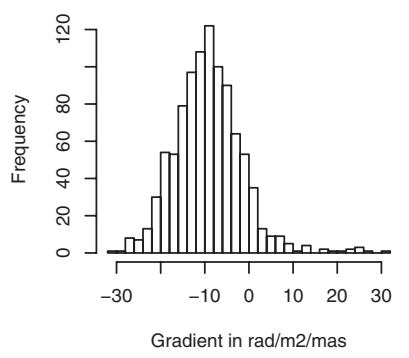

0333+321 slice B: $\sigma=36.3$

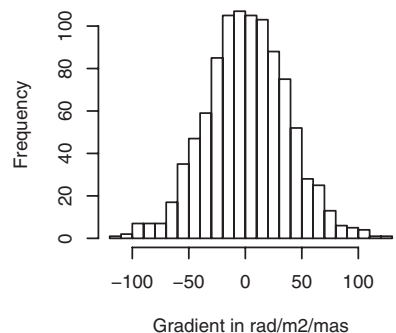

$0735+178$ slice C: $\sigma=40.9$

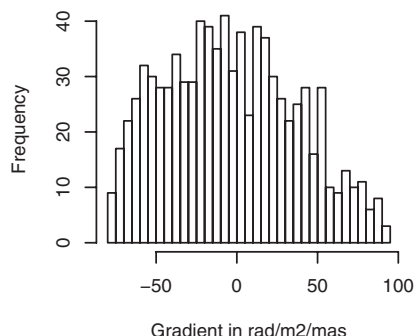

1226+023 slice $\mathrm{E}: \quad \sigma=14.3$

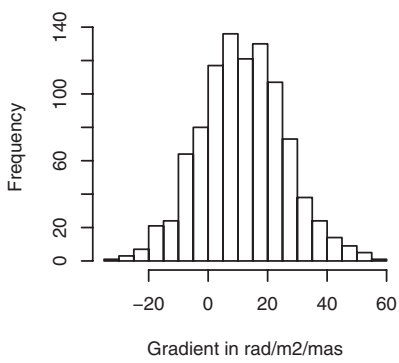

0333+321 slice C: $\sigma=65.4$

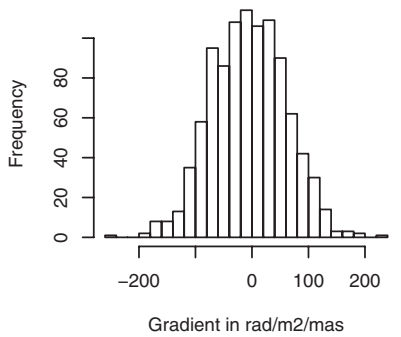

$0735+178$ slice $\mathrm{D}: \sigma=59.7$

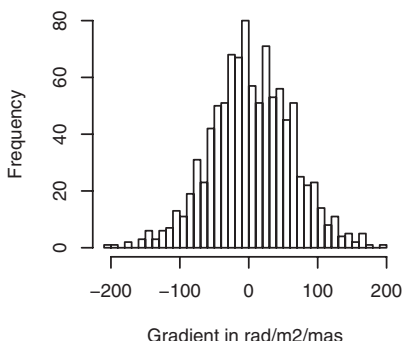

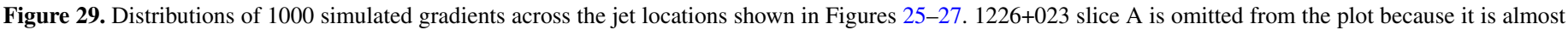
identical to slice B.

Figure 30 (left panel). When the width of the polarized jet approaches three beam widths, the spurious gradient diminishes. To calculate the jet width in beams we first take the width of the slice in pixels and divide that by the beam size along the slice direction in pixels. This is because we want to ensure that we take into account that we do not detect polarization in as large area as total intensity and require the jet to be resolved in polarization as well.

We also note that almost all the spurious gradients we see in the simulations are smaller than three times the RM errors at the location of the slice if using the errors as we have defined them and therefore would not be accepted as true gradients in our real data. This is shown in Figure 30 (right panel), where we plot the fraction of false positives, i.e., number of spurious gradients which exceed the error limit, against the jet width for $1 \sigma, 2 \sigma$, and $3 \sigma$ limits. Here, $\sigma$ is defined as the largest error bar in the end of a gradient slice. This plot clearly demonstrates how the fraction of false positives goes to zero if the jet is two beams wide and a $3 \sigma$ error limit is used. When the jet is more than 2.5 beams wide, even a $2 \sigma$ limit might be sufficient, if additionally taking into account the magnitude of the maximum possible spurious gradient shown in the left panel. For our specific fourfrequency setup between 8 and $15 \mathrm{GHz}$ using $128 \mathrm{Mbits} \mathrm{s}^{-1}$ recording bit rate and $\sim 60$ minute on-source time, we find the limit to be $200 \mathrm{rad} \mathrm{m}^{-2} \mathrm{mas}^{-1}$.
Our simulations also verify that it is impossible to get a persistent gradient over the whole jet length, as is seen in 3C 273, due to noise in the data. Therefore, it is important that if gradients are detected, they are seen in multiple jet locations more than a beam width apart, or in the same jet location over multiple epochs, and that the jet is well-resolved in polarized flux density, preferably over two times the beam width. When studying jets that are not wide enough, our simulation results can be used as "rules of thumb" for estimating the magnitude of a reliable gradient. For example, in a jet that is two beams wide, it is possible to have spurious gradients up to $100 \mathrm{rad} \mathrm{m}^{-2} \mathrm{mas}^{-1}$ which would result in a total gradient of $300 \mathrm{rad} \mathrm{m}^{-2}$ if the beam width is 1.5 mas. We note that detection of three $2 \sigma$ gradients at different epochs corresponds to a $3 \sigma$ detection so that multiepoch observations can help to determine if observed gradients in jets that are not well resolved are real. However, this requires correct treatment of the RM errors and is not recommended for jets less than 1.5 beams wide due to the large fraction of false positives at these jet widths.

Our simulations overall agree well with the suggestions by Taylor \& Zavala (2010) to consider a gradient reliable if the jet is more than three beams wide and use $3 \sigma$ error limits. Based on our simulations a jet that is two beams wide is already sufficient if the gradient exceeds the $3 \sigma$ limit. Attempting to detect RM gradients in jets less than two beams wide is discouraged as 

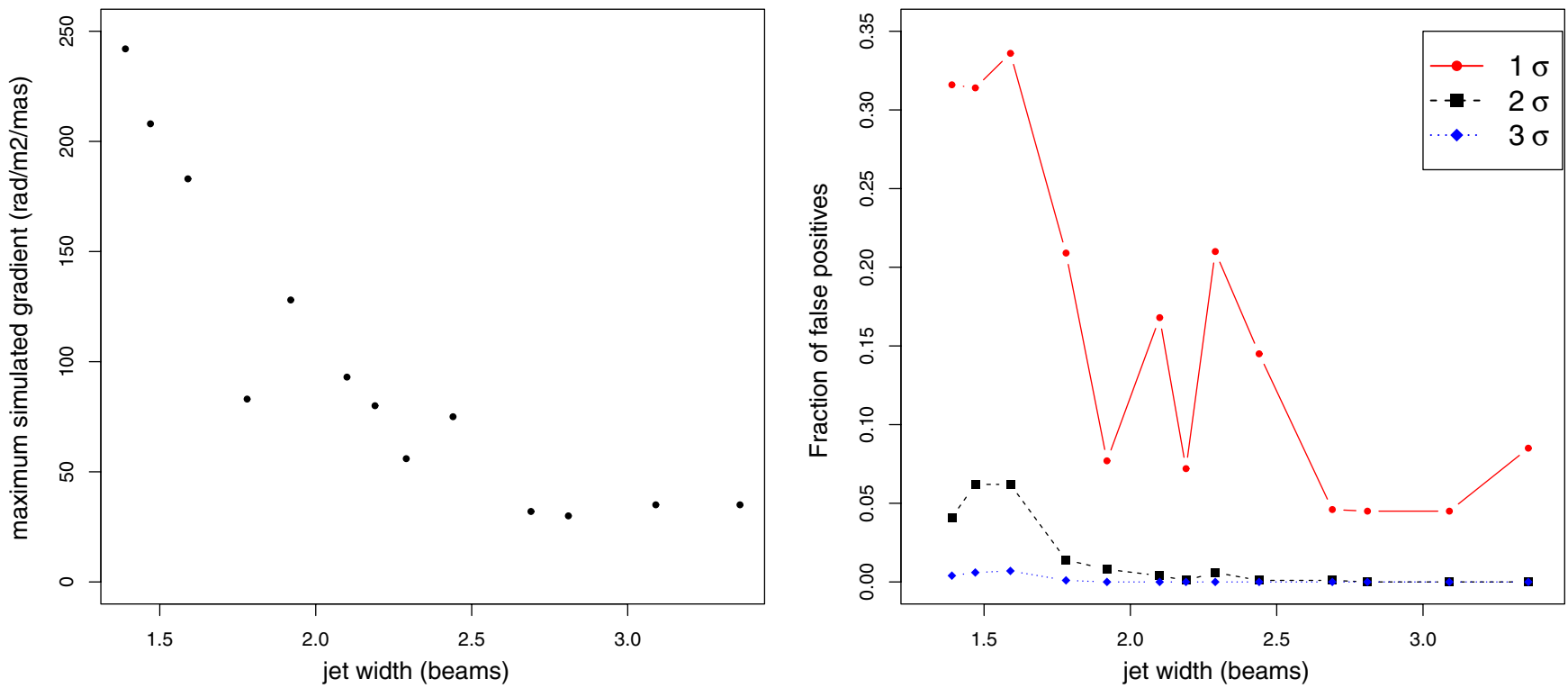

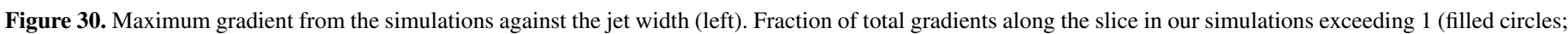
red in the online journal), 2 (filled squares), or 3 (filled diamonds, blue in the online journal) standard deviations against the jet width.

(A color version of this figure is available in the online journal.)

the probability to detect false positives exceeds 0.02 even when a $3 \sigma$ limit is used. We emphasize that this approach requires the RM errors to be calculated from the variance-covariance matrix of the EVPA versus $\lambda^{2}$-fit with appropriate EVPA errors taken into account; calibration errors can then be subtracted from the error bars. These simulations are applicable to the four-frequency configuration used in this paper and should be repeated if different frequency bands are used.

\section{REFERENCES}

Alberdi, A., Goméz, J.-L., Marcaide, J. M., Marscher, A. P., \& Pérez-Torres, M. A. 2000, A\&A, 361, 529

Algaba, J. C., Gabuzda, D. C., \& Smith, P. S. 2011, MNRAS, 411, 85

Asada, K., Inoue, M., Kameno, S., \& Nagai, H. 2008a, ApJ, 675, 79

Asada, K., Inoue, M., Nakamura, M., Kameno, S., \& Nagai, H. 2008b, ApJ, 682,798

Asada, K., Inoue, M., Uchida, Y., et al. 2002, PASJ, 54, L39

Asada, K., Nakamura, M., Inoue, M., Kameno, S., \& Nagai, H. 2010, ApJ, 720, 41

Attridge, J. M., Wardle, J. F. C., \& Homan, D. C. 2005, ApJ, 633, 85

Blandford, R. 1993, in Astrophysical Jets, ed. M. Burgarella, M. Livio, \& C. P. O'Dea (Astrophysics and Space Science Library, Vol. 103; Cambridge: Cambridge Univ. Press), 15

Blandford, R. D., \& Znajek, R. L. 1977, MNRAS, 179, 433

Broderick, A. E., \& McKinney, J. C. 2010, ApJ, 725, 750

Burn, B. J. 1966, MNRAS, 133, 67

Chen, T. 2005, PhD thesis, Brandeis Univ.

Clausen-Brown, E., Lyutikov, M., \& Kharb, P. 2011, MNRAS, 415, 2081

Contopoulos, I., Christodoulou, D. M., Kazanas, D., \& Gabuzda, D. C. 2009, ApJ, 702, L148

Croke, S., \& Gabuzda, D. C. 2008, MNRAS, 386, 619

Croke, S. M., O'Sullivan, S. P., \& Gabuzda, D. C. 2010, MNRAS, 402, 259

Gabuzda, D. C., Murray, E., \& Cronin, P. 2004, MNRAS, 351, L89

Gómez, J.-L., Marscher, A. P., Alberdi, A., Jorstad, S. G., \& Agudo, I. 2002, VLBA Scientific Memo No. 30

Gómez, J.-L., Marscher, A. P., Jorstad, S. G., Agudo, I., \& Roca-Sogorb, M. 2008, ApJ, 681, L69

Gómez, J.-L., Roca-Sogorb, M., Agudo, I., Marscher, A. P., \& Jorstad, S. G. 2011, ApJ, 733, 11

Homan, D. C. 2012, ApJ, 747, L24

Homan, D. C., Lister, M. L., Aller, H. D., Aller, M. F., \& Wardle, J. F. C. 2009, ApJ, 696, 328

Hovatta, T., Valtaoja, E., Tornikoski, M., \& Lähteenmäki, A. 2009, A\&A, 494, 527

Hughes, P. A., Aller, H. D., \& Aller, M. F. 1989, ApJ, 341, 68
Kellermann, K. I., Lister, M. L., Homan, D. C., et al. 2004, ApJ, 609, 539 Komatsu, E., Dunkley, J., Nolta, M. R., et al. 2009, ApJS, 180, 330

Kovalev, Y. Y., Lobanov, A. P., Pushkarev, A. B., \& Zensus, J. A. 2008, A\&A, 483,759

Laing, R. 1980, MNRAS, 193, 439

Lister, M. L., Aller, H. D., Aller, M. F., et al. 2009a, AJ, 137, 3718

Lister, M. L., Cohen, M. H., Homan, D. C., et al. 2009b, AJ, 138, 1874

Lister, M. L., \& Homan, D. C. 2005, AJ, 130, 1389

Lister, M. L., Tingay, S. J., Murphy, D. W., et al. 2001, ApJ, 554, 948

Lobanov, A. P. 1998, A\&A, 330, 79

Lyutikov, M., Pariev, V. I., \& Gabuzda, D. C. 2005, MNRAS, 360, 869

Mahmud, M., Gabuzda, D., \& Bezrukovs, V. 2009, MNRAS, 400, 2

Mantovani, F., Rossetti, A., Junor, W., Saikia, D. J., \& Salter, C. J. 2010, A\&A, 518, A33

Marr, J. M., Taylor, G. B., \& Crawford, F., III 2001, ApJ, 550, 160

Marscher, A. P., Jorstad, S. G., D'Arcangelo, F. D., et al. 2008, Nature, 452, 966

McKinney, J. C., \& Narayan, R. 2007, MNRAS, 375, 531

Meier, D. L., Koide, S., \& Uchida, Y. 2001, Science, 291, 84

Murphy, E., \& Gabuzda, D. C. 2012, J. Phys. Conf. Ser., 355, 012009

Mutel, R. L., Denn, G. R., \& Dreier, C. 2005, in ASP Conf. Proc. 340, Future Directions in High Resolution Astronomy: The 10th Anniversary of the VLBA, ed. J. Romney \& M. Reid (San Francisco, CA: ASP), 155

O’Sullivan, S. P., \& Gabuzda, D. C. 2009, MNRAS, 393, 429

Pacholczyk, A. G. 1970, Radio Astophysics, Nonthermal Processes in Galactic and Extragalactic Sources (San Francisco, CA: Freeman)

Press, W. H., Flannery, B. P., Teukolsky, S. A., \& Vetterling, W. T. 1992, Numerical Recipes in FORTRAN 77 (2nd ed.; Cambridge: Cambridge Univ. Press)

Pushkarev, A. B. 2001, Astron. Rep, 45, 667

Pushkarev, A. B., Hovatta, T., Kovalev, Y. Y., et al. 2012, A\&A, in press (arXiv:1207.5457)

Reichstein, A., \& Gabuzda, D. 2011, in Proc. 10th European VLBI Network Symp. and EVN Users Meeting VLBI and the New Generation of Radio Arrays (PoS; Trieste: SISSA), 044

Reichstein, A., \& Gabuzda, D. 2012, J. Phys.: Conf. Ser., 355, 012021

Roberts, D. H., Wardle, J. F. C., \& Brown, L. F. 1994, ApJ, 427, 718

Rudnick, L., \& Jones, T. W. 1983, AJ, 88, 518

Rusk, R. E. 1988, Ph.D. thesis, Univ. Toronto

Savolainen, T., Wiik, K., Valtaoja, E., \& Tornikoski, M. 2008, in ASP Conf. Ser. 386, Extragalactic Jets: Theory and Observation from Radio to Gamma Ray, ed. T. A. Rector \& D. S. De Young (San Francisco, CA: ASP), 451

Shepherd, M. C. 1997, in ASP Conf. Ser. 125, Astronomical Data Analysis Software and Systems VI, ed. G. Hunt \& H. E. Payne (San Francisco, CA: ASP), 77

Sokolovsky, K. V., Kovalev, Y. Y., Pushkarev, A. B., \& Lobanov, A. P. 2011, A\&A, 532, 38

Taylor, G. B. 1998, ApJ, 506, 637 
Taylor, G. B. 2000, ApJ, 533, 95

Taylor, A. R., Stil, J. M., \& Sunstrum, C. 2009, ApJ, 702, 1230

Taylor, G. B., \& Zavala, R. T. 2010, ApJ, 722, L183

Tribble, P. C. 1991, MNRAS, 250, 726

Udomprasert, P. S., Taylor, G. B., Pearson, T. J., \& Roberts, D. H. 1997, ApJ, 483, L9

Vlahakis, N., \& Königl, A. 2004, ApJ, 605, 656
Walker, R. C., Dhawan, V., Romney, J. D., Kellermann, K. I., \& Vermeulen, R. C. 2000, ApJ, 530, 233

Wrobel, J. M. 1993, AJ, 106, 444

Zavala, R. T., \& Taylor, G. B. 2002, ApJ, 566, L9

Zavala, R. T., \& Taylor, G. B. 2003, ApJ, 589, 126

Zavala, R. T., \& Taylor, G. B. 2004, ApJ, 612, 749

Zavala, R. T., \& Taylor, G. B. 2005, ApJ, 626, L73 